\title{
Disc accretion onto white dwarfs
}

\author{
Dissertation \\ zur Erlangung des Doktorgrades \\ der Mathematisch-Naturwissenschaftlichen Fakultäten \\ der Georg August-Universität zu Göttingen
}

vorgelegt von

Matthias Schreiber

aus Göttingen 
D 7

Referent: PD Dr. K. Mannheim

Korreferent: Prof. Dr. U. Christensen

Tag der mündlichen Prüfung: 29.01.2001 


\section{Abstract: Disc accretion onto white dwarfs}

In non-magnetic cataclysmic variables (CVs) a white dwarf accretes matter from a mainsequence secondary star via an accretion disc. The dynamical behaviour of the accretion disc determines the accretion rate onto the white dwarf. Thermal instabilities in the accretion disc associated with the ionisation of hydrogen can lead to a limit-cycle behaviour in which the disc switches quasi-periodically between high and low accretion states. This thermal limit-cycle model is the generally accepted explanation for dwarf nova outbursts observed in many CVs. The process of disc accretion in non-magnetic CVs is subject to a number of external conditions, namely mass transfer variations of the secondary star, stream overflow and irradiation by the white dwarf. In this thesis I develop a model for time-dependent disc accretion onto white dwarfs and analyse the influence of these external conditions on the accretion process.

I examine the effects of mass transfer variations by deriving real mass transfer variations from light curve monitoring of the disc-less CV AM Her. These mass transfer variations I include in simulations of disc accretion onto white dwarfs in non-magnetic systems and find that the mass accretion rate of the disc relaxes to an equilibrium with the prevailing mass transfer rate on a rather short timescale. I conclude that the observed changes in outburst duration and outburst magnitude are caused by nearly simultaneous variations of the mass loss rate from the secondary.

I also present a new model for the stripping of the stream by the accretion disc, and find that stream overflow can have subtle effects on the evolution of the accretion disc only if the amount of overflowing stream material exceeds $25 \%$ of the mass transfer rate. I conclude that solely very large stream overflow fractions can change the outbursts of dwarf novae. For realistic amounts of stream overflow the overall outburst behaviour is marginally changed.

The accretion disc is mostly influenced by the white dwarf irradiation. I present a selfconsistent model for irradiated accretion discs and find that efficient irradiation in dwarf nova systems causes small "echo" outbursts following the larger ones immediately. This result contrasts with the observations of dwarf nova outbursts. As an explanation for this discrepancy I suggest that the reprocessing efficiency of disc irradiation is rather small. This is in agreement with results I obtain from detailed simulations of irradiated discs in post novae. These systems are excellent laboratories for studying the effects of disc irradiation because the white dwarf heated during the nova eruption provides a much stronger irradiation of the disc than in normal dwarf novae. I derive time-limits for the occurrence of dwarf nova outbursts in post novae and present detailed simulations of the evolution of irradiated discs in post novae.

In addition to the developed theory of irradiated discs around white dwarfs, I show preliminary results of an intensive observing campaign on the post nova system V446 Her. Finally, discussing the influence of disc irradiation on the post nova evolution in the light of the current working hypothesis leads me to put forward a new scenario. 



\section{Contents}

1 Introduction 1

2 Cataclysmic Variables: a brief overview 5

2.1 Roche geometry and mass transfer . . . . . . . . . . 5

2.2 The zoo of CVs . . . . . . . . . . . . . . . 7

2.3 Nova eruptions . . . . . . . . . . . . . . . 8

2.4 The evolution of CVs . . . . . . . . . . . . . . . . 9

3 Theory of accretion discs $\quad 15$

3.1 Thin discs . . . . . . . . . . . . . . . . 15

3.2 Shapiro-Lightman-Eardly solution . . . . . . . . . . . 16

3.3 Advection dominated accretion flows . . . . . . . . . . . . . 16

3.4 Thermal equilibrium and stability . . . . . . . . . . . . 19

4 Dwarf nova outbursts and disc instabilities 21

4.1 Assumptions and time scales . . . . . . . . . . . . 21

4.2 Vertical structure . . . . . . . . . . . . . . . . . . . 24

4.2.1 The equations . . . . . . . . . . . . . . . 24

4.2.2 The S-curve in the $\Sigma-T_{\text {eff }}$ diagram . . . . . . . . 26

4.2.3 Instabilities and the thermal limit-cycle . . . . . . . . 30

4.3 The vertically averaged description . . . . . . . . . . . 32

4.4 Simulation of dwarf nova outbursts . . . . . . . . . . . . 33

4.5 Confrontation with observation . . . . . . . . . . . . 38

5 AM Her as a dwarf nova 41 
5.1 The cause of mass transfer variations . . . . . . . . . . . . . 42

5.2 The mass loss rate of the secondary star in AM Herculis . . . . . . 43

5.3 Results . . . . . . . . . . . . . . . . . . . 45

5.3.1 The fictitious dwarf nova . . . . . . . . . . 45

5.3.2 The mass transfer and mass accretion rates . . . . . . 48

5.3.3 Dependence on the primary mass . . . . . . . . . . . 49

5.4 Discussion and conclusions . . . . . . . . . . . . . 53

6 Stream overflow and dwarf nova outbursts 55

6.1 The equations . . . . . . . . . . . . . . . 56

6.2 Stripping of the stream . . . . . . . . . . . 58

6.3 Results . . . . . . . . . . . . . . . . . . . . 60

6.4 Conclusions . . . . . . . . . . . . . . . . 63

7 Irradiated accretion discs around white dwarfs $\quad 65$

7.1 Vertical structure . . . . . . . . . . . . . . . 66

7.2 Dwarf nova outbursts of irradiated accretion discs . . . . . . . 72

7.3 Dwarf novae among post novae . . . . . . . . . . . . 76

7.3.1 White dwarf cooling in post novae . . . . . . . . 77

7.3.2 Time-dependent disc irradiation . . . . . . . . 78

7.3.3 Irradiation limits on the occurrence of dwarf novae in post novae ....................... 78

7.3.4 Detailed long term light curves of post novae . . . . . 80

7.4 Conclusion . . . . . . . . . . . . . . . . . . 84

8 Future targets: the fortunate case of V $446 \mathrm{Her} \quad 87$

8.1 Observations ..................... 87

8.2 Proposed observations: detecting the hottest white dwarf in a dwarf nova . . . . . . . . . . . . 88

9 Implications: a new post nova scenario?

9.1 The hibernation scenario for post novae . . . . . . . . . . . . 91

9.2 The visual decline in post novae and disc irradiation . . . . . . . . 92 
9.3 Mass transfer cycles instead of hibernation? . . . . . . . . . . . . 94

$\begin{array}{ll}10 \text { Summary } & 97\end{array}$

$\begin{array}{ll}\text { Bibliography } & 101\end{array}$

$\begin{array}{ll}\text { Acknowledgement } & 109\end{array}$

$\begin{array}{ll}\text { List of publications } & 111\end{array}$

$\begin{array}{ll}\text { Curriculum Vitae } & 113\end{array}$ 



\section{Chapter 1}

\section{Introduction}

Accretion, the infall of matter onto a more or less compact object under the action of gravitation, is of particular importance in astrophysics, because gravitation dominates the three other fundamental interactions on macroscopic scales. The gravitational energy released by accretion with a rate $\dot{M}_{\text {acc }}$ onto the surface of a body of mass $M$ and radius $R$ is

$$
L_{\mathrm{acc}}=\frac{G M \dot{M}_{\mathrm{acc}}}{R}
$$

where $\mathrm{G}$ is the gravitational constant. The smaller the accreting object and the larger its mass, the more energy is released for a given accretion rate. The conversion of mass into energy due to accretion onto compact objects determines the emission properties of a wide variety of astrophysical objects ranging from the galactic to the planetary scale. Indeed, the presence of accretion flows is established around protostars at the beginning of the stellar evolution as well as around accreting white dwarfs, neutron stars, and black holes, where the accreting object is a compact star that reached the end of its stellar evolution.

In active galaxies the emission from a central non-stellar source is immense, and in some of these active galactic nuclei (AGN) the central engine generates more energy than all the stars in the host galaxy. It is now widely accepted, that these central engines are powered by accreting supermassive black holes, as it was conjectured first by Salpeter (1964). In this picture energy is generated by gravitational infall onto the massive black hole $\left(10^{6}-10^{9} M_{\odot}\right)$ via a dissipative accretion disc. The acceptance of this idea results from the fact that accretion onto a black hole is a very efficient process for converting mass into energy and, hence, can explain the huge observed luminosities. In addition, superluminal jets are seen in some AGN, suggesting a relativistic source for the jet (e.g. Blandford 1992). Moreover, during recent years spectroscopic studies of the centres of nearby galaxies have 
indicated that most active (and even some non-active) galaxies contain massive black holes. Indeed there is some strong evidence for the presence of a supermassive black hole in the centre of our own galaxy (Genzel et al. 1997). Although the details of the accretion process in these systems are yet unclear, the results mentioned above suggest that accretion onto supermassive black holes is of particular importance in the formation and evolution of galaxies.

In the star formation process too accretion is the focus of attention. Stars are formed by the collapse of rotating molecular clouds. As a result of angular momentum conservation, a flat disc forms with the protostar in the centre. The protostar begins to evolve into a main sequence star and accretes material from the surrounding disc. Many indirect signs for such accretion discs being present in young stellar objects (YSOs) have been found over the last decades. The spectral energy distribution of T Tauri stars is in general explained by considering the contribution of a thin accretion disc. Moreover, FU Ori objects show states in which the emission of the accretion disc is believed to exceed the contribution of the central protostar (Beckwith 1994). More recently, observations have shown direct evidence for discs around YSOs. The first and most famous case is the dusty disc around $\beta$ Pictoris observed by Smith \& Terrile (1984). In the meantime observations with the Hubble Space Telescope (HST) have shown that such discs are common (e.g. Malbet et al. 1993). This is interesting because accretion in YSOs is related to the process of planet formation. Indeed, there is evidence that not only discs around protostars but also planets around main sequence stars are quite common (Marcy et al. 1999).

The third and, in the context of this thesis, most important group of objects with significant accretion are the interacting binary stars. In these systems a compact object accretes material from the Roche lobe filling secondary. In Cataclysmic Variables (CVs) the compact object is a white dwarf which accretes mass from a low mass, late-type secondary. In $X$-Ray binaries (XBs) the compact object is a neutron star or a black hole. Interacting binaries represent excellent laboratories to study the physics of accretion. Compared with other accreting objects the dynamical timescale on which accretion proceeds in these binaries is relatively short, and, therefore, the variability connected with accretion can be studied within an average scientist's lifetime. Another advantage results from the fact that the conditions under which accretion occurs are best observable in interacting binaries, as it is rather easy possible to measure the orbital period and the stellar masses (which, together, determine the disc size), the inclination, and the mass transfer rate. Compact binary systems, and specifically $\mathrm{CVs}$, therefore provide a supreme astrophysical environment for detailed studies of accretion discs in general.

CVs appear in various different subspecies, according to their size, to the strength of the magnetic field on the white dwarf and to the amount of mass transfer. 
Therefore a large variety of physical processes is involved in the phenomenon of accretion onto white dwarfs in CVs. Of major importance in the context of disc accretion onto white dwarfs is the thermal limit-cycle model (e.g. Cannizzo 1993b). It is regarded as the accretion disc model which has linked the theory of accretion discs to observations most successfully. Although developed in order to explain sudden brightenings called dwarf nova outbursts which are observed in some CVs, the thermal limit cycle model is also applicable to YSOs and AGN.

In spite of its success, the common version of the thermal limit-cycle model cannot be considered as fully satisfactory. From the theorist's point of view the model should be improved as it completely ignores the influence which the environment of the accretion disc in a CV has on its structure and its dynamics. The most important effects are: (1) the irradiating flux emitted by the white dwarf; (2) the form of mass addition to the accretion disc from the accretion stream; (3) the time-dependence of mass transfer determined by the dynamics of the secondary. The comparison of the results obtained from the thermal limit-cycle model with observations leads to strong arguments which provide support for this model but yet no detailed calculation exists that achieves a satisfying, quantitative agreement with hard observational facts for a given system. Thus, further refinement of the theory is required.

In the following I discuss the conditions under which disc accretion occurs in CVs. In chapter 2 and 3 I give an overview of CVs and the theory of accretion flows, the topics which are linked together in this thesis. Thereafter I explain the thermal limit-cycle model, present my version of the model and compare it with other calculations. Having developed a state-of-the-art model, I then analyse the influence of the environment in a $\mathrm{CV}$ on the accretion process. This improves our understanding of $\mathrm{CVs}$ as well as it provides deep insights into the theory of accretion discs with important applications to astrophysics in general. Finally, I discuss my results in the context of the $\mathrm{CV}$ evolution. 


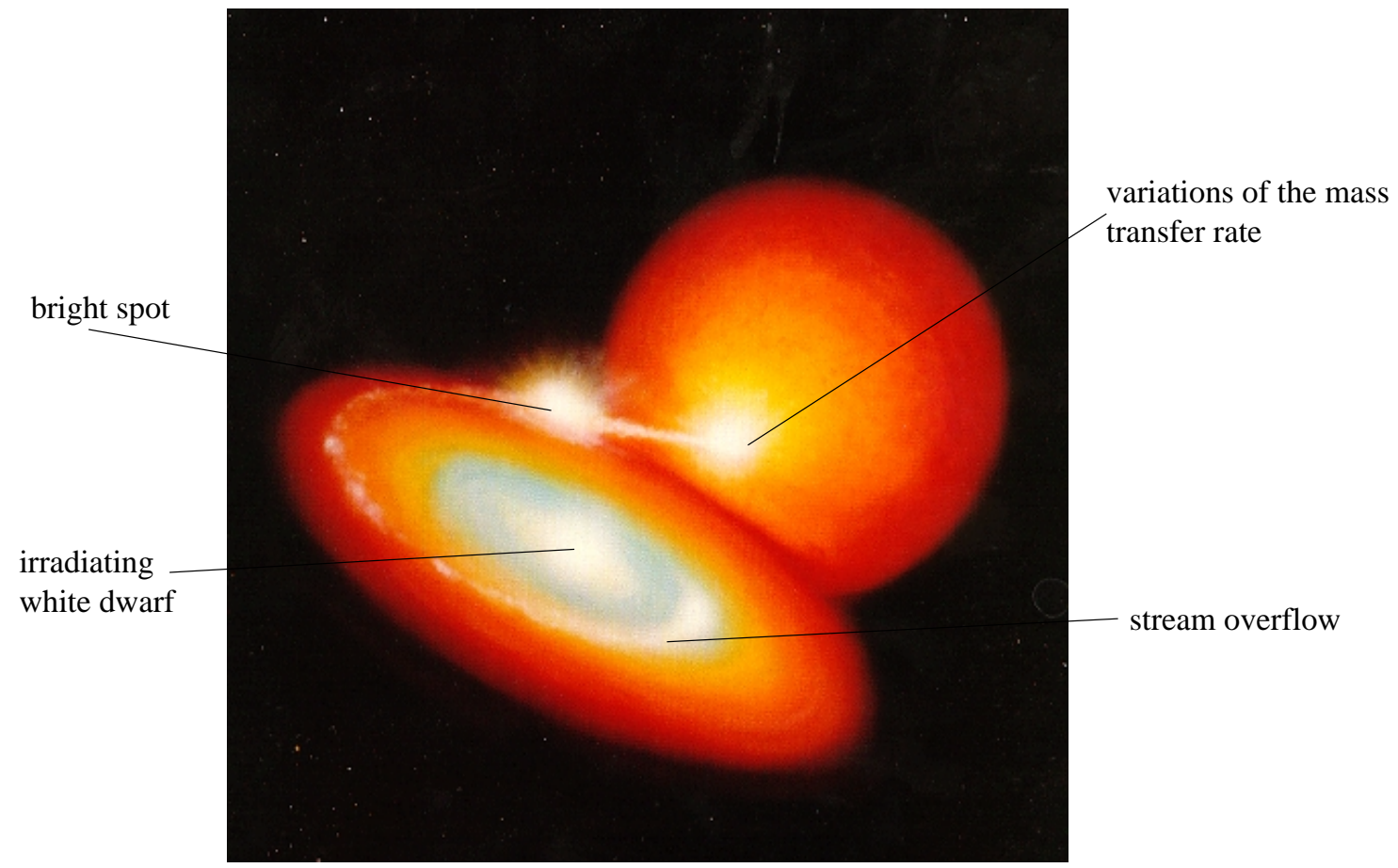

Figure 1.1: Schematic picture of a non-magnetic cataclysmic variable. The companion of the white dwarf, a low mass main sequence star, transfers mass to the accretion disc around the white dwarf. A bright spot is formed at the impact of the stream on the accretion disc. 


\section{Chapter 2}

\section{Cataclysmic Variables: a brief overview}

This chapter gives an overview of the class of Cataclysmic Variables (CVs), a major class of systems in which disc accretion occurs onto white dwarfs. I only briefly review the geometry and phenomenology of CVs whereas I provide a more detailed discussion of nova eruptions and the evolution of CVs as the results of this thesis will change the common picture of these processes.

\subsection{Roche geometry and mass transfer}

The basic kinematics of close binary systems and, hence, of CVs are well described using Kepler's third law in the generalised Newtonian formulation,

$$
a^{3}=\frac{G}{4 \pi^{2}}\left(M_{\mathrm{wd}}+M_{\mathrm{sec}}\right) P_{\mathrm{orb}}^{2}
$$

relating the binary separation $a$ to the orbital period $P_{\text {orb }}$ and the sum of primary mass $\left(M_{\mathrm{wd}}\right)$ and secondary mass $\left(M_{\mathrm{sec}}\right)$. The range of known orbital periods of CVs covers 18 min- $2 \mathrm{~d}$. For the shorter orbital periods the binary separation is similar to the distance between earth and moon $\left(\sim 4 \times 10^{10} \mathrm{~cm}\right)$. In other words, the sun $\left(R_{\odot} \sim 7 \times 10^{10} \mathrm{~cm}\right)$ is large enough to contain a classical CV. Hence, CVs are rather small stars but, as we will see, they contain big physics.

The gravitational potential of a binary system, written in a frame of reference rotating with the binary system,

$$
\Phi(\vec{R})=-\frac{G M_{\mathrm{wd}}}{\left|\vec{R}-\vec{R}_{2}\right|}-\frac{G M_{\mathrm{sec}}}{\left|\vec{R}-\vec{R}_{1}\right|}-\frac{1}{2}(\vec{\omega} \times \vec{R})^{2},
$$




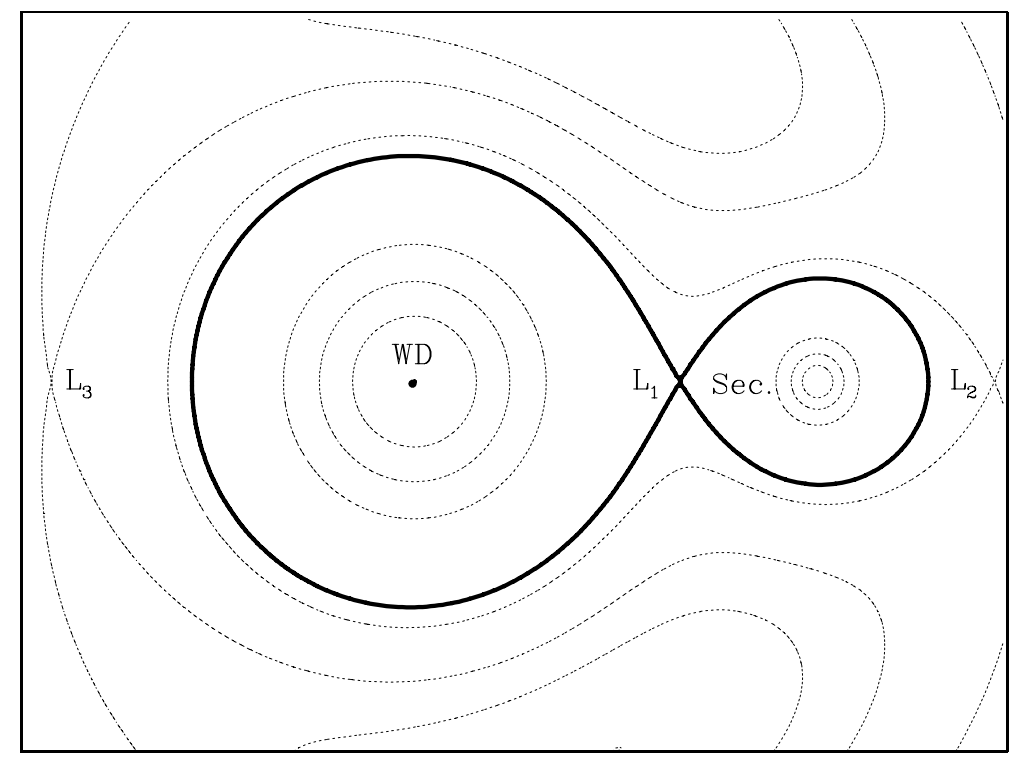

Figure 2.1: Equipotential surfaces in a CV with $M_{\mathrm{wd}} / M_{\mathrm{sec}}=5$. The thick line displays the critical Roche surface. In case the secondary (Sec.) fills its Roche lobe, mass is transferred through the $L_{1}$ point into the gravitational well of the white dwarf (WD).

defines critical equipotential surfaces which limit the radial extend of the components. In Eq (2.2) $\vec{R}_{1}$ and $\vec{R}_{2}$ are the position vectors of the two stars approximated by point masses, $\vec{\omega}$ is the angular velocity of the binary, and $\vec{R}$ is the radius vector of the centre of mass. The Roche lobes of the two stars are in contact at the inner Lagrangian point $L_{1}$ which is a saddle point of $\Phi(\vec{R})$. Fig. 2.1 shows the equipotential surfaces in a CV with $M_{\mathrm{wd}} / M_{\mathrm{sec}}=5$.

In case the secondary fills its Roche lobe, mass is transferred through the $L_{1}$ point into the Roche lobe of the primary. Detailed studies of stream lines in the vicinity of the $L_{1}$ point and Roche lobe overflow are given by Lubow \& Shu $(1975,1976)$. After leaving $L_{1}$, the stream particles fall toward the white dwarf with increasing velocity. What happens next strongly depends on the strength of the magnetic field of the primary: (a) in the so called non-magnetic systems $(B<0.1 \mathrm{MG})$, the stream leaving the $L_{1}$ point cannot hit the white dwarf directly (because the angular momentum is conserved) but is slung back by the gravity of the white dwarf. As for a given angular momentum a circular orbit has the least energy the stream tends to form a ring which will become a disc due to further mass transfer and angular momentum transport by viscous processes; (b) in the magnetic polars $(B>10 \mathrm{MG})$ (Buckley \& Warner 1995) the infalling matter couples onto the strong magnetic field of the white dwarf before it can build a disc and is funnelled to accretion region(s) near the magnetic pole(s) of the white dwarf; (c) in inter- 
mediate polars, i.e. CVs containing a weakly magnetic white dwarf, a partial disc may exist with the mass flowing from the inner edge of the disrupted disc through magnetically funnelled accretion curtains onto the white dwarf.

\subsection{The zoo of CVs}

This section gives a short overview on observational characteristics of CVs and the resulting classification scheme.

Apart from the three broad subclasses, defined at the end of the previous section, there exist various subtypes of CVs referring to differences in the observed phenomenology:

- Dwarf novae are non-magnetic systems in which the white dwarf accretes material from a Roche lobe-filling late type secondary star via an accretion disc. The characteristic brightenings with amplitudes of 2-8 magnitudes at visual wavelength known as dwarf nova outbursts typically last a few days to weeks and recur quasi-periodically on timescales of weeks to years. There are three subtypes of dwarf novae characterised by the morphology of their outbursts. ZCam stars show in addition to dwarf nova outbursts standstills which continue for up to $\sim 80$ days. SU Uma stars show so-called superoutbursts which are roughly $1 \mathrm{mag}$ brighter and last 5 times longer than normal outbursts. Almost all of these systems have rather short orbital periods $(<2 \mathrm{hr})$. The third subclass, called the $U$ Gem class, contains all the dwarf novae that are neither Z Cam nor SU Uma stars.

- Classical novae have only one observed major brightening of 6-19 mag called nova eruption. It is common to distinguish between fast novae lasting only a few weeks and having a larger amplitude and slow novae with low amplitudes in eruption but a duration of years.

- Recurrent novae are classical novae which were found to repeat their eruptions. To distinguish between recurrent and dwarf novae it is necessary to consider their spectroscopic characteristics because in recurrent (and classical) novae a substantial shell is ejected. This difference is, as we will see, connected with the completely different physical origins of dwarf nova outbursts and recurrent novae.

- Nova-like variables represent the subclass of non-eruptive CVs. This definition does not exclude the possibility that nova-like variables are also members of other classes like classical novae but without an observed eruption. 
Notice, as in the case of nova-likes, an individual CV can be a member of more than one class, e.g. when dwarf nova outbursts are observed after a nova eruption. It is also interesting to realise that nova eruptions and dwarf nova outbursts resemble each other in some cases and, hence, a number of large amplitude/low outburst frequency dwarf novae are found in the lists of nova remnants, the most famous example being the short-period dwarf nova WZ Sge (Duerbeck 1987).

The two types of sudden brightenings shown by $\mathrm{CVs}$, i.e. dwarf nova outbursts and nova eruptions, have completely different physical origins: dwarf nova outbursts are thought to result from thermal instabilities associated with hydrogen ionisation in an accretion disc (see Cannizzo 1993b for a review, Ludwig et al. 1994 for a detailed parameter study, and chapter 4 of this thesis); a nova eruption arises when hydrogen-rich material, accreted onto the surface of a white dwarf, ignites under degenerate conditions (see Starrfield et al. 1998 for a review).

As the dynamical behaviour of accretion discs around white dwarfs, which is the main subject of this thesis, may be affected by nova eruptions on the surface of the white dwarf, I give a short review of the theory of nova eruptions in the following section.

\subsection{Nova eruptions}

The initial conditions under which a nova eruption can arise on the surface of the white dwarf were first examined by Giannone \& Weigert (1967) and more recently by Prialnik (1986) and Starrfield et al. $(1985,1986)$. The nova process is strongly connected to the equation of state for degenerate matter,

$$
P \propto \rho^{\gamma}
$$

where $P$ is the pressure, $\rho$ denotes the density, and $\gamma$ is $5 / 3$ in the non-relativistic case and 4/3 in the relativistic case, respectively. The pressure in Eq. (2.3) is independent of the temperature. In case the temperature and the density of the accreted envelope reach values sufficient for nuclear reactions, any small increase in temperature, leads to enhanced energy generation (but not to an increase in pressure). This causes runaway heating until the temperature exceeds the Fermi temperature, where the equation of state switches to that of a perfect gas and the envelope can cool via expansion. Whether the expansion exceeds the escape velocity depends on the pressure at the base of the envelope $P_{\mathrm{b}}$. MacDonald (1983) shows that

$$
P_{\mathrm{b}}=\frac{G M_{\mathrm{wd}}}{R_{\mathrm{wd}}^{2}} \frac{M_{\mathrm{e}}}{4 \pi R_{\mathrm{wd}}^{2}}>10^{20} \mathrm{dyn}
$$


is sufficient for the envelope being ejected. In Eq. (2.4) $M_{\mathrm{wd}}$ is the mass of the white dwarf, $G$ the gravitational constant and $M_{\mathrm{e}}$ the mass of the envelope. As the envelope mass which is necessary to ignite the nuclear burning decreases with increasing white dwarf mass, and since the radius of white dwarfs is inversely proportional to its mass, the critical envelope mass decreases with increasing white dwarf masses.

The nuclear reactions which heat up the envelope are at first the proton-proton chain until the temperature has reached $T \sim 10^{7} \mathrm{~K}$ and CNO reactions become dominant. As in the cores of high mass stars, the temperature sensitivity of the energy generation by this process leads to the onset of convection, and at $T \sim 8 \times 10^{7} \mathrm{~K}$ the envelope is fully convective. For $T>10^{8} \mathrm{~K}$, the lifetimes of the $\beta^{+}$unstable nuclei $\left({ }^{13} \mathrm{~N},{ }^{14} \mathrm{O},{ }^{15} \mathrm{O},{ }^{17} \mathrm{~F}\right)$ exceed the time scale of proton capture so that they become the most abundant nuclei. The lifetime of the $\beta^{+}$unstable nuclei is of the order of the convection turn-over time ( $\sim 100 \mathrm{~s})$ and therefore convection deposits energy at the surface of the envelope as well as it brings "fresh" unprocessed CNO nuclei into the high temperature zone. Finally, at temperatures of $\sim 2.5 \times 10^{9} \mathrm{~K}$ the envelope expands and nuclear burning is terminated (Bode \& Evans 1989).

After the nuclear burning turned off, the system becomes a normal CV, but, as the white dwarf is heated up to $\sim 3 \times 10^{5} \mathrm{~K}$ during the nuclear burning period, the nova event has deep implications for the accretion disc in the post nova system: the slowly cooling white dwarf is intensively irradiating the accretion disc. This irradiation has a dramatic impact on the disc's structure which is examined in chapter 7.

\subsection{The evolution of CVs}

Thus far I have described various phenomena observed in CVs without mentioning where these systems came from, how they live, or where they go. Before I will go into the details of disc accretion onto white dwarfs I want to briefly review the standard scenario of cataclysmic variable evolution in order to be able to discuss the connections between disc accretion and $\mathrm{CV}$ evolution later.

A CV is born from a wide binary with a long orbital period consisting of a low mass main-sequence star and a more massive primary. The initial mass of the primary lies in the range of $1<M_{1}<10$, because a lower mass has not yet had the time to evolve and a higher mass leads to the formation of a neutron star. The primary evolves on its nuclear timescale $\left(10^{6}-10^{10} \mathrm{yr}\right)$ into a giant with a dramatically expanded radius. The radius $R_{1}$ depends on the mass $M_{\mathrm{c}}$ of the 
degenerate carbon-oxygen core (Ritter 1976):

$$
\log R_{1}=1.48+1.3 M_{\mathrm{c}} / M_{\odot} .
$$

The primary fills its Roche lobe and mass transfer from the primary to the secondary starts. This mass transfer is not reduced by the mass loss, as the radius of the primary's envelope does not depend on its mass (the radius of the envelope depends only on the core mass $M_{\mathrm{c}}$ (Eq. (2.5)). As a result, a runaway mass transfer of up to $0.1 M_{\odot} \mathrm{yr}^{-1}$ occurs. Due to the fact that mass is transferred from the more massive to the less massive component, the binary separation shrinks. As the high mass transfer rate exceeds the rate permitted by the Eddington-limit, the transferred mass fills the outer Roche lobe of the secondary and then grows to form an extended common envelope around the stellar components. During this period the binary loses angular momentum due to frictional braking, which further reduces the binary separation. This can either lead to the birth of a pre-CV or to coalescence of the system, depending on the density gradient outside the degenerate core (Hjellming \& Taam 1991; Taam \& Bodenheimer 1991).

However, after the ejection of the envelope of the primary exposing the detached system, the binary system has to lose angular momentum or the secondary has to expand in order to develop into a semi-detached configuration. As the secondary evolves on the main sequence to larger radii one might believe that expansion of the secondary can drive the mass transfer. But, it is evident that the system has to lose angular momentum to start and proceed mass transfer, because the timescale on which low mass stars evolve on the main sequence is longer than the Hubble time. The loss of angular momentum in this pre-CV stage may be driven by emission of gravitational radiation or by magnetic stellar wind braking. For the large orbital periods of the detached binary after the common envelope period, magnetic braking is the dominant process.

Once mass transfer has started and the $\mathrm{CV}$ is born, it is important to examine the stability of this mass transfer. In order to do so one has to compare the change in radius of the secondary (due to adjustment of its internal structure in response to mass loss) with the change of its Roche lobe radius due to orbital changes (induced by the shift of mass within the binary). In general, the smaller the mass ratio $q \equiv \frac{M_{\mathrm{sec}}}{M_{\mathrm{wd}}}$ the larger the expansion of the Roche lobe of the secondary for a given amount of mass loss. Thus, small mass ratios tend to stabilise mass transfer and there exists a critical mass ratio above which mass transfer becomes unstable. In Warner (1995) this critical mass ratio is given as $q_{\text {crit }} \sim 1.26$. More detailed studies on this subject are presented by de Kool (1992). The stability criterion explains why only low mass $\mathrm{K}$ - and $\mathrm{M}$-stars are found as secondaries in CVs.

As noted above, the main mechanisms for loosing angular momentum are magnetic braking and gravitational radiation. After mass transfer started, an additional 
angular momentum loss due to nova eruptions occurs. A nova eruption creates a temporary common envelope during which friction transmits orbital momentum from the secondary to the envelope, which is then expelled from the system. This is in addition to the angular momentum carried away by the ejecta. Schenker et al. (1998) found that the additional angular momentum loss due to nova eruptions causes discontinuous mass transfer during the evolution, but that it is to low to drive the evolution alone. Gravitational radiation is also inefficient for systems with long orbital periods and thus angular momentum loss by a wind magnetically linked to the secondary which is called magnetic braking is the currently favoured option for systems with longer orbital periods. The relative paucity of CVs in the period range of $2-3 \mathrm{hr}$, called the period gap, can be explained in this picture due to disrupted magnetic braking when the secondary becomes fully convective. This occurs when the mass of the secondary is around $0.2 M_{\odot}$ which coincides with orbital periods around $3 \mathrm{hr}$. The basic idea of the disrupted magnetic braking model is that the change of the internal strucure of the secondary from a deep convective envelope to total convection leads to a rearrangement of its magnetic field structure. The resulting sudden decrease of the mass transfer rate causes the secondary to shrink below its Roche lobe.

Without magnetic braking and without mass transfer, the binary then evolves more slowly to lower orbital periods because the only mechanism to lose angular momentum is gravitational radiation which acts on a longer timescale than magnetic braking:

$$
\begin{gathered}
\tau_{\mathrm{GR}}=3.8 \times 10^{11} \frac{\left(M_{\mathrm{wd}}+M_{\mathrm{sec}}\right)^{1 / 3}}{M_{\mathrm{wd}} M_{\mathrm{sec}}} P_{\mathrm{orb}}^{8 / 3}(\mathrm{~d}) \mathrm{yr}, \\
\tau_{\mathrm{MB}}=2.2 \times 10^{9} \frac{M_{\mathrm{wd}}}{\left(M_{\mathrm{wd}}+M_{\mathrm{sec}}\right)^{1 / 3}} R_{\mathrm{sec}}^{-4} P_{\mathrm{orb}}^{10 / 3}(\mathrm{~d}) \mathrm{yr},
\end{gathered}
$$

where $M_{\mathrm{wd}}$ is the white dwarf mass, $M_{\mathrm{sec}}$ the mass of the secondary, $P_{\mathrm{orb}}(\mathrm{d})$ the orbital period in days and $R_{\mathrm{sec}}$ is the radius of the secondary in solar radii (Kolb $\&$ Stehle 1996). After $\sim 10^{9} \mathrm{yr}$ and at orbital periods of $\sim 2 \mathrm{hr}$ the binary comes into contact again, i.e. the secondary fills its Roche lobe and mass transfer starts. It takes further $\sim 10^{9} \mathrm{yr}$ until the secondary becomes a brown dwarf and the $\mathrm{CV}$ reaches its minimum orbital period of $\sim 80 \mathrm{~min}^{1}$.

Fig. 2.2 shows an evolutionary track for the mass transfer rate in CVs as a function of orbital period following the standard scenario. I used the expressions obtained by McDermott \& Taam (1989) for mass transfer due to magnetic braking above the period gap

$$
\dot{M}_{\text {tr }}=2.00 \times 10^{-11} P_{\text {orb }}^{3.7}(\mathrm{hr}) M_{\odot} \mathrm{yr}^{-1},
$$

\footnotetext{
${ }^{1}$ Note that there exists a class of $\mathrm{CV}$ s with a He-degenerate secondary that can have orbital periods down to a few minutes.
} 


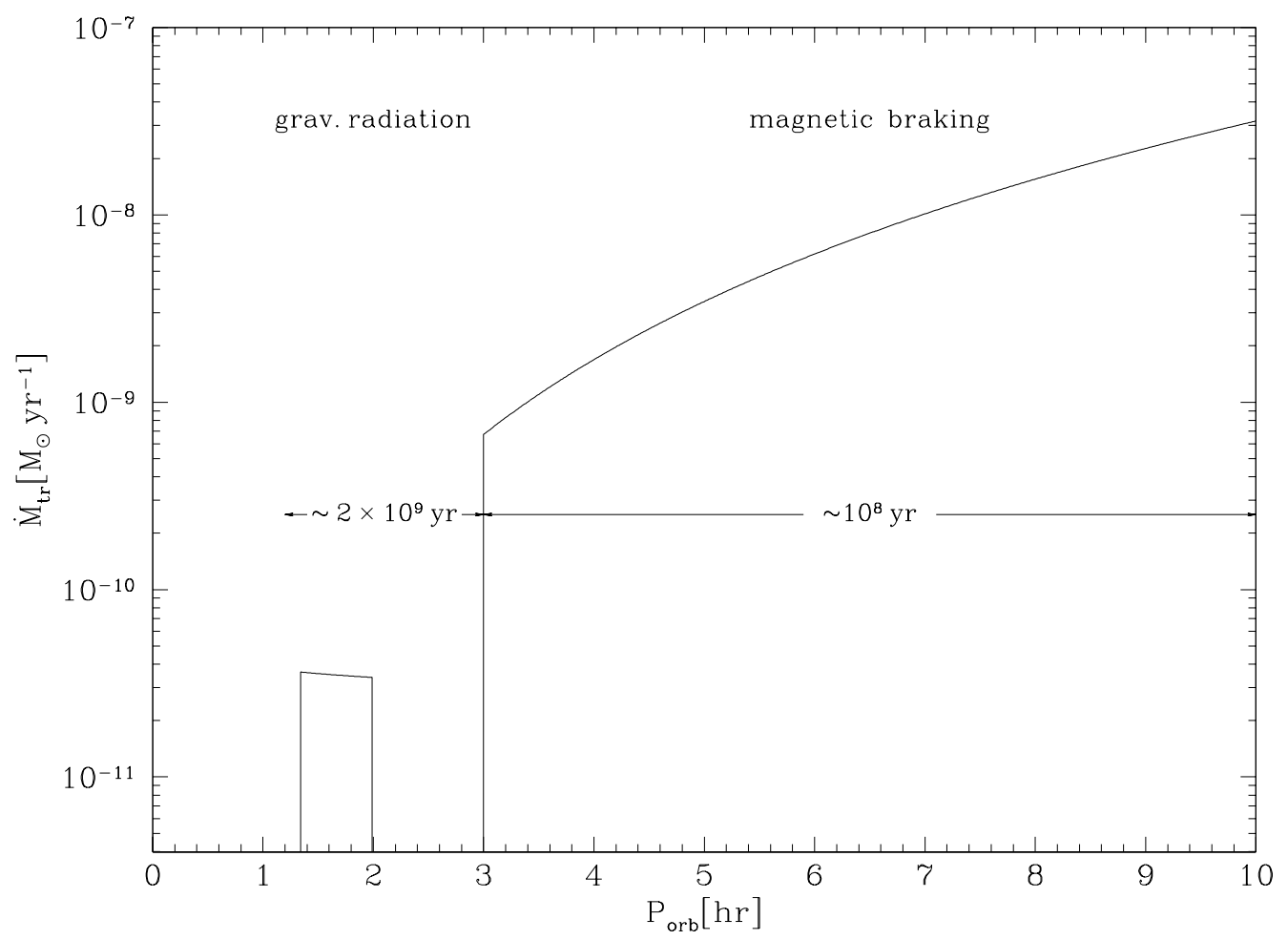

Figure 2.2: The standard CV evolution scenario in the mass transfer versus orbital period diagram. Angular momentum loss due to magnetic braking is believed to be the main mechanism above the period gap. As it is rather efficient, the binary evolves relatively fast to shorter orbital periods. Below $\sim 3 \mathrm{hr}$, gravitational radiation is the only process to lose angular momentum and it takes a relatively long time until the secondary becomes a brown dwarf.

and by Patterson (1984) for mass transfer caused by gravitational radiation below the period gap:

$$
\dot{M}_{\text {tr }}=3.81 \times 10^{-11} P_{\text {orb }}^{-1 / 6}(\mathrm{hr}) \frac{M_{\mathrm{wd}}^{2 / 3}}{(1-15 / 19 q)(1+q)^{1 / 3}} M_{\odot} \mathrm{yr}^{-1} .
$$

Here $q=\frac{M_{\mathrm{sec}}}{M_{\mathrm{wd}}}$ is the mass ratio of the stars and $P_{\mathrm{orb}}(\mathrm{hr})$ the orbital period in hours. For the white dwarf mass I used $M_{\mathrm{wd}}=1 M_{\odot}$ and neglected the variations of $q$ in Eq.(2.9) for simplicity. Detailed calculations can be found in Kolb \& Ritter (1992) and McDermott \& Taam (1989).

One of the main problems of the standard scenario, described above, results from the fact that there is a strong dispersion in mass transfer rates observed at a given 
orbital period (e.g. Warner 1995, 1987), whereas the theory predicts a definite value for the mass transfer rate for every orbital period (Fig. 2.2).

Many possible explanations for the dispersion in $\dot{M}_{\text {tr }}$ have been offered during the last decades, ranging from the influence of different evolutionary states of the secondary when it comes into contact with its Roche lobe (Pylyser \& Savonije $1988,1989)$ to magnetic activity which affects the flow in the vicinity of the $L_{1}$ point over a period of time (Barrett et al. 1989), or cyclical evolution with long periods of very low mass transfer, called hibernation (Shara 1989). In the context of this thesis the latter and the more recently suggested mass transfer cycles caused by irradiation of the secondary by parts of the accretion luminosity (King et al. 1995, 1996; McCormick \& Frank 1998; Ritter et al. 2000) will be discussed in more detail in chapter 9 . 


\section{Chapter 3}

\section{Theory of accretion discs}

It is rather complicated to describe the accretion of matter onto a compact object in full generality, because it requires solving multi-dimensional magnetohydrodynamic equations. The usual approach is to make assumptions about the nature of the accretion flows in order to simplify the equations.

The first solution for accretion onto a compact object was the so-called Bondisolution (Bondi 1952). This solution corresponds to the steady, radial, and spherically symmetric accretion of gas with zero angular momentum onto a central point mass. Most times the Bondi-solution is neither applicable for accretion from the interstellar medium nor in interacting binaries.

The thin disc model developed by Lynden-Bell \& Pringle (1974) and Shakura \& Sunyaev (1973) is the basis for most theoretical research of accretion discs.

\subsection{Thin discs}

The standard theory of geometrically thin accretion discs has been successfully applied to many properties of accreting systems. In this description several assumptions are made in order to derive the thin disc solution: the accretion flow is assumed to be axisymmetric, steady, geometrically thin, and without a vertical component of motion. In addition, it is common to parameterise the viscosity by assuming the radial-azimuthal component of the shear tensor being proportional to the total pressure (Shakura \& Sunyaev 1973). This is equivalent to the frequently used relation:

$$
\mathrm{v}=\alpha c_{s} H,
$$

where $v$ is the kinematic viscosity, and $H$ the pressure scale height and $0 \leq \alpha \leq 1$ the constant viscosity parameter. With these assumptions it is possible to obtain a 
self-consistent solution to the hydrodynamical equations of mass, angular momentum and energy conservation. In this solution the gas revolves in quasi-Keplerian orbits with a small radial drift velocity.

An important property of the thin disc is that the local dissipation rate is independent of the magnitude of viscosity. As the thin disc is assumed to be optically thick, each element radiates roughly as a blackbody with a temperature $T_{\text {eff }}$. By equating the emitted flux at every radius with the dissipation rate one obtains

$$
T_{\mathrm{eff}}^{4}=\frac{3 G M \dot{M}_{\mathrm{acc}}}{8 \pi \sigma R^{3}}\left(1-\sqrt{\frac{R_{\mathrm{in}}}{R}}\right),
$$

where $\sigma$ is the Stefan-Boltzmann constant, $G$ the gravitational constant, $R_{\text {in }}$ the radius of the inner edge of the disc, $R$ the radius of the disc annulus and $M$ the mass of the accreting star.

The thin disc solution has been the common paradigm in accretion theory for many years and is often called the standard accretion disc model (see Frank et al. 1992, for a detailed review).

\subsection{Shapiro-Lightman-Eardly solution}

In spite of the advantages of the thin disc solution it was noticed early that some objects containing a compact accreting component show hard X-ray emission even beyond $100 \mathrm{keV}$ which cannot be explained in the context of the thin disc solution. Following the suggestion of Thorne \& Price (1975), Shapiro et al. (1976) found a hot optically thin solution (SLE solution) by assuming that the energy generated viscously heats the protons which interact with the electrons only via Coulomb collisions. The resulting two temperature disc (protons $\sim 10^{11} \mathrm{~K}$; electrons $\sim$ $10^{9} \mathrm{~K}$ ) emits mainly Bremsstrahlung and naturally leads to hard X-ray emission. Unfortunately, the SLE solution is thermally unstable (Piran 1978) and therefore cannot be used to explain persistent hard X-ray emission.

\subsection{Advection dominated accretion flows}

Another problem of the standard accretion disc model is that in the case of very high accretion rates (which are, for example, required to reproduce the luminosities of Quasars) the solution becomes thermally unstable due to the strong tem- 
perature dependence of the pressure if

$$
\frac{P_{\text {gas }}}{P_{\text {tot }}}<\frac{5}{2}
$$

Here $P_{\text {gas }}$ is the gas pressure and $P_{\text {tot }}$ is the sum of gas and radiation pressure. The occurrence of this instability means that the standard accretion disc model is not able to describe the inner regions of bright AGN discs.

One of the most important processes that are not considered in the standard model of geometrically thin accretion discs and in the SLE-solution is advective cooling. As mentioned above it is assumed that the accreting gas cools so efficiently that all of the energy released through viscosity is radiated away locally. That this is not necessarily the case in the range of very high accretion rates in an optically thick accretion disc was first noticed by Katz (1977) and Begelman (1978). They showed that the diffusion time for photons can exceed the inflow time. Based on this seed, Abramowicz et al. $(1988,1996)$ constructed a global model for optically thick advection dominated accretion flows (ADAFs), often called "slim discs". These solutions are stable because significant parts of the generated energy are advected within the flow.

In addition to optically thick ADAFs or "slim discs" exist optically thin solutions of advection dominated accretion flows discovered by Ichimaru (1977). Many additional studies of optically thin ADAFs have been carried out since then (see e.g. Rees et al. 1982; Narayan \& Yi 1994, 1995). The strong promotion for optically thin ADAFs is motivated by the fact that these solutions can produce hard X-ray emission. In contrast to the SLE solution, optically thin ADAFs are thermally stable. As opposed to optically thick ADAFs, the optically thin solution only exists for relatively low accretion rates. According to the SLE solution it is assumed that the viscously generated energy affects the protons and that the electrons are heated solely via Coulomb collisions with the protons. The mechanism which leads to the dominance of advection in this case is the following: at low densities and high temperatures, Coulomb collisions become very inefficient and most of the energy is stored in the protons which cannot cool in an inflow time. The stored energy is thus advected within the flow. Interestingly, Narayan \& Yi (1994) found that parts of the flow are able to escape to infinity because of the stored energy. This result was taken into account by Blandford \& Begelman (1999) who derived self-similar advection dominated inflow-outflow solutions (ADIOS). In spite of these findings, it is rather fair to say that the connection between optically thin advection dominated accretion flows and mass loss via winds remains an open question.

Moreover, Bisnovatyi-Kogan \& Lovelace (2000) found that the assumptions underlying the optically thin ADAF solutions ignore the effects of magnetic field 


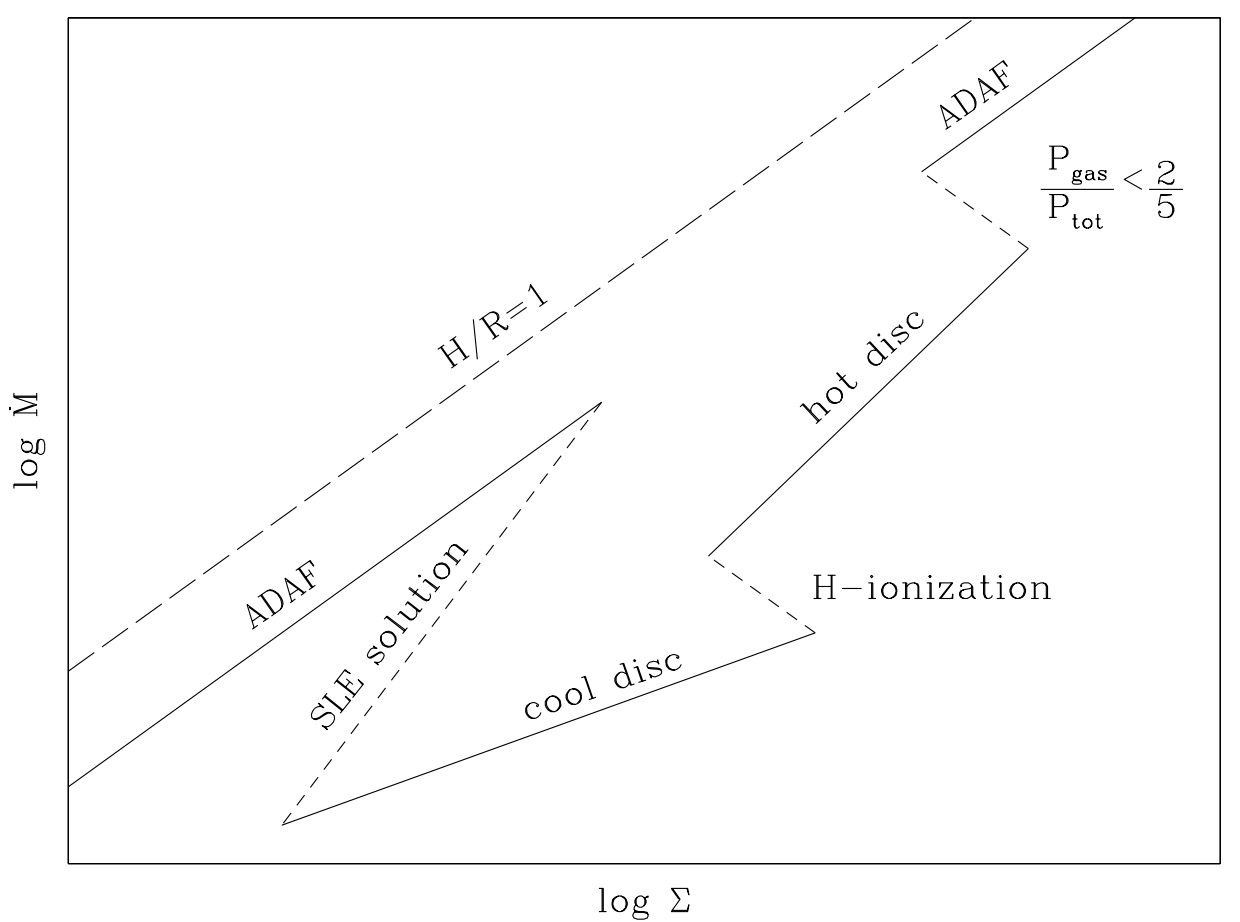

Figure 3.1: A schematic view of the thermal equilibrium curves of accretion flow solutions in the accretion rate $\dot{M}$-surface density $\Sigma$ diagram. From left to right: optically thin ADAF solutions exist until the surface density reaches a value where energy transfer via Coulomb is sufficiently efficient and the energy is no longer advected but radiated away. The second optically thin solution is the SLE solution which is thermally unstable. For optically thick standard discs there exist two branches (cool and hot) depending on the ionisation state of the hydrogen in the disc. These two stable branches are separated by a thermally unstable branch in the region of partial ionisation of hydrogen. The hot standard solution again becomes thermally unstable for high accretion rates and surface densities when radiation pressure becomes dominant. A stable advection dominated solution, often called the "slim disc" solution, exists for very high accretion rates.

reconnection which might heat up the plasma of the flow. This contribution is completely neglected by taking into account only electron heating due to Coulomb collisions with ions. Bisnovatyi-Kogan \& Lovelace (2000) analysed the physical processes in optically thin accretion flows at low accretion rates, including the influence of an equipartition random magnetic field and the heating of electrons due to magnetic field reconnection. They found that such heating significantly restricts the applicability of ADAF solutions, and that it leads to a radiative efficiency of the flows of $\sim 25 \%$ of the standard accretion disc value. 


\subsection{Thermal equilibrium and stability}

Fig. 3.1 shows schematically the thermal equilibrium curves (cooling equates heating) for the solutions of accretion flows mentioned above. The accretion rate $\dot{M}$ is plotted against the surface density $\Sigma$, i.e. the vertically integrated density at a given radius.

The course of the equilibrium curve can be quantitatively understood by considering the relevant heating $\left(Q_{+}\right)$and cooling (radiative $Q_{-}$and advective $Q_{\mathrm{adv}}$ ) terms. Of particular importance are the S-shapes on the right hand side of Fig. 3.1 where three equilibrium solutions for a given surface density can be found. For the lower S-shape, which occurs in the region of partial ionisation of hydrogen and which connects the cold and the hot standard solution, gas pressure is the dominant pressure term. On the cold branch both the cooling and the heating rates increase with increasing accretion rate. The temperature of the disc increases as well as the surface density. For temperatures $\sim 6 \times 10^{3} \mathrm{~K}$ the ionisation of hydrogen starts, the opacity increases dramatically with the temperature, leading to reduced cooling of the disc (the viscously generated energy goes into the ionisation of hydrogen). The only possibility to maintain thermal equilibrium is to decrease the surface density which leads to reduced heating and to increased cooling. If the disc is fully ionised, the strong temperature dependence of the opacity vanishes and heating equals cooling while the surface density increases with increasing accretion (hot standard solution). The schematic view presented in Fig. 3.1 is of course not the whole story and I will explain the course of the thermal equilibrium curve in the region of partial ionisation of hydrogen in detail in the next section.

The upper S-curve in Fig. 3.1 describes the case when radiation pressure becomes dominant and is not of importance for this thesis because the accretion rates in $\mathrm{CV}$ discs are rather low. The heating rate is proportional to the pressure in the $\alpha$ prescription. Therefore, the temperature dependence of the heating rate switches from $\propto T$ to $\propto T^{4}$ when radiation pressure becomes important. The resulting drastic increase of the heating rate is compensated by a decrease of the surface density leading to increased cooling. When the accretion rate reaches a value for which advection significantly contributes to the cooling term $\left(Q_{+}=Q_{-}+Q_{\text {adv }}\right)$, the surface density starts to increase with increasing accretion (for a more detailed discussion see e.g. Kato et al. 1998, chapter 10).

In Fig. 3.1 thermally unstable regions are plotted with short dashed lines. The Sshapes and the instability of the middle branches offer the possibility to construct a limit-cycle behaviour in which the accretion discs are switching between the upper and the lower stable solution in order to match a given accretion rate inbetween. To describe such a limit-cycle behaviour it is necessary to develop a 
time-dependent model for the accretion disc.

The dwarf nova outbursts observed in many CVs are believed to result from such a limit-cycle behaviour of the accretion disc. In the next chapter I describe this theory, discuss its applications to dwarf nova outbursts and present simulations of dwarf nova outbursts using a Finite-Element code. 


\section{Chapter 4}

\section{Dwarf nova outbursts and disc instabilities}

The viscous thin disc solution is now roughly thirty years old and well known. In spite of this relatively long time, due to the advantages and the success of this description it has remained in the focus of research up to now. Especially the timedependent vertically averaged description has linked observations and theory very successfully. As its goal is to explain the dwarf nova outbursts observed in many CVs it plays a central role in this thesis.

In the following sections I describe my model of time-dependent disc accretion onto white dwarfs. The results of the vertical structure shown in Figs. 4.2-4.6 are obtained using the vertical structure code written by J.K. Cannizzo who kindly provided me with a copy of his code.

\subsection{Assumptions and time scales}

In section 3.1 I reviewed briefly the properties of the steady thin disc solution. Here I derive the equations describing the time-dependence of thin accretion discs using the following assumptions:

$$
\begin{aligned}
\frac{H}{R} & \ll 1, \\
v_{z} & =0, \\
\frac{\partial}{\partial \phi} & =0, \\
\frac{\partial v_{R}}{\partial z}=\frac{\partial v_{\phi}}{\partial z} & =0,
\end{aligned}
$$


where $H$ is the pressure scale height, $R$ the radius, and $v_{z}, v_{\phi}$ and $v_{R}$ are the components of the velocity. Thus, the disc is assumed to be geometrically thin and azimuthally symmetric, the vertical velocity is set equal to zero and the other components of the velocity are assumed to depend only on the radius.

In addition, it is common to assume that the gas in the disc moves with quasiKeplerian angular velocities. This is equivalent to the assumption that the radial pressure gradient is negligible and that the radial velocity is small compared to the angular velocity. It is possible to show that this is true in a steady thin disc because the thin disc condition leads to: (1) supersonic circular velocities, and small subsonic radial drift velocities, i.e.

$$
v_{R} \ll c_{s} \ll v_{\phi},
$$

and (2) to the insignificance of the radial pressure term compared to the gravity term in the radial component of the Navier-Stokes equation (see Frank et al. 1992, for a detailed discussion).

With the assumptions above one obtains from the basic hydrodynamic equations describing the conservation of mass, angular momentum and energy the following set of equations:

$$
\begin{aligned}
& \frac{\partial \rho}{\partial t}+\frac{\partial\left(\rho v_{\mathrm{R}}\right)}{\partial R}+\frac{\rho v_{\mathrm{R}}}{R}=0, \\
& \rho \frac{v_{\phi}^{2}}{R}=-\rho \frac{G M_{*}}{R^{2}}, \\
& \frac{\partial P}{\partial z}=\rho \Omega_{K}^{2} z \\
& \rho\left(\frac{\partial v_{\phi}}{\partial t}+v_{\mathrm{R}} \frac{\partial v_{\phi}}{\partial R}+\frac{v_{\mathrm{R}} v_{\phi}}{R}\right)=\eta\left(\frac{\partial^{2} v_{\phi}}{\partial R^{2}}+\frac{1}{R} \frac{\partial v_{\phi}}{\partial R}-\frac{v_{\phi}}{R^{2}}\right) \\
& +\frac{\partial \eta}{\partial R}\left(\frac{\partial v_{\phi}}{\partial R}-\frac{v_{\phi}}{R}\right) \\
& c_{\mathrm{v}}\left(\rho \frac{d T}{d t}-\left(\Gamma_{3}-1\right) T \frac{d \rho}{d t}\right)=\eta\left(R \frac{\partial \Omega_{\mathrm{K}}}{\partial R}\right)^{2}+\frac{\partial F_{\mathrm{Z}}}{\partial z}+\frac{1}{R} \frac{\partial\left(R F_{\mathrm{R}}\right)}{\partial R}
\end{aligned}
$$

where $\rho$ is the density, $P$ the pressure, $c_{\mathrm{V}}$ the specific heat, $\Omega_{\mathrm{K}}$ the Keplerian angular velocity, $\Gamma_{3}$ is the ratio of specific heats, and $\eta$ the dynamic viscosity. $F_{\mathrm{Z}}$ and $F_{\mathrm{R}}$ are the vertical and radial radiative flux, respectively.

Eq. (4.6) results from conservation of mass whereas Eqs. (4.7)-(4.9) describe the radial, vertical and tangential component of angular momentum conservation. Eq. (4.10) follows from energy conservation. 
In a steady $\left(\frac{\partial}{\partial t}=0\right)$ thin disc, both temperature and pressure gradient are essentially vertical and $F_{R} \ll F_{z}$. Therefore, the vertical and radial structure are largely decoupled.

In order to describe the time-dependent behaviour of the accretion disc it is useful to take a look at the relevant timescales on which the structure of the disc is changing:

$$
\begin{array}{r}
t_{\mathrm{visc}} \sim \frac{R}{v_{\mathrm{R}}}, \\
t_{\phi} \sim \Omega_{\mathrm{K}}^{-1}, \\
t_{\mathrm{z}} \sim \frac{R}{M c_{\mathrm{s}}} \sim t_{\phi}, \\
t_{\mathrm{th}} \sim \frac{c_{\mathrm{s}}^{2}}{v_{\phi}^{2}} t_{\mathrm{visc}},
\end{array}
$$

where $M$ is the Mach-number. In the thin disc approximation the viscous time scale $t_{\text {visc }}$ is significantly longer (of the order of several days) than the other time scales (of the order of minutes). Therefore, the changes of the density in the radial direction are slow enough to maintain hydrostatic equilibrium in the vertical direction at every moment. So it is possible to use stationary equations for the vertical structure, connected with time-dependent equations refering to the conservation of angular momentum, energy and mass in the radial direction, in order to describe the viscous evolution of a thin disc. In addition one has to make an assumption about the viscosity. The standard scaling of the viscosity is called the $\alpha$-prescription:

$$
t_{r \phi}=\eta \frac{3}{2} \Omega_{\mathrm{K}}=\alpha P
$$

where $P$ is the sum of gas and radiation pressure, I used the assumption $\Omega=\Omega_{\mathrm{K}}$ and $t_{r \phi}$ is the radial-azimuthal component of the shear tensor (Shakura \& Sunyaev 1973). It is interesting to note that although the source of the viscosity, which is parameterised with $\alpha$ in Eq. (4.15), is rather uncertain, it is quite clear that molecular viscosity is far too weak to bring about the dissipation and angular momentum transport required to explain the observed phenomena. Considerable effort has been expended on seeking the physical mechanism behind $\alpha$. The developed theories can be devided into three subclasses: turbulence, magnetic stresses, and collective hydrodynamik effects (see Frank et al. 1992; Warner 1995, and references therein). In spite of these studies, consistency between phenomenology and theory is not yet obtained. Therefore, we need both, further refinement of the theories and more detailed $\alpha$-models in comparison with observations to discover the nature of $\alpha$. This thesis contributes to the latter. 


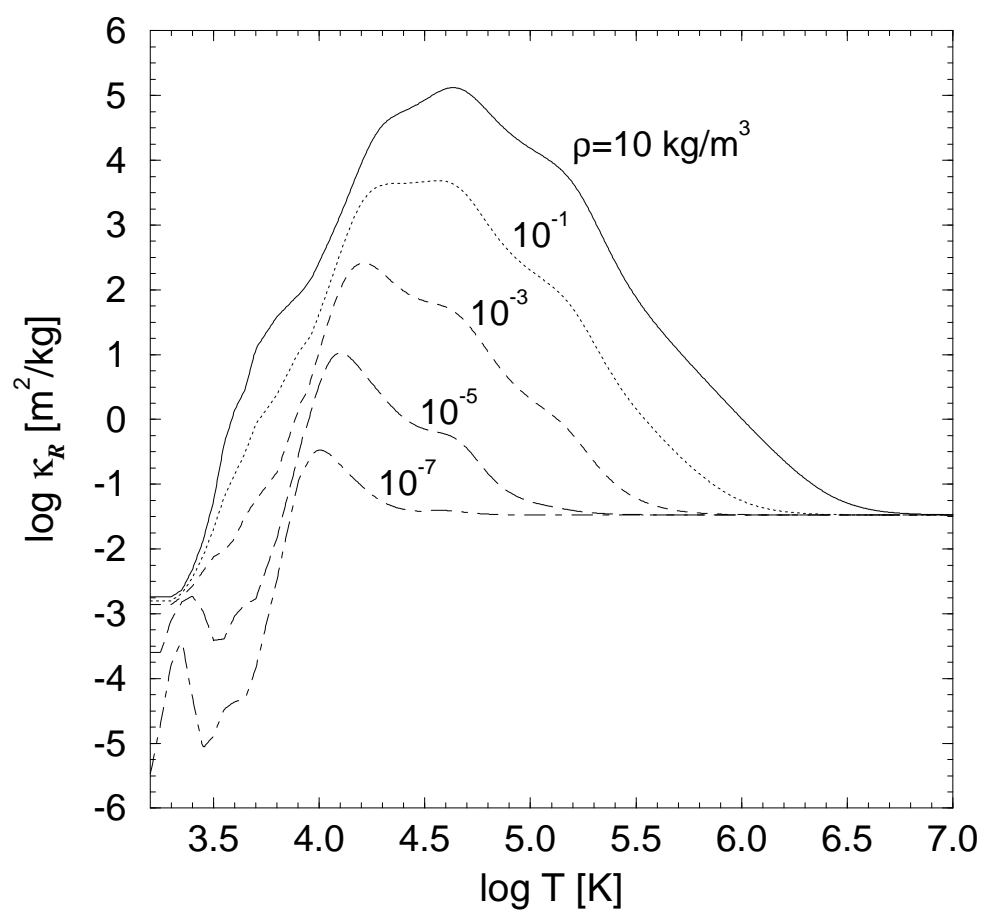

Figure 4.1: The Rosseland mean opacity $\kappa_{R}$ for solar abundances as a function of temperature and for different densities.

\subsection{Vertical structure}

\subsubsection{The equations}

The equations describing the vertical structure of accretion discs in the standard picture are very similar to the vertical equations describing the stellar interior structure (e.g. Kippenhahn 1967). I reiterate the basic equations for the vertical structure of a thin, Keplerian disc obtained from hydrostatic equilibrium, energy transport, flux generation and mass conservation (e.g. Lynden-Bell 1969; LyndenBell \& Pringle 1974; Shakura \& Sunyaev 1973; Pringle 1981). These equations result from Eqs (4.8) and (4.10) by assuming steady accretion, considering only the vertical components, using the $\alpha$-prescription and standard equations for energy transport. They may be written as:

$$
\begin{gathered}
\frac{d P}{d z}=-\rho \Omega_{\mathrm{K}} z \\
F_{z}=F_{\mathrm{rad}}+F_{\text {conv }}, \quad \nabla_{*}>\nabla_{\mathrm{ad}},
\end{gathered}
$$




$$
\begin{gathered}
F_{\text {conv }}=c_{p} \rho \sqrt{\frac{g_{z}}{T}} \frac{l^{2}}{4}\left(\left|\frac{\partial T}{\partial z}\right|-\left|\frac{\partial T}{\partial z}\right|_{\mathrm{ad}}\right)^{\frac{3}{2}}, \\
F_{z}=F_{\mathrm{rad}}=-\frac{4}{3} \frac{a c}{\kappa_{\mathrm{R}} \rho} T^{3} \frac{d T}{d z}, \quad \nabla_{*}<\nabla_{\mathrm{ad}}, \\
\frac{d F}{d z}=\eta\left(R \frac{\partial \Omega_{\mathrm{K}}}{\partial R}\right)^{2}=\frac{3}{2} \alpha \Omega_{\mathrm{K}} P,
\end{gathered}
$$

where $\nabla_{*}=\left(\frac{d \ln T}{d \ln P}\right)_{\mathrm{rad}}, \nabla_{\mathrm{ad}}=\left(\frac{d \ln T}{d \ln P}\right)_{\mathrm{ad}}, l$ denotes the mixing length, $g_{z}$ contains both, the contributions of the primary and the self-gravity of the disc and $a=$ $\frac{4 \sigma}{c}$. In Eq. (4.19) $\kappa_{\mathrm{R}}$ denotes the Rosseland mean opacity which has a strong temperature dependence in the region of partially ionised hydrogen as displayed in Fig 4.1. It is common and useful to define the surface density coordinate $\Sigma_{z}$ by

$$
\frac{d \Sigma_{\mathrm{z}}}{d z}=2 \rho,
$$

or equivalently $\Sigma_{z}(z)=\int_{-z}^{z} \rho d z$. The system is comlete with the equation of state, i.e.

$$
P=P_{\text {gas }}+P_{\text {rad }}=\frac{\rho R_{g} T}{\mu}+\frac{1}{3} a T^{4}
$$

and an assumption about the mixing length. Throughout this thesis I use

$$
l=\min (z, H) .
$$

The more common relation for the mixing length, $l=\alpha_{\text {mix }} H$, with $1 \leq \alpha_{\text {mix }} \leq 2$, is replaced by Eq. (4.23) in order to get a finite value for $l$ even at the disc midplane.

As the inner boundary conditions are known only for the surface density, the height and the flux, whereas the outer boundary conditions are only given for the pressure, temperature and flux, the system of Eqs. (4.16-4.23) defines a socalled two point boundary value problem which can be solved with the relaxation method (Cannizzo \& Cameron 1988). To do so, the variable $z$ is replaced by the flux $F_{z}$ and the vertical structure is distributed over a grid of $N$ points where the innermost point $i=1$ is placed at the midplane and the last point $i=N$ is placed at the photosphere.

The input parameters for the problem are the central object mass $M_{\mathrm{wd}}$, the inner disc radius $R_{\text {in }}$, the radial distance to the point of interest $R$ and the accretion rate $\dot{M}_{\text {acc }}$. These are related to the surface flux by

$$
T_{\mathrm{eff}}^{4}=\frac{3 G M_{\mathrm{wd}} \dot{M}_{\mathrm{acc}}}{8 \pi \sigma R^{3}}\left(1-\sqrt{\frac{R_{\mathrm{in}}}{R}}\right)
$$

(Shakura \& Sunyaev 1973). 


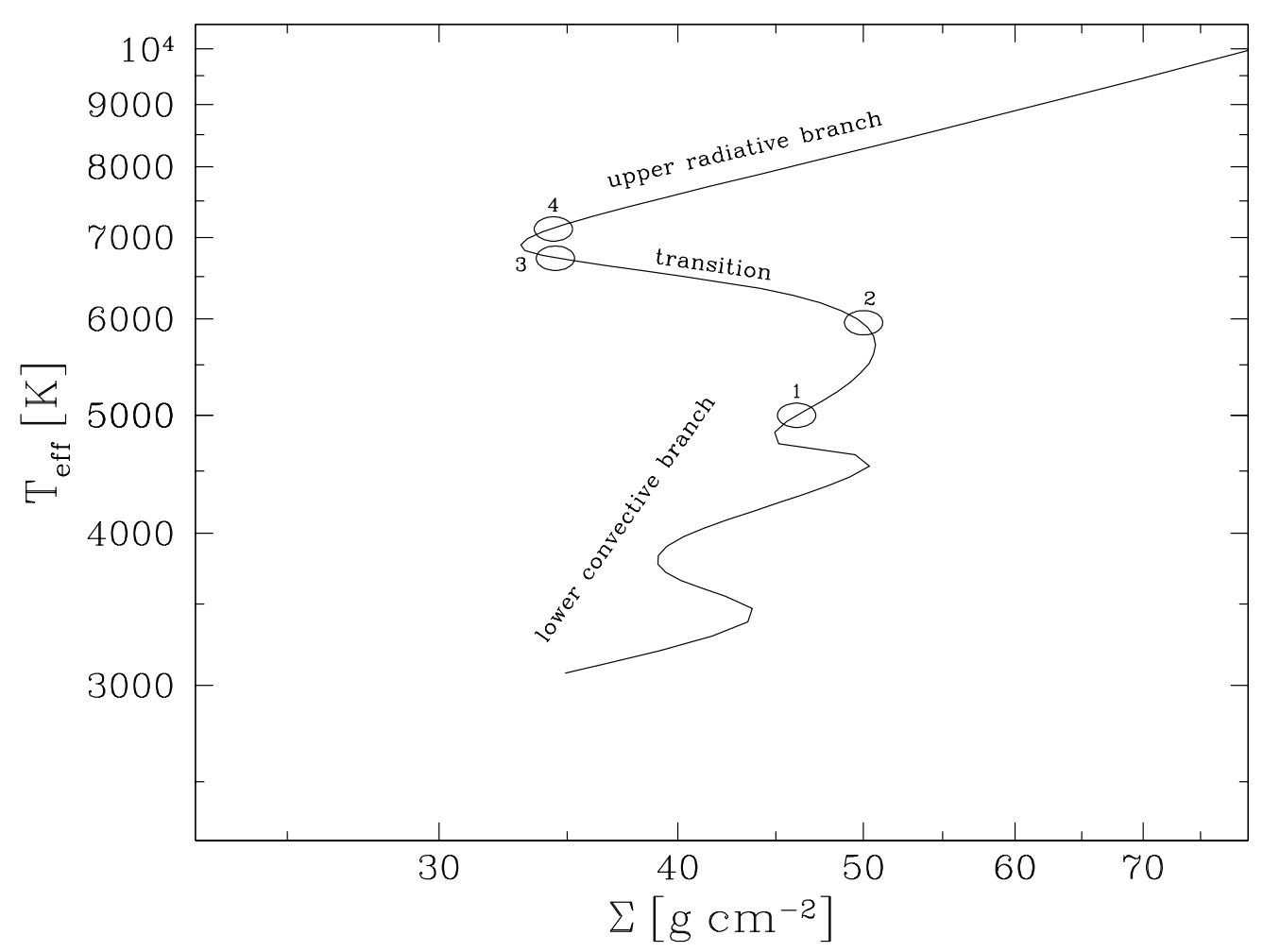

Figure 4.2: A sequence of solutions for the vertical structure in the $\Sigma-T_{\text {eff }}$ plane at a radius of $R=1.3 \times 10^{10} \mathrm{~cm}$, for a white dwarf mass of $1.1 M_{\odot}$ and $\alpha=0.2$. The circles mark the solutions for which the vertical structure is presented in Fig. 4.3. The regions which are called lower, middle and upper branch in the text are indicated as lower convective branch, transition, and upper radiative branch.

\subsubsection{The S-curve in the $\Sigma$ - $T_{\text {eff }}$ diagram}

As done in the schematic picture Fig. 3.1, it is common to present a series of steady state solutions of the vertical structure in the $\dot{M}_{\text {acc }}$ or $T_{\text {eff }}$ versus $\Sigma$ diagram. By changing $\dot{M}_{\text {acc }}$ one successively obtains a family of solutions for fixed values of $\alpha, R$, and $M_{\mathrm{wd}}$. Fig. 4.2 shows an example of such an equilibrium curve. The found hysteresis relation - the so-called S-curve - displays the base of the thermal instability model. There exist at least three solutions for a certain range of $\Sigma$. Hydrogen is fully ionised in the high temperature branch (hot disc in Fig. 3.1 and the upper radiative branch in Fig. 3.2), partially ionised in the middle branch and nearly neutral in the low temperature branch (cold disc in Fig. 3.1 and the lower convective branch in Fig. 3.2). Hence, the ultimate cause of the S-shape is the 


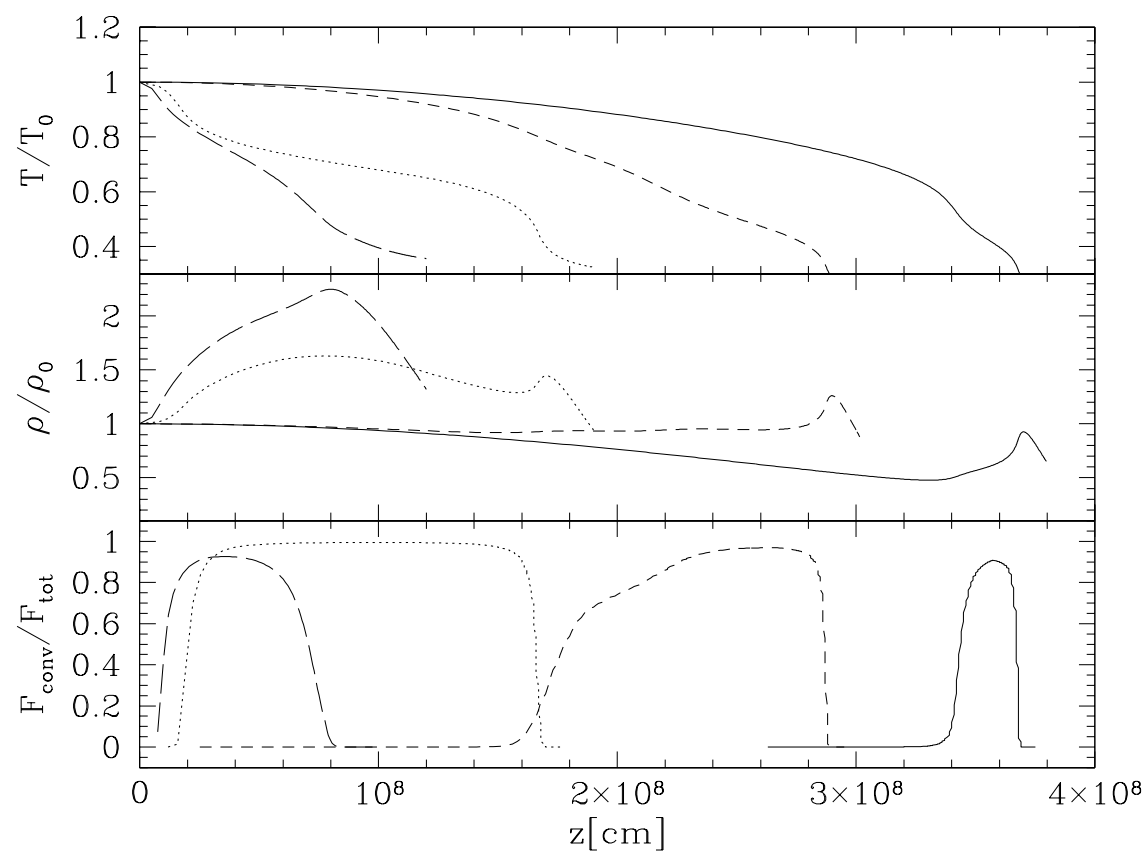

Figure 4.3: Normalised vertical structure results for the temperature, the density, and the ratio of convective to total energy transport. The solid line corresponds to the circle (4) of the upper branch in Fig. 4.3, the short dashed and the dotted line represent the solution at the beginning (3) and the end (2) of the unstable middle branch respectively and the long dashed lines display the solution in the cold state (1). Above the unstable middle branch convection acts only near the photosphere. At the middle branch convection becomes more and more important until the disc is fully convective and becomes stable.

partial ionisation of hydrogen at $\sim 10^{4} \mathrm{~K}$.

The equilibrium curve presented in Fig. 4.2 is obviously more complex than a simple $S$. The lower convective branch of the equilibrium curve shows a somewhat wavy line containing two additional S-shapes. In the following I distinguish between the upper $S$-curve which refers to the general S-shape of the upper, middle and lower branch, and the lower $S$-curve which refers to the wavy character of the lower convective branch (see Fig. 4.2).

There are different physical effects which produce the upper and the lower Scurve. The wavy shape of the lower branch of the equilibrium curve is produced by the strong temperature dependence of the opacity around $2200 \mathrm{~K}$ and in the region of partially ionised hydrogen at $10^{4} \mathrm{~K}$ (see Fig. 4.1). The occurrence of the lower S-curve can be understood by taking into account the consequences of the thin disc approximation and the $\alpha$-prescription. As $T_{c}$ greatly exceeds the surface 


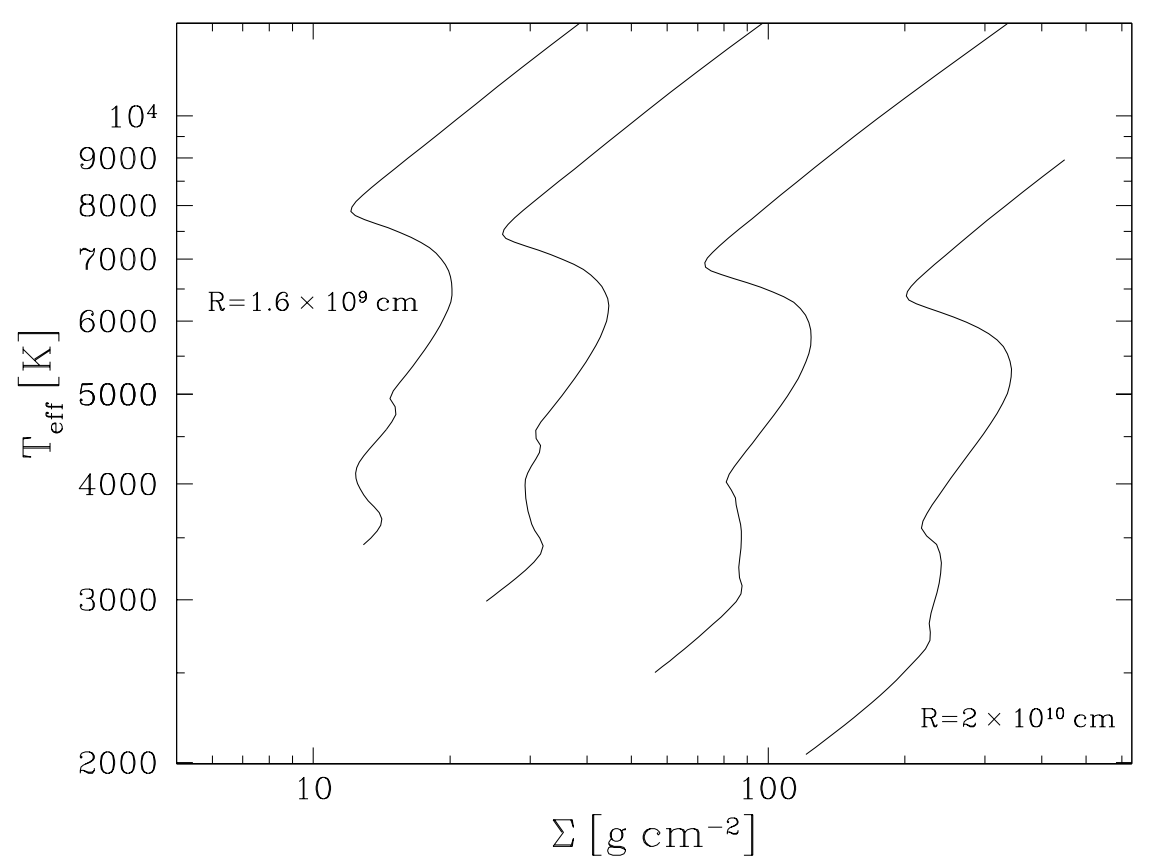

Figure 4.4: The dependence of the S-curve on the disc radius. I used $M_{\mathrm{wd}}=0.5 M_{\odot}$ and $\alpha=0.05$. The maximum effective temperature of the lower branch decreases with increasing radius.

temperature $T_{\text {eff }}$ one obtains:

$$
\dot{M}_{\mathrm{acc}} \propto T_{\mathrm{eff}}^{4} \propto v \Sigma \propto \alpha T_{\mathrm{c}} \Sigma
$$

Therefore, in case $T_{\mathrm{c}}$ increases faster than $T_{\text {eff }}$ and $\alpha$ is constant, $\Sigma$ must decrease with increasing $T_{\text {eff }}$. For $T \sim 7000 \mathrm{~K}$ we have $\kappa_{\mathrm{R}} \sim T^{10}$. Neglecting convection, one obtains $\kappa_{\mathrm{R}} \Sigma T_{\mathrm{eff}}^{4} \sim T_{\mathrm{c}}^{4}$ and, thus, $\Sigma T_{\mathrm{eff}} \sim T_{\mathrm{c}}^{-6}$.

The upper S-curve is brought about by convection. The same partial ionisation which gives rise to the large opacity also leads to a large heat capacity as the addition or subtraction of energy goes almost exclusively into changing the ionisation level of the gas and produces only a small change in temperature. Following the upper branch downward to smaller effective temperatures, the specific heats, $c_{\mathrm{p}}$ and $c_{\mathrm{V}}$ both increase while their difference remains constant, the ratio $\gamma=\frac{c_{\mathrm{p}}}{c_{\mathrm{v}}} \mathrm{de}$ creases and $\nabla_{\text {ad }}$ becomes small. As a result, for $T_{\text {eff }} \lesssim 8000 \mathrm{~K}$ convection gets increasingly strong and the central (or midplane) temperature is reduced. This leads to a steep dependence of $T_{\mathrm{c}}$ on $T_{\text {eff }}$ and to a range where $\frac{d \Sigma}{d T_{\text {eff }}}<0$, i.e. the middle transition branch of the upper S-curve. When convection carries most of the energy there can no longer be sensitivity to the fraction of convective heat 


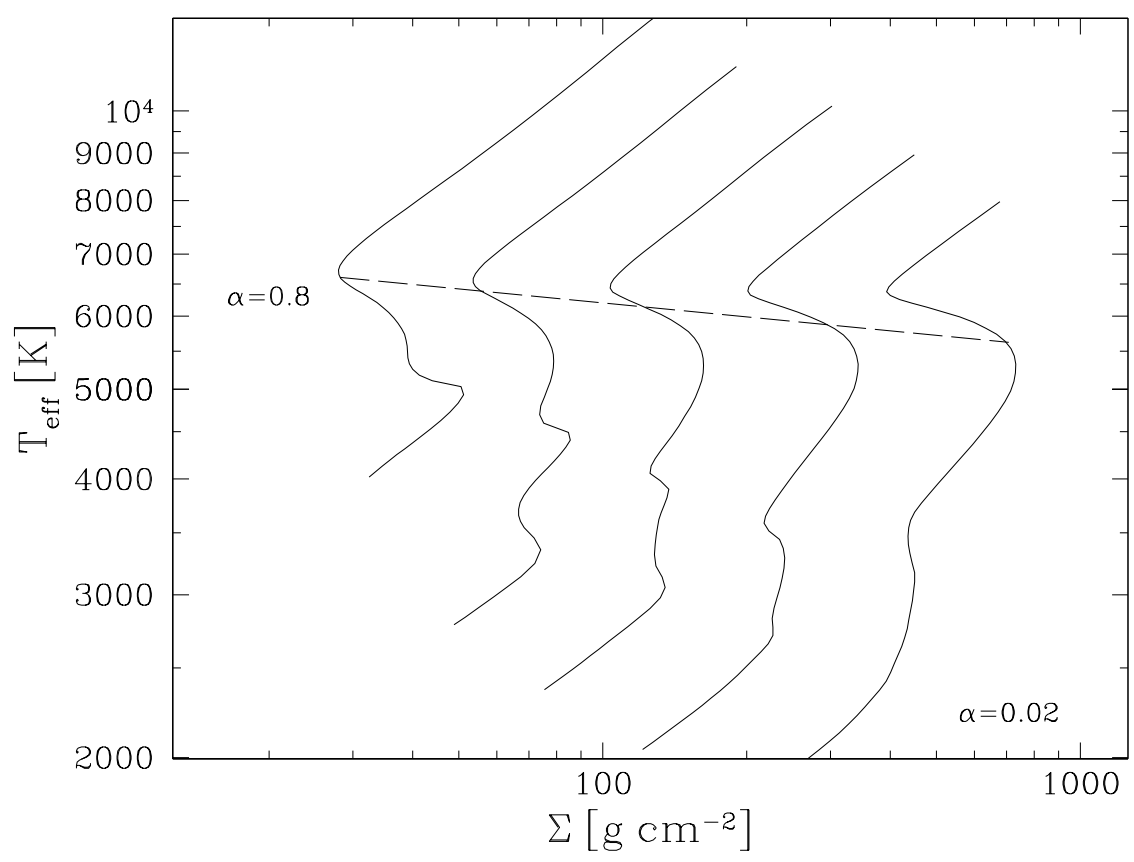

Figure 4.5: The dependence of the S-curve on the viscosity parameter $\alpha$. The white dwarf mass and the radius are: $M_{\mathrm{wd}}=0.5 M_{\odot}, R=2 \times 10^{10} \mathrm{~cm}$. The dashed line connects the upper and the lower branch for different values of $\alpha$ because it is necessary to use different values at the upper and the lower branch, $\alpha_{\mathrm{h}}$ (upper branch) and $\alpha_{\mathrm{c}}$ (lower branch), in order to reproduce observed dwarf nova outbursts (see section 4.4). Typical values of $\alpha_{h}$ and $\alpha_{\mathrm{c}}$ are: $\alpha_{\mathrm{h}} \sim 10^{-1}, \alpha_{\mathrm{c}}=2 \times 10^{-2}$

transport and, hence, to the shape of the equilibrium curve in the $\Sigma-T_{\text {eff }}$ plane. Thus the accretion rate (or effective temperature) at which the disc is fully convective defines the lower maximum of the upper S-curve.

Figs. 4.2 and 4.3 show a sequence of solutions in the $\Sigma-T_{\text {eff }}$ plane and the vertical structure for the positions marked with circles.

Figs. 4.4 and 4.5 display the equilibrium relation among the effective temperature and the surface density for different values of radii and $\alpha$ respectively. Each curve has an S-shape. The wavy character of the lower branch reduces somewhat with decreasing $\alpha$ and radius, whereas the effective temperature at which the upper S-curve occurs is nearly independent of $\alpha$ and is only slightly decreasing with radius. 


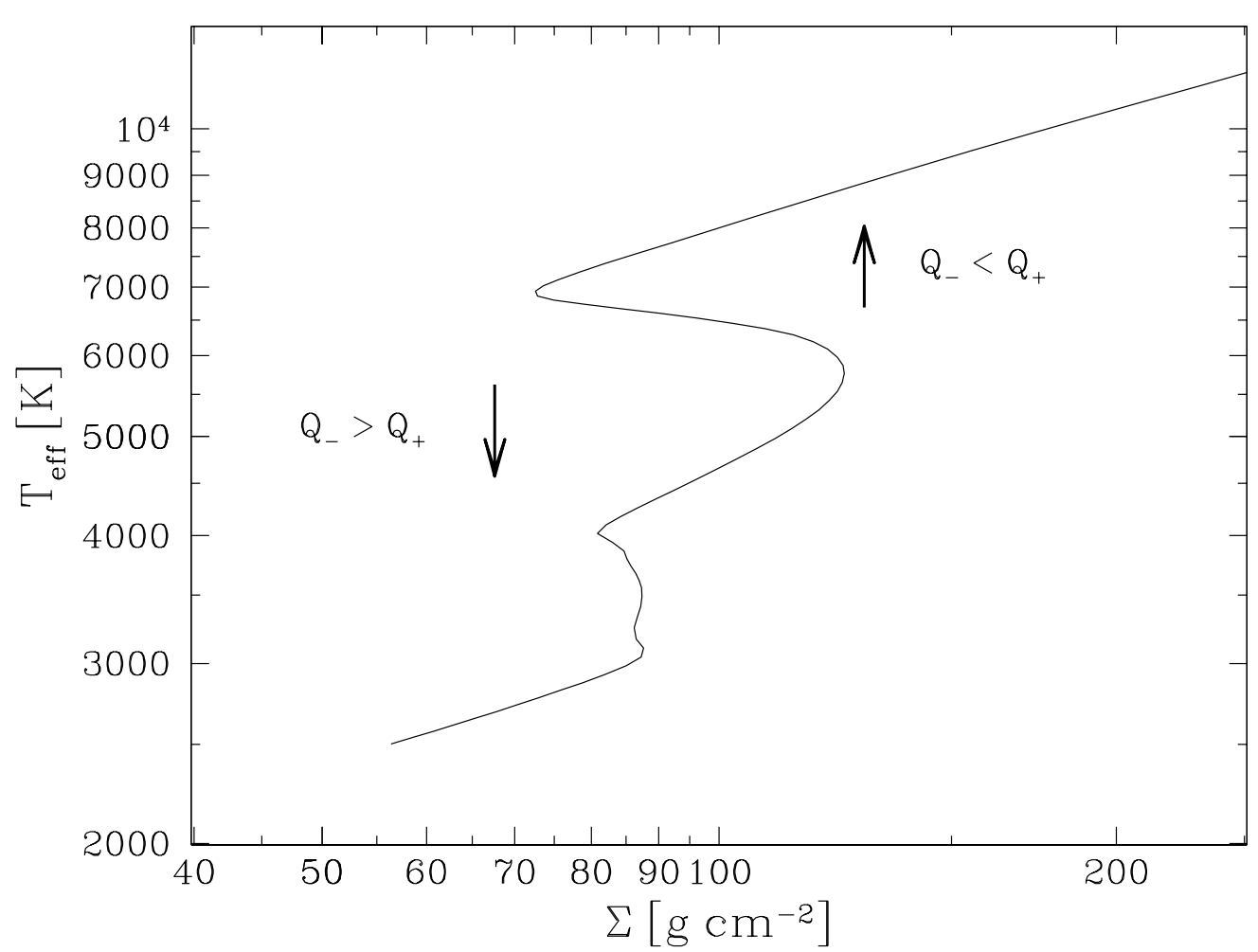

Figure 4.6: The limit-cycle behaviour of an annulus of the accretion disc. If $\Sigma$ exceeds its critical value on the lower branch, the disc relaxes to thermal equilibrium on the hot ionised branch. As the mass transfer from the neighbouring ring is less than the accretion rate through the annulus, the surface density decreases until the critical value of the upper branch is reached and the annulus relaxes to the cold equilibrium value.

\subsubsection{Instabilities and the thermal limit-cycle}

The famous S-curves resulting from a series of calculations of the vertical structure for a given radius, $\alpha$, and $M_{\mathrm{wd}}$ represent the thermal equilibrium, i.e. the locus where viscous heating is balanced by radiation from the surface $\left(Q_{-}=Q_{+}\right)$. For values ( $\left.\Sigma, T_{\text {eff }}\right)$ which place an annulus on the right (left) of the equilibrium curve heating (cooling) exceeds cooling (heating). Consequently the annulus will heat up (cool down) until it reaches the equilibrium curve.

The key-ingredient of the limit-cycle model lies in the thermal instability of the middle branch of the S-curve, where $\frac{d T_{\text {eff }}}{d \Sigma}<0$. This is easy to understand by considering the description of the thermal equilibrium curve above. Once an annulus is placed at the middle branch and is pushed somewhat out of equilibrium, say 
to a lower surface density, this results in decreased heating but leads to increased cooling as the new equilibrium would be at a higher effective temperature. i.e. As a consequence any small discrepancy between cooling and heating grows: the annulus is thermally unstable.

The middle branch is also viscously unstable because the S-curve is also present in the $\dot{M}_{\text {acc }}-\Sigma$ plane (see Eq. (4.24)). Any increase of the surface density leads to a decrease of $\dot{M}_{\text {acc }}$, which further increases the surface density of the annulus (Lightman \& Eardley 1974). Note, that this instability operates on the viscous timescale which is much longer than the thermal timescale (see Eqs. (4.11) and (4.14)).

Obviously, the S-curve defines two critical values for the accretion rate (equivalently for the effective temperature) for every disc annulus between which no stable disc solution exists. If we consider the case that the rate at which matter is transferred from the secondary to the accretion disc via the accretion stream $\left(\dot{M}_{\mathrm{tr}}\right)$ lies between these two critical values for the outer edge of the disc, there is no equilibrium available for the outer annulus. Assuming the annulus to lie on the upper branch, the condition $\dot{M}_{\text {acc }}>\dot{M}_{\text {tr }}$ leads to a decrease of $\Sigma$ until it reaches the minimum value for the lower branch of the $S$-curve. At this point the annulus cannot follow the S-curve into the middle branch but instead cools down on the thermal timescale because cooling exceeds heating and finds its equilibrium on the lower cold branch of the S-curve. Now the annulus transfers less mass than arrives from the secondary which increases its surface density $\Sigma$ but decreases the surface density of the neighbouring annulus. This leads to a cooling front which brings the entire disc into the cold state with a very low mass transport through the disc. As a consequence, the mass arriving from the secondary accumulates in the disc until somewhere in the disc $\Sigma$ reaches the critical value of the lower branch so that the corresponding annulus heats up on the thermal timescale $\left(Q_{-}<Q_{+}\right)$. The increase of viscosity at this annulus leads to an increased mass transfer resulting in increased surface densities for the neighbouring annuli. A heating front turns the entire disc back into the hot ionised state.

In order to match the given value of the mass transfer rate from the secondary $\dot{M}_{\text {tr }}$ the disc switches between two states. In the hot state the accretion rate onto the white dwarf exceeds the mass transfer rate from the secondary $\dot{M}_{\text {acc }}>\dot{M}_{\text {tr }}$, whereas the disc transports less mass in the cold state $\dot{M}_{\text {acc }}<\dot{M}_{\text {tr }}$. This limitcycle behaviour constitutes the key aspect of the thermal limit-cycle model and the theory of dwarf nova outbursts. Fig. 4.6 displays the limit-cycle behaviour for a disc annulus at $R=2 \times 10^{10} \mathrm{~cm}$ and $\alpha=0.05$. 


\subsection{The vertically averaged description}

After decoupling the vertical and the radial structure I presented solutions of the vertical structure (section 4.2). These solutions have to be connected with the radial evolution to obtain a model for time-dependent accretion discs. In order to achieve this goal it is common to transform the equations describing the evolution of the disc into one-dimensional equations by averaging over the vertical structure. The surface density equals the density integrated over $z$ :

$$
\Sigma=\int_{-\infty}^{\infty} \rho d z
$$

Following the standard thin disc approximation I write $\Sigma=2 \rho_{\mathrm{c}} H$ with $H$ and $\rho_{\mathrm{c}}$ being the pressure scale height and the central or midplane density, respectively. In addition I write:

$$
\int_{-\infty}^{\infty} c_{\mathrm{v}} \rho \frac{d T}{d t} d z \sim c_{\mathrm{v}} \Sigma \frac{d T_{\mathrm{c}}}{d t},
$$

where $T_{\mathrm{c}}$ is the central temperature. In accretion discs around white dwarfs radiation pressure is negligible. According to the $\alpha$-prescription the vertical integrated stress is then (see Eq. (4.15)):

$$
\begin{aligned}
T_{r \phi} \equiv \int_{-\infty}^{\infty} t_{r \phi} d z & =2 t_{r \phi, \mathrm{c}} H \\
& =2 \alpha P_{\mathrm{c}} H .
\end{aligned}
$$

For the viscous heating one derives:

$$
\begin{aligned}
Q_{+} & =\eta\left(R \frac{\partial \Omega_{\mathrm{K}}}{\partial R}\right)^{2} \\
& =T_{r \phi} \frac{3}{2} \Omega_{\mathrm{K}} \\
& =v_{\mathrm{c}} \frac{9}{4} \Omega_{\mathrm{K}}^{2} \Sigma
\end{aligned}
$$

where the central kinematic viscosity is given by:

$$
\mathrm{v}_{\mathrm{c}}=\frac{2}{3} \alpha \frac{R_{g} T_{\mathrm{c}}}{\mu \Omega_{\mathrm{K}}} .
$$

Using these approximations we derive from the basic equations (4.6), (4.7), (4.8) and (4.9) the following one dimensional equations:

$$
\frac{\partial \Sigma}{\partial t}=\frac{1}{R} \frac{\partial}{\partial R}\left(R \Sigma v_{R}\right)+\frac{\dot{M}_{\mathrm{tr}}(t, R)}{2 \pi R},
$$




$$
\begin{gathered}
R \frac{\partial\left(\Sigma R^{2} \Omega\right)}{\partial t}=-\frac{\partial}{\partial R}\left(R^{3} \Omega \Sigma v_{R}\right)+\frac{\partial}{\partial R}\left(R^{3} v \Sigma \frac{\partial \Omega}{\partial R}\right) \\
\frac{1}{2}\left(\Sigma c_{\mathrm{v}} \frac{d T}{d t}-\left(\Gamma_{3}-1\right) c_{\mathrm{v}} T \frac{d \Sigma}{d t}\right)=\frac{9 v \Sigma \Omega^{2}}{8}-\frac{H}{R} \frac{\partial\left(R F_{R}\right)}{\partial R}-\sigma T_{\mathrm{eff}}^{4} .
\end{gathered}
$$

Using $\Omega=\Omega_{\mathrm{K}}$ the equations (4.34) and (4.35) can be combined to

$$
\frac{\partial \Sigma}{\partial t}=\frac{3}{R} \frac{\partial}{\partial R}\left(R^{\frac{1}{2}} \frac{\partial}{\partial R}\left(R^{\frac{1}{2}} v \Sigma\right)\right)+\frac{\dot{M}_{\mathrm{tr}}(t, R)}{2 \pi R} .
$$

With the exception of $T_{\text {eff }}$ the equations above contain only mid-plane (or central) variables. By fitting power-laws to the results of the vertical structure calculations, one may obtain the relation between $T_{\text {eff }}$ and the central conditions and, thus, close the system. This has been done by various authors (e.g. Cannizzo 1993b; Ludwig $\&$ Meyer 1998). The resulting power-laws are often called the cooling functions.

\subsection{Simulation of dwarf nova outbursts}

In order to describe the numerical method which I use to solve the differential equations above, I first rewrite the energy equation in a common form (e.g. Cannizzo 1993a):

$$
\frac{\partial T_{\mathrm{c}}}{\partial t}=\frac{2(h-C+J)}{c_{\mathrm{p}} \Sigma}-\frac{R_{\mathrm{g}} T_{\mathrm{c}}}{\mu c_{\mathrm{p}}} \frac{1}{R} \frac{\partial\left(R v_{\mathrm{R}}\right)}{\partial R}-v_{\mathrm{R}} \frac{\partial T_{\mathrm{c}}}{\partial R}
$$

where

$$
v_{\mathrm{R}}=\frac{-3 v}{R} \frac{\partial \log \left(v \Sigma R^{\frac{1}{2}}\right)}{\partial \log R}
$$

is the local radial flow velocity, $h=\frac{9}{8} \mathrm{v} \Omega^{2} \Sigma$ represents viscous heating and $C=$ $\sigma T_{\text {eff }}^{4}$ is the radiative cooling. The frequently used term $J=\frac{H}{R} \frac{\partial F_{R}}{\partial R}$, where $F_{\mathrm{R}}=$ $-\frac{4 a c T^{3}}{3 \kappa_{R} \rho} \frac{\partial T}{\partial R}$ is replaced with

$$
J=\frac{3}{2} c_{\mathrm{p}} \vee \frac{\Sigma}{R} \frac{\partial}{\partial R}\left(R \frac{\partial T}{\partial R}\right)
$$

representing the radial energy carried away by viscous processes. Cannizzo (1993a) showed that the contributions of both terms are roughly of the same order.

Throughout this thesis I use the same boundary conditions as Cannizzo (1993a): at the inner disc radius I use $\Sigma\left(R_{\text {in }}\right)=0$, which allows any material there to be accreted on the central star; at the outer disc radius I adjust the $\Sigma(R)$ profile so that 


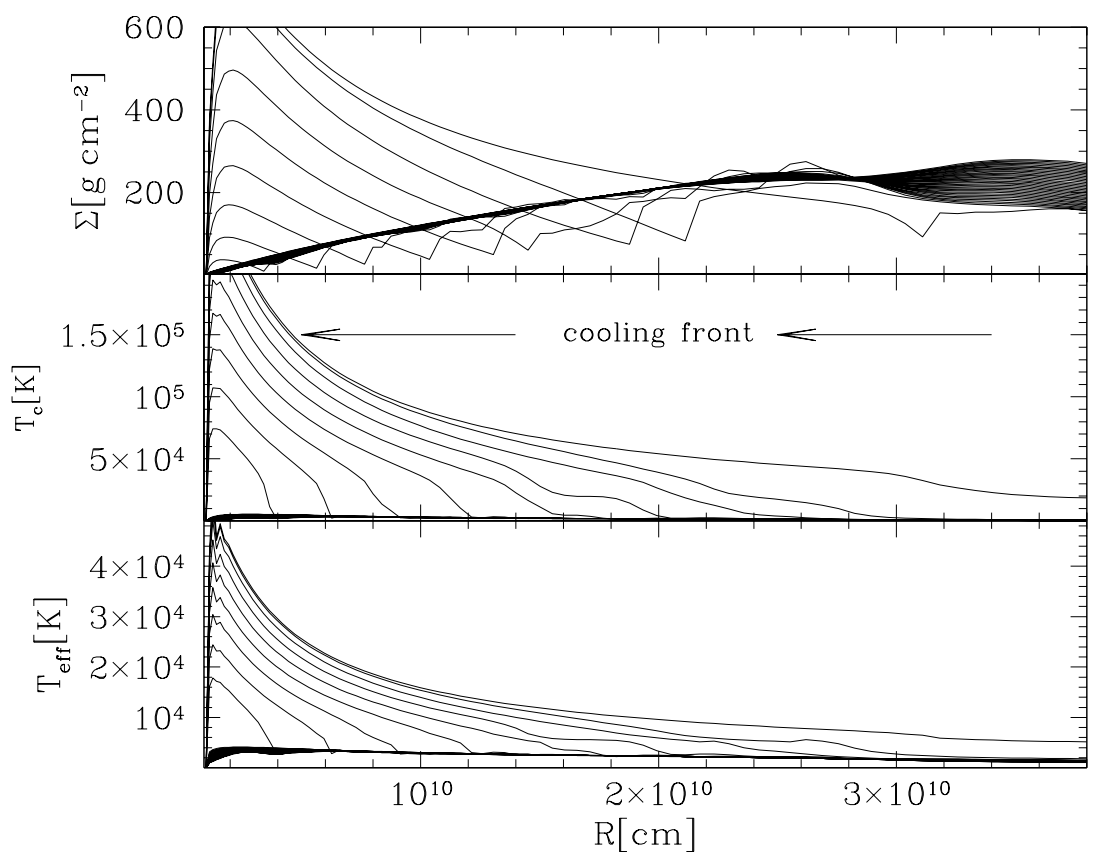

Figure 4.7: The evolution of the disc into quiescence. The lines correspond to the structure of the disc every 4.17 days during the propagation of a cooling front. When the surface density at the outer edge becomes lower than the critical value defined by the S-curve, the outer edge of the disc switches to its equilibrium value at the lower branch. Therefore, the mass transferred to the inner neighbouring annulus decreases until this annulus switches also to the lower branch. This results in a cooling wave moving inward through the disc.

$v_{\mathrm{R}}=0$ which corresponds to having a tidal-like force $F$ of the form $F \propto\left(\frac{R}{R_{\text {out }}}\right)^{\eta}$, where $\eta \rightarrow \infty$.

The Eqs. (4.37) and (4.38) are solved using a combined Finite-Element / FiniteDifference algorithm (FE for the spatial part and FD for the time-evolution). Apart from this work the method of Finite-Elements has not been used in the context of disc accretion onto white dwarfs. As this method proved to be extremely robust, it warrants a somewhat more detailed description.

The idea of FE is to divide the region of interest (the disc radii between $R_{\text {in }}$ and $R_{\text {out }}$ ) into $n-1$ elements and to expand the function $u(x)$, which is supposed to solve the differential equation with suitable functions $u(x)=\sum_{i=1}^{n} a_{i} \varphi_{i}(x)$ for every element. In order to get a continuous solution over all elements, the functions $\left(\varphi_{i}\right)$ of every element have to be transformed to the so-called local basis functions $\left(N_{i}\right)$ and the coefficients to the so-called nodes $\left(c_{i}\right)$ before collected together 


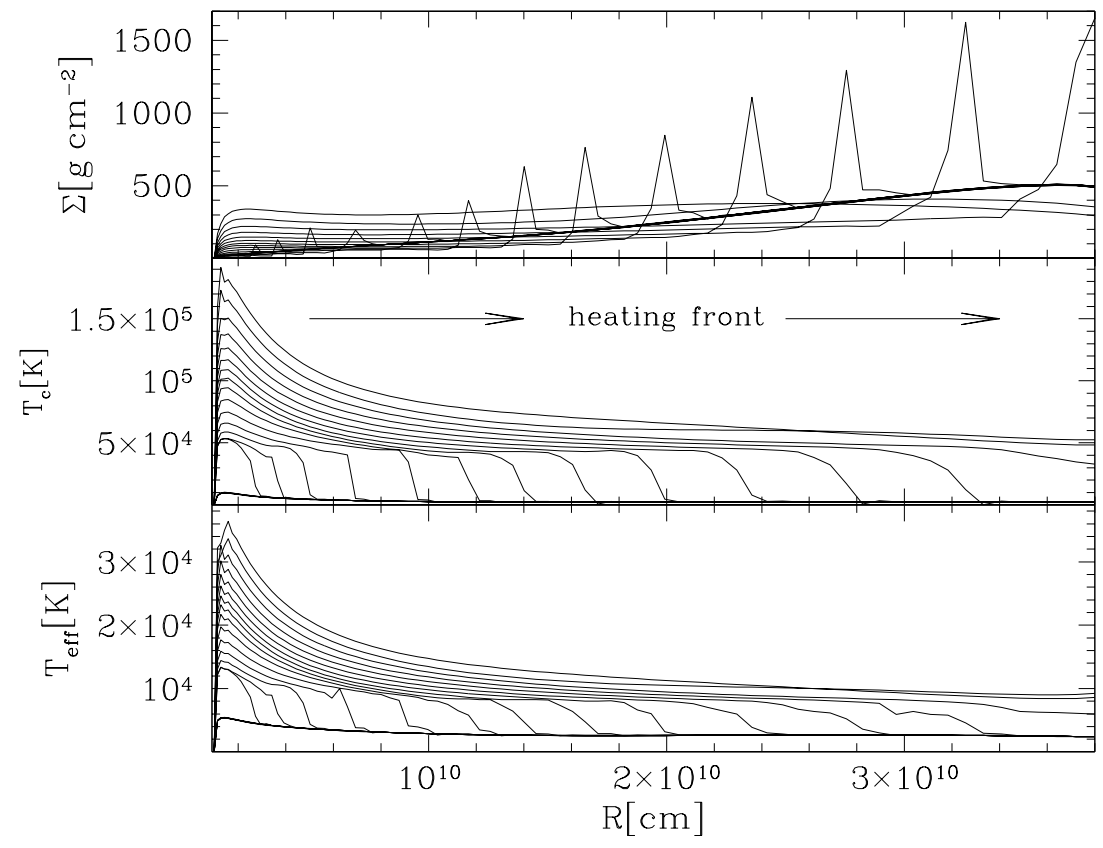

Figure 4.8: The propagation of a heating wave through the disc. The lines correspond to the structure of the disc every 0.46 days during the propagation of a heating front. At the inner edge the surface density reaches the critical value of the lower branch and heats up to thermal equilibrium on the hot upper branch. The increased viscosity leads to increasing surface density of the neighbouring annulus until this annulus also reaches the critical value. This "snowplow-effect" results in a heating wave which transforms the entire disc into the hot state.

(Gruber \& Rappaz 1985, Schwarz 1991). To solve the differential equation the function

$$
u(x)=\sum_{k=1}^{n} c_{i} N_{i}(x)
$$

has to meet the requirement formulated by Galerkin: the integral of the residuum (which one gets by inserting Eq. (4.41) into the differential equation), weighted with the functions $N_{j}(x)(j=1, \ldots, n)$, has to vanish. This requirement, the interchange of integration and summation and partial integration lead to matrixequations of the form

$$
B \dot{c}+A c=D
$$

with $A=\left(a_{i j}\right), B=\left(b_{i j}\right)$ and $D=\left(d_{i}\right)(i=1, . ., n ; j=1, . ., n$ for $n$ nodes $)$.

To solve the differential Eqs. (4.37) and (4.38), I have to fill $A, B, D$ in the sense mentioned above and calculate $c$ from Eq. (4.42). After transforming to the vari- 


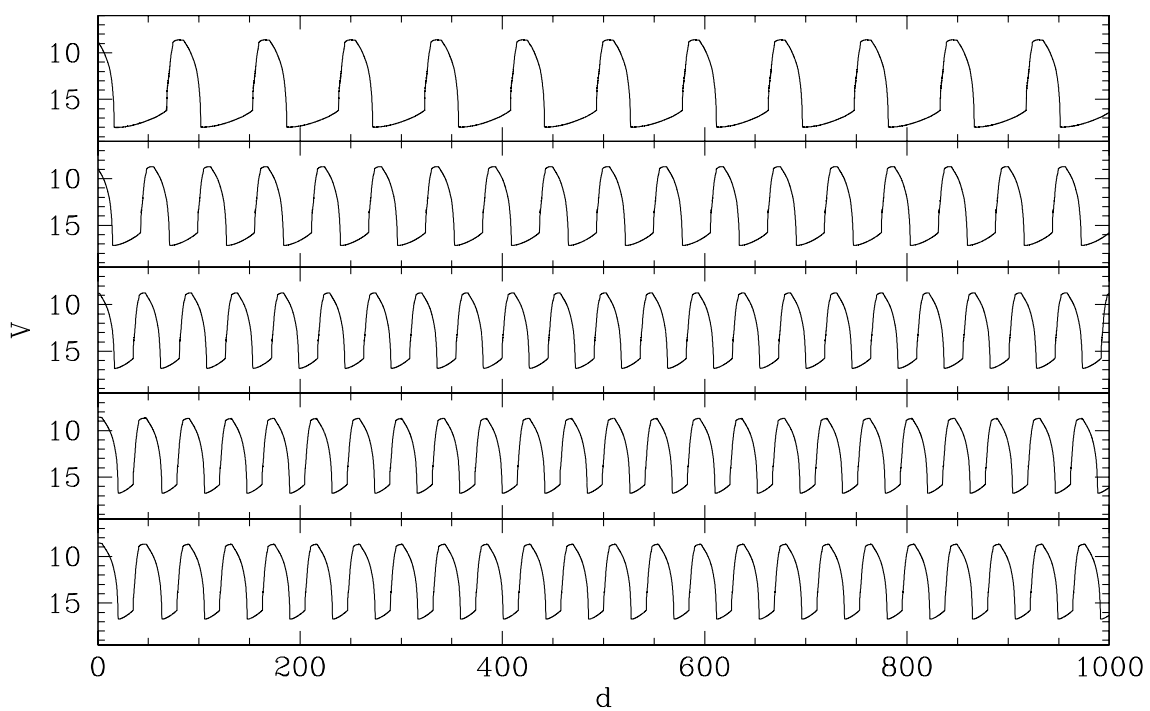

Figure 4.9: Comparison of the resulting light curves with those calculated by Cannizzo (1993b, his Fig.6d). From top to bottom I used 40, 60, 80, 100 and 200 nodes. Convergence is obtained for 100 nodes.

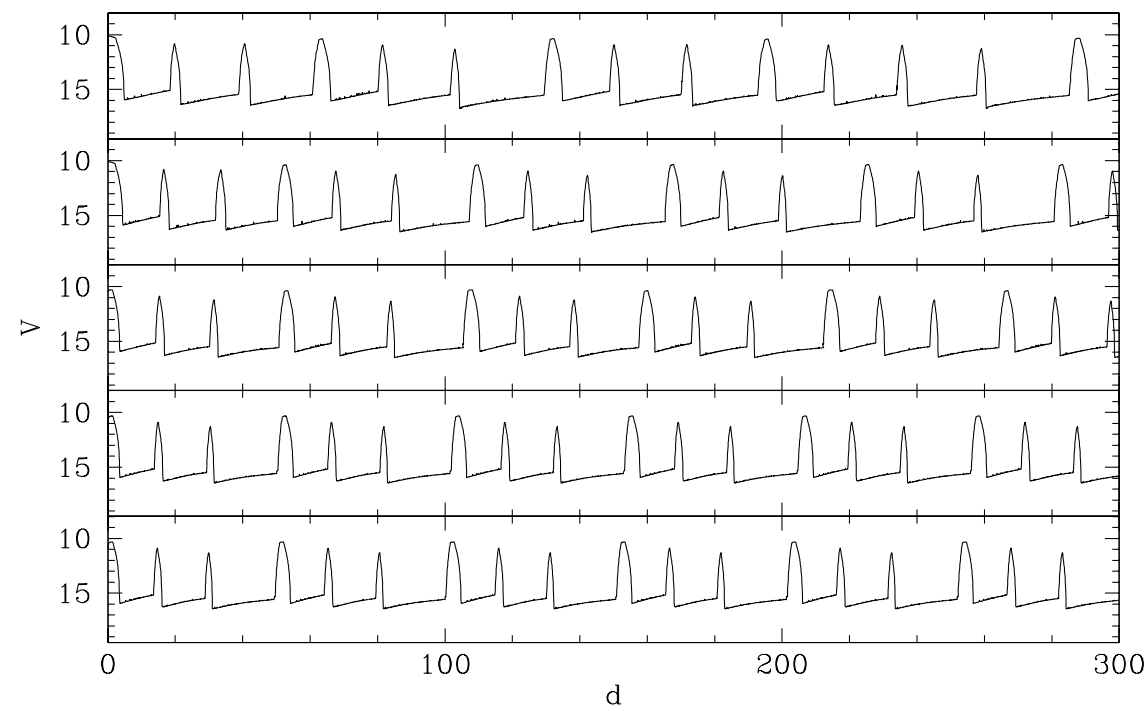

Figure 4.10: Comparison of the resulting light curves with those calculated by Ludwig \& Meyer (1998). From top to bottom I used again 40, 60, 80, 100 and 200 nodes. My code accurately reproduces the sequence of one long outburst followed by two short outbursts. 
ables $X=2 R^{\frac{1}{2}}$ and $S=X \sum$ and using Eq. (4.37), I derive for the surface density:

$$
\begin{aligned}
a_{i j}= & \sum_{k=1}^{n} v_{k}\left(\int \frac{12}{X^{2}} \frac{\partial N_{i}}{\partial X} N_{k} \frac{\partial N_{j}}{\partial X} d X\right. \\
& \left.+\int \frac{12}{X^{2}} N_{i} \frac{\partial N_{k}}{\partial X} \frac{\partial N_{j}}{\partial X} d X\right), \\
b_{i j}= & \int N_{i} N_{j} d X \\
d_{i}= & \sum_{k=1}^{n} \int \frac{2 \dot{M}_{\mathrm{tr}, k} N_{k}}{\pi X^{2}} N_{j} d X .
\end{aligned}
$$

Similarly, it is easy to obtain the following equations for the central temperature from Eq. (4.38):

$$
\begin{aligned}
a_{i j}= & \sum_{k=1}^{n}\left(\int p_{k}^{(1)} \frac{\partial N_{i}}{\partial R} N_{k} \frac{\partial N_{j}}{\partial R} d R\right. \\
& \left.+\int p_{k}^{(2)} N_{k} \frac{\partial N_{i}}{\partial R} N_{j} d R+\int p_{k}^{(3)} N_{i} N_{k} N_{j} d R\right), \\
b_{i j}= & \int N_{i} N_{j} d R \\
d_{j}= & \sum_{k=1}^{n} \int p_{k}^{(4)} N_{k} N_{j} d R .
\end{aligned}
$$

The coefficients $p_{k}^{(i)}$ are given by

$$
\begin{aligned}
p_{k}^{(1)} & =3 c_{\mathrm{p}} v \Sigma, \\
p_{k}^{(2)} & =\frac{3 c_{\mathrm{p}} v \Sigma}{R}-v_{\mathrm{R}}, \\
p_{k}^{(3)} & =\frac{R_{\mathrm{g}}}{\mu c_{\mathrm{p}} R} \frac{\partial R v_{\mathrm{R}}}{\partial R}, \\
p_{k}^{(4)} & =\frac{9 / 4 v \Sigma \Omega^{2}-\sigma T_{\mathrm{eff}}}{c_{\mathrm{p}} \Sigma} .
\end{aligned}
$$

The index $k$ refers to the value of the quantities at node number $k$ which is equivalent to the radius $R_{k}$. Notice that I only used linear basis functions throughout this thesis.

As mentioned above, Eq. (4.42) has to be solved to get $c(t+\Delta t)$ from $c(t)$. Using simple finite differences leads to

$$
\begin{aligned}
c(t+\Delta t)= & c(t) \frac{1}{2} \Delta t\left(B^{-1} A c(t)\right. \\
& \left.+B^{-1} A c(t+\Delta t)\left(+B^{-1} D\right)\right) .
\end{aligned}
$$


In order to test my code, I carried out two sets of calculations using the binary parameters and cooling functions from Cannizzo (1993a)

$$
\begin{aligned}
& M_{1}=1 M_{\odot}, \alpha_{\mathrm{h}}=0.1, \alpha_{\mathrm{c}}=0.02, \\
& R_{\mathrm{in}}=5.0 \times 10^{8} \mathrm{~cm}, R_{\mathrm{out}}=4.0 \times 10^{10} \mathrm{~cm}, \\
& \dot{M}_{\mathrm{tr}}=1.5 \times 10^{9} M_{\odot} / \mathrm{yr}
\end{aligned}
$$

and Ludwig \& Meyer (1998)

$$
\begin{aligned}
& M_{1}=0.6 M_{\odot}, \alpha_{h}=0.2, \alpha_{c}=0.04, \\
& R_{\text {in }}=8.4 \times 10^{8} \mathrm{~cm}, R_{\text {out }}=1.7 \times 10^{10} \mathrm{~cm}, \\
& \dot{M}_{\mathrm{tr}}=5 \times 10^{15} \mathrm{~g} / \mathrm{s} .
\end{aligned}
$$

The resulting light curves are shown in Figs. 4.9 and 4.10. My code reproduces the sequence of only relatively long outbursts found by Cannizzo (1993b) for the parameters of SS Cygni as well as the sequence of one long outburst followed by two short outbursts found by Ludwig \& Meyer (1998) to describe VW Hydri. The short outbursts arise when there is not enough mass stored in the disc and therefore the heating wave gets reflected before it has reached the outer edge of the disc.

I find that at least 100 nodes are necessary for long-term convergence. The outburst and quiescence duration decreases with an increasing number of nodes because - with finer zoning - the amount of time spent on the viscous plateau becomes shorter (Cannizzo 1993b). This effect is smaller in Fig. 4.10 because the disc in VW Hydri is smaller than that in SS Cygni.

I conclude that my FE-code produces results which are in excellent agreement with those of other fine-mesh computations.

\subsection{Confrontation with observation}

The time-dependent equations for mass and energy transfer through the disc have been integrated for a wide range of prescriptions for the crucial parameter $\alpha$. For $\alpha=$ const Smak (1984) finds low amplitude outbursts only. In general, in order to achieve realistic outbursts it has been found necessary to adopt a larger $\alpha$ in the hot than in the cool state. Typical values for the viscosity parameter are $\alpha_{\mathrm{h}} \sim 0.1$ and $\alpha_{c} \sim 0.02$, which I also used in the previous section.

The limit-cycle model has become the generally accepted explanation for dwarf nova outbursts observed in many CVs. The only competing model is the mass transfer instability model, in which the mass transfer from the secondary switches between two states. Although it is interesting to compare the two models it is 
probably fair to say there is nowadays very few support for the mass transfer instability model. The reasons giving preference for the disc limit cycle model may be seen in theoretical as well as in observational constraints:

- The simplest argument comes from the fact that the magnetic systems which do not have accretion discs show no outbursts. This indicates that the accretion disc is the origin of the outbursts. There is no reason to expect the secondaries in AMHer systems and those in dwarf nova systems to differ, which would be necessary if the mass transfer instability model were correct.

- The bright spot maintains a nearly constant luminosity during the onset of an outburst, which contradicts the predictions of the mass transfer instability model.

- The critical accretion rate at the outer edge of the disc defines a dividing line between persistent (nova-like) CVs and dwarf nova systems. This theoretical dividing line is independent of the crucial parameter $\alpha$ and consistent with observations (Warner 1995). The mass transfer instability model is not able to explain the different behaviour of nova-like and dwarf nova systems.

- The disc limit-cycle model is also consistent with the dispersion in the rise time of an outburst and the fact that the decay time is always about the same. In the disc limit-cycle model the heating front can start at each annulus whereas the cooling wave always begins at the outer edge of the disc.

- The observed concave downward decay shape of dwarf nova light curves is consistent with the disc limit-cycle model and contrasts with the exponential decay predicted by the mass transfer instability model.

Although the support for the disc limit-cycle model is strong, it faces several difficulties:

- The disc instability model in the common form predicts strictly periodic outbursts while the observed cycles are only approximately regular and have varying outburst magnitudes (e.g. Cannizzo \& Mattei 1992).

- To explain the superoutbursts of the SU UMa type systems an additional ingredient is required (thermal-tidal instability (Osaki 1989); enhanced mass transfer during outburst (Smak 1996)). 
- Although most dwarf nova systems require values for $\alpha$ of 0.1 for the hot and 0.02 for the cold state, the long recurrence times of the WZ Sge type dwarf nova outbursts are only reproduced by the disc instability model using a very low value, i.e. $\alpha \sim 10^{-3}$ during quiescence.

- In general, using the ad hoc $\alpha$-prescription for the viscosity is not satisfactory.

In addition to the partial disagreement with the observations and the uncertainties in $\alpha$, the disc limit-cycle model in the basic form which I presented above, neglects important boundary conditions imposed by the environment on the disc. In the next three chapters I examine in detail: (1) the influence which variations of the mass transfer rate from the secondary have on the outburst behaviour; (2) what happens if the matter arriving from the secondary partially overflows the outer disc; (3) how the dynamical behaviour and the structure of the disc are influenced by irradiation of the white dwarf. 


\section{Chapter 5}

\section{AM Her as a dwarf nova}

In the previous section I have rewritten the equations describing the evolution of viscous discs and explained how I solved them. This section focuses on the interaction between the evolution of the disc and variations of the mass transfer rate. The rate at which mass is transferred from the secondary to the accretion disc is an important boundary condition of the disc limit-cycle model. In most studies of dwarf nova outbursts this mass transfer rate is kept constant (Cannizzo 1993a; Ludwig \& Meyer 1998; Hameury et al. 1998, 1999). Duschl \& Livio (1989) were the first to examine combined mass transfer and disc outbursts, though within the framework of the mass transfer instability model where individual and short-lived mass transfer events are capable of producing single outbursts. Smak $(1991,1999)$ discussed mass transfer variations in the context of the superoutburst phenomenon in dwarf novae of the SU UMa type and dwarf nova outbursts with enhanced mass transfer during outburst.

King \& Cannizzo (1998) and Leach et al. (1999) tested how the accretion disc in a dwarf nova system behaves if the mass transfer from the secondary varies abruptly between different levels.

They found that these mass transfer variations produce only subtle effects on normal dwarf novae, including variations in the outburst shape, and that the dwarf novae keep on having outbursts even if the transfer rate is reduced close to zero.

In spite of these efforts we are left with the question of what the real variations of the mass transfer rates from the secondaries in dwarf nova systems are, how they can influence the outburst behaviour of the accretion discs and if they can account for the observed departures from strictly periodic light curves. Fortunately, nature provides an answer to the first question in the form of discless cataclysmic variables, the polars or AM Her systems. In these systems (see chapter 2), the mass transfer rate can be estimated directly from the observations because there is no 
accretion disc acting as a mass buffer. Thus, I can use the long-term light curve of an AMHer system as a measure for the mass transfer variations in a fictitious but realistic dwarf nova system with the same system parameters. In this chapter, I present the results of such a numerical experiment.

In the next section I discuss briefly various types of explanations for the "observed" variations of the mass transfer rate in magnetic systems. Than I describe how to derive the mass transfer rate in AM Her as a function of time, $\dot{M}_{\mathrm{tr}}(t)$ and thereafter I apply this mass transfer rate to a fictitious dwarf nova and discuss the effects that can be observed in the outburst behaviour.

\subsection{The cause of mass transfer variations}

Magnetic CVs show irregular variations of their visual brightness with the changes occurring on time scales ranging from days to months. As the magnetic systems do not have discs, the origin of the phenomenon is unambiguously placed on the secondary star. Ritter (1988) finds that the mass transfer rate depends on the photospheric scale height of the secondary $H_{\mathrm{sec}}$ and the difference between the Roche lobe radius and the secondaries radius $\Delta R=R_{\mathrm{L}}-R_{\mathrm{sec}}$. He derived:

$$
-\dot{M}_{\mathrm{tr}}=\dot{M}_{\mathrm{tr}, 0} e^{\frac{\Delta R}{H_{\mathrm{sec}}}}
$$

where $H_{\mathrm{sec}}=k T R_{\mathrm{sec}}^{2} / G M_{\mathrm{sec}}$ with $k$ the Boltzman constant and $T$ the surface temperature of the secondary. Mass transfer variations must result from variations of $\frac{\Delta R}{H_{\text {sec }}}$. Several hypothesis have been discussed during the last decade. A decrease of $\Delta R$, for example, due to increasing $R_{\mathrm{L}}$ caused by injecting angular momentum from dipole-dipole interactions (e.g. King et al. 1990) is found to be rather inefficient. Another possibility is a decrease of $H_{\mathrm{sec}}$ resulting from occasionally vanishing irradiation of the secondary, but it is not clear how the system can make sporadic transitions between the irradiated and the non irradiated case (King \& Cannizzo 1998).

It is now widely accepted that starspots, which lead to a decreased value of $H_{\mathrm{sec}}$ are the cause of the "observed" mass transfer variations in magnetic CVs. As the strong magnetic pressure in a spot reduces the gas pressure and hence, the temperature, it leads to a decrease of $H_{\mathrm{sec}}$. This effect is most recently discussed by Livio \& Pringle (1994). Spots may grow near the $L_{1}$ point or form elsewhere and drift across it, leading to a decrease of $H_{\text {sec }}$ which results in a decrease of $\dot{M}_{\text {tr }}$ (see Eq. (5.1)). Indeed, Hessman et al. (2000) derive spot distributions for the secondary in AM Her using the mass transfer history of the system.

As starspots are the most likely explanation for these mass transfer variations in 

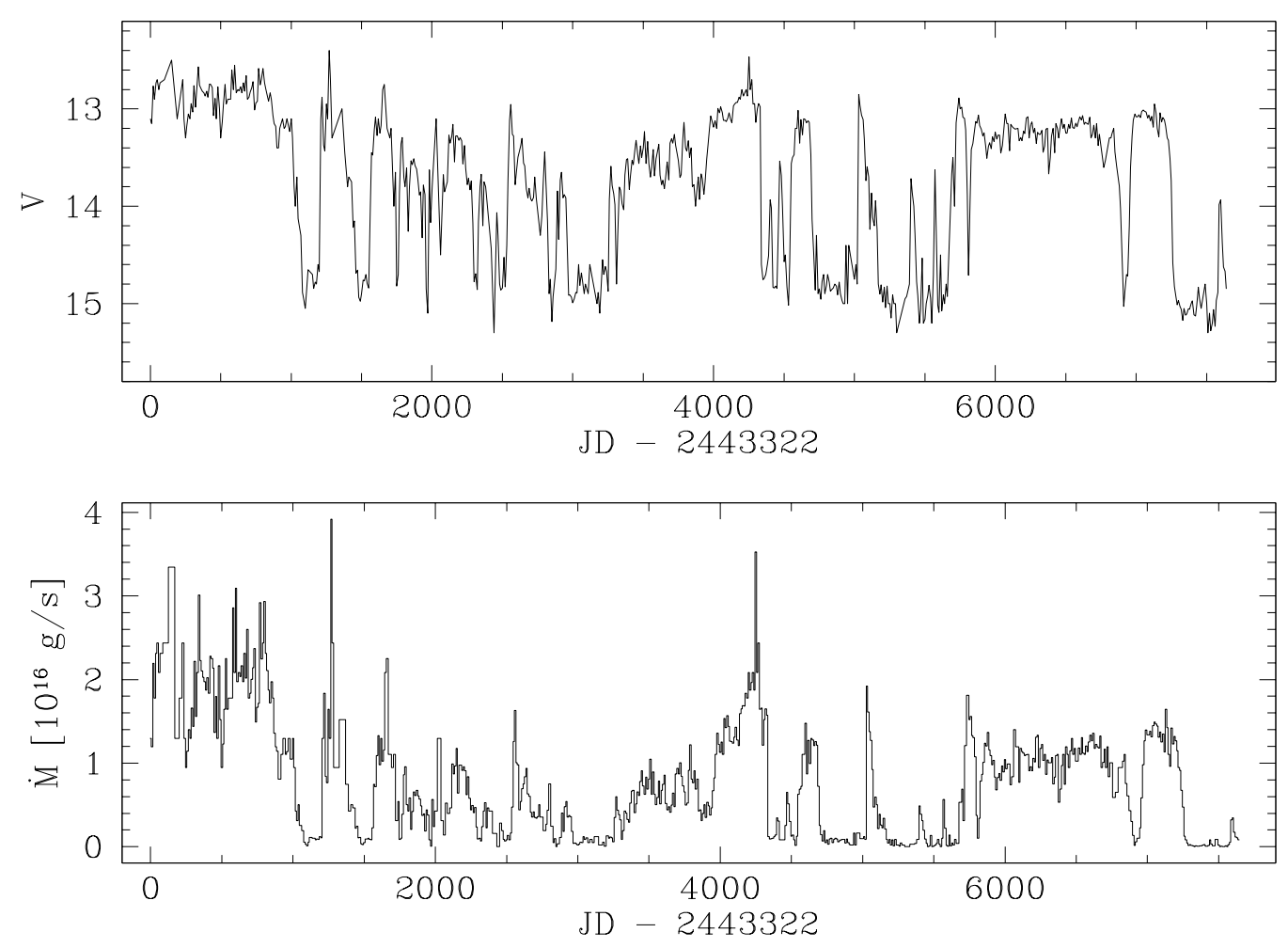

Figure 5.1: The light curve and the derived mass transfer rate in AM Her as a function of time.

magnetic systems, there is no reason for these variations not being present in non magnetic CVs.

\subsection{The mass loss rate of the secondary star in AM Herculis}

As noted above, the strong magnetic field of the white dwarf primary in polars prevents the formation of an accretion disc. Without an accretion disc acting as a buffer for the transferred mass, the mass loss rate from the secondary equals the mass accretion rate on the white dwarf at every moment, $\dot{M}_{\text {acc }}=\dot{M}_{\text {tr }}$ (the free-fall time is $\leq 1 \mathrm{~h}$ ). As an observational consequence, any variation in the rate at which the secondary star loses mass through the $L_{1}$ point will result in a quasi-immediate change of the observed accretion luminosity. The brightest polar, AMHer, has 
been intensively monitored at optical wavelengths by observers of the AAVSO (American Association of Variable Star Observers) for more than 20 years and shows an irregular long-term variability, switching back and forth between highand low states of accretion on timescales of days to months. The light curve of AM Her is shown in the upper panel of Fig. 5.1.

The problem of deriving the mass loss history of the secondary star is then equivalent to that of determining the accretion luminosity $L_{\text {acc }}$ as a function of time. As the bulk of the accretion luminosity is emitted in the X-ray regime, a bolometric correction relating the densely monitored optical magnitude to the total luminosity has to be derived. This approach has been followed in detail by Hessman et al. (2000) using X-ray observations obtained at multiple epochs. I summarise only briefly the results here. The accretion luminosity is computed from the observed accretion-induced flux $F_{\text {acc }}$ as

$$
L_{\mathrm{acc}}(t)=4 \pi d^{2} F_{\mathrm{acc}}(t)
$$

with $d=90 \mathrm{pc}$ (Gänsicke et al. 1995). It is further used $F_{\mathrm{acc}} \approx F_{\mathrm{SX}}+3 \times F_{\mathrm{HX}}$ with $F_{\mathrm{SX}}$ and $F_{\mathrm{HX}}$ the observed soft and hard X-ray fluxes respectively. The factor three accounts for the additional cyclotron radiation emitted from the accretion column and for the thermal reprocession of bremsstrahlung and cyclotron radiation intercepted by the white dwarf and emitted in the ultraviolet (Gänsicke et al. 1995). The mass loss ( $=$ transfer) rate is then

$$
\dot{M}_{\mathrm{tr}}(t)=\frac{L_{\mathrm{acc}}(t) R_{\mathrm{wd}}}{G M_{\mathrm{wd}}}
$$

where $G$ is the gravitational constant and $R_{\mathrm{wd}}$ and $M_{\mathrm{wd}}$ are the white dwarf radius and mass, respectively. As the actual properties of the white dwarf in AMHer are still the subject of controversial discussions (Gänsicke et al. 1998a; Cropper et al. 1999), I use the parameters of an average white dwarf, $M_{\mathrm{wd}}=0.6 M_{\odot}$ and $R_{\mathrm{wd}}=$ $8.4 \times 10^{8} \mathrm{~cm} . \dot{M}_{\mathrm{tr}}(t)$ is shown in Fig. 5.1. The average value of the mass transfer rate in AMHer is $\dot{M}_{\mathrm{av}}=7.88 \times 10^{15} \mathrm{~g} \mathrm{~s}^{-1}=1.24 \times 10^{-10} M_{\odot} \mathrm{yr}^{-1}$. The derived mass transfer rates of AM Her are in general agreement with results published in the literature. For example, Beuermann \& Burwitz (1995) found transfer rates between 0.8 and $2.0 \times 10^{-10} M_{\odot} \mathrm{yr}^{-1}$ and Greeley et al. (1999) estimated a mass transfer rate of $2 \times 10^{16} \mathrm{~g} \mathrm{~s}^{-1}$ for the high state of AMHer from far ultraviolet spectra. 


\subsection{Results}

\subsubsection{The fictitious dwarf nova}

I devised a fictitious dwarf nova with a non-magnetic primary of mass $M_{\mathrm{wd}}=$ $0.6 M_{\odot}$ and an orbital period of $P=3.08 \mathrm{hr}$, i.e. a non-magnetic twin of AMHer. For these binary parameters, I obtain $R_{\text {in }} \simeq R_{\mathrm{wd}}=8.4 \times 10^{8} \mathrm{~cm}$ for the inner disc radius and $R_{\text {out }}=2.2 \times 10^{10} \mathrm{~cm}$ for the outer edge of the disc. I then used my FE code to follow the structure of the accretion disc in my fictitious dwarf nova for $7000 \mathrm{~d}$, applying the variable mass transfer rate $\dot{M}_{\mathrm{tr}}(t)$ derived above and standard viscosity parameters $\alpha_{h}=0.2$ and $\alpha_{c}=0.04$.

I show 500 day-long samples of my calculations in Figs. 5.2-5.6. In each figure, the top panel show the mass transfer rate as a function of time (solid line) and the average mass transfer rate $\log \left(\dot{M}_{\mathrm{av}}\left[\mathrm{gs}^{-1}\right]\right)=15.90$ (dotted line). The panel below displays the disc mass $M_{\text {disc }}$ normalised with the averaged disc mass $\bar{M}_{\text {disc }}=1.64 \times 10^{23} \mathrm{~g}$. The two lower panels display the light curves calculated with the varying mass transfer rate and the light curves calculated with the constant average mass transfer rate, respectively. For the constant average mass transfer rate the disc goes through a $\sim 60$ day-long cycle including one long outburst followed by two short outbursts. The long outbursts are those in which the entire disc is transformed into the hot state while the short outbursts arise when the outward moving heating wave is reflected as a cooling wave before it has reached the outer edge of the disc.

The numerical experiment clearly demonstrates that the outburst light curve of the fictitious system is strongly affected by the variations of the mass transfer rate. Even in the case of relatively small fluctuations $\left(16.0<\log \left(\dot{M}_{\mathrm{tr}}\left[\mathrm{g} \mathrm{s}^{-1}\right]\right)<\right.$ 16.4) effects on the outburst behaviour are clearly present in the light curve. In Fig. 5.2, the mass transfer rate is always high during the 500 days but the disc switches between an accretion state with only long outbursts and states where one or two short outbursts follow a long outburst. When the transfer rate decreases somewhat ( $\sim$ day 200), the disc does not save enough mass to create consecutive long outbursts until the mass transfer rate increases again ( $\sim$ day 350$)$.

In addition to this effect, my experiment shows that a sharp decrease in the mass transfer rate instantaneously changes the outburst behaviour of the accretion disc. In Fig. 5.3 the disc first behaves as in Fig. 5.2 but when the transfer rate drops sharply (day 4340), the long outbursts immediately vanish and the duration of the quiescent phase increases somewhat.

Another remarkable point is that even during relatively long periods of very low transfer rates (Fig. 5.4, days 4700-5000) the disc does not stop its outburst ac- 


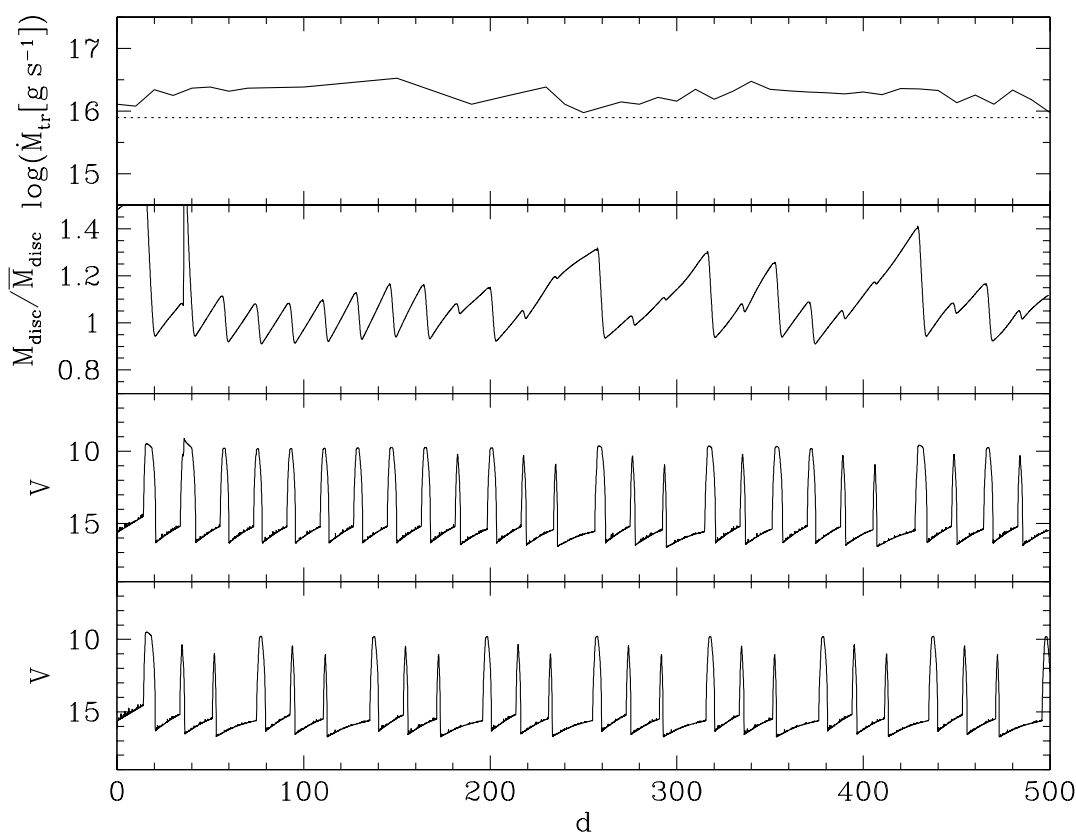

Figure 5.2: From top to bottom as a function of time: the mass transfer rate (top, solid line), the averaged mass transfer rate $\left(\log \left(\dot{M}_{\text {tr }}\right)=15.9\right.$, top, dotted line), the normalised disc mass and the light curves produced by the fictitious dwarf nova with the variable transfer rate and the averaged transfer rate adopted from AM Her.

tivity but produces only short outbursts with slowly decreasing amplitudes and increasing quiescence intervals. This confirms the findings of King \& Cannizzo (1998).

Fig. 5.5 shows 500 days of my simulation in which the adopted mass transfer from the secondary varies strongly on a timescale of roughly 20 days. This is the most frequent case during my calculation but there are rare periods of nearly constant mass transfer. Fig. 5.6 shows the light curve of the fictitious system during 500 days in which the mass transfer rate nearly exactly equals its averaged value (top). As a result the light curves computed with the real mass transfer rate and the averaged value look equal.

In summary, one can say that the variations of the mass transfer rate leads the disc to switch between three states in which only long outbursts occur $\left(\log \left(\dot{M}_{\text {tr }}\right) \geq\right.$ $16.3)$, one long outburst is followed by one or two short outbursts $\left(16.3>\log \left(\dot{M}_{\text {tr }}\right)>\right.$ $15.7)$, and only short outbursts occur $\left(\log \left(\dot{M}_{\text {tr }}\right) \leq 15.7\right)$.

In order to understand the described behaviour of the disc I take into account the viscous timescale $t_{\mathrm{v}} \sim R^{2} / \mathrm{v}$ which gives an estimate of the timescale for a disc annulus to move a radial distance $R$. For the quiescent state $t_{\mathrm{v}, \mathrm{c}}$ and the outburst 


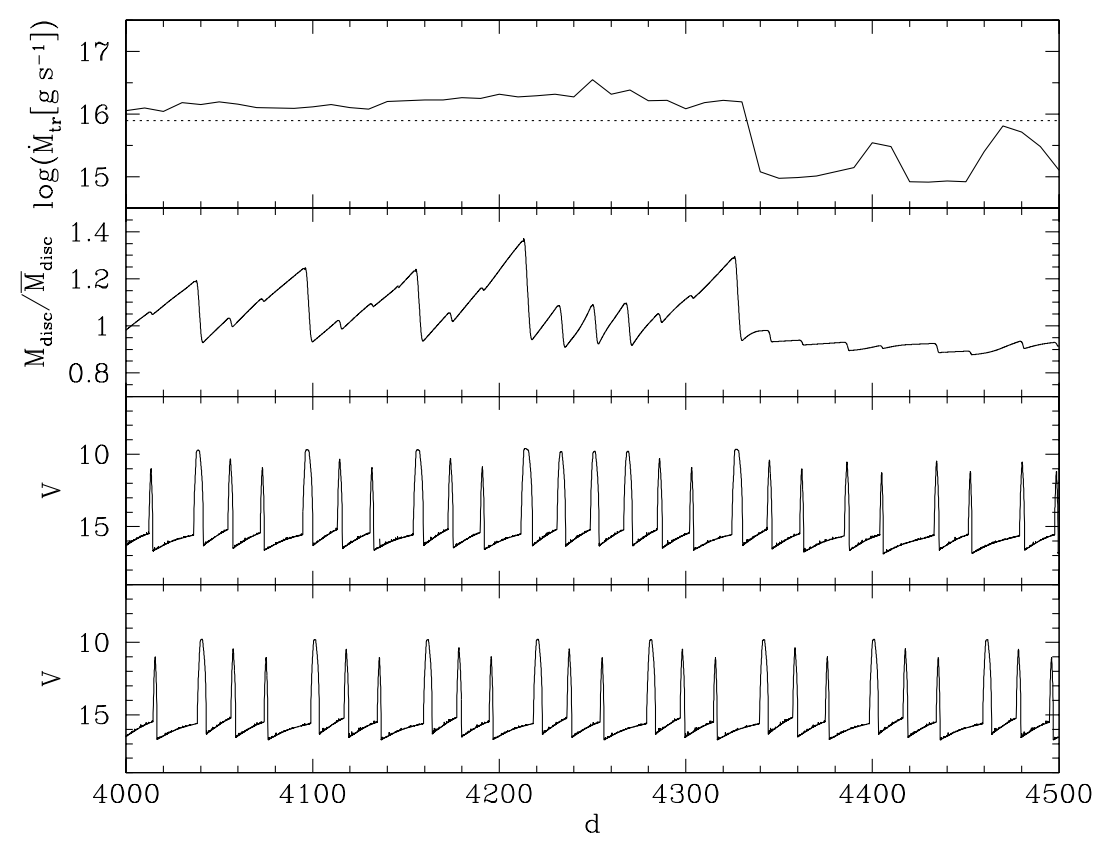

Figure 5.3: The same as Fig. 5.2 but another snapshot of the simulation. The sharp decline of the mass transfer rate is immediately reproduced by the accretion disc.

state $t_{\mathrm{v}, \mathrm{h}}$ and with $R=R_{\text {out }}$ (where the mass transferred from the secondary is added to the disc) I obtain for the viscous timescale

$$
\begin{aligned}
t_{\mathrm{v}, \mathrm{c}} & \sim \frac{R^{2}}{\mathrm{v}} \sim 2000 \mathrm{~d}, \\
t_{\mathrm{v}, \mathrm{h}} & \sim \frac{R^{2}}{\mathrm{v}} \sim 15 \mathrm{~d} .
\end{aligned}
$$

The mass added to the disc during quiescence is stored in the disc because it moves inward on the long timescale $t_{\mathrm{v}, \mathrm{c}}$ whereas during an outburst even mass from outer regions can reach the white dwarf within a viscous timescale. This makes it possible that the mass accreted onto the white dwarf during a long outburst can be up to roughly one third of the disc mass ( $\sim$ day 4220). Therefore the disc can relax to equilibrium with the mass transfer rate in only one outburst in the case of high transfer rates (Fig. 5.2).

The prompt response of the disc to the sharp decline in the mass transfer rate (Fig. 5.3) can be understood in the same way: due to the short viscous time $\left(t_{\mathrm{v}, \mathrm{h}}\right)$ the white dwarf accretes a substantial fraction of the disc mass $\left(\sim 1 / 4 M_{\text {disc }}\right)$ during the last long outburst which immediately prevents long outbursts when the mass transfer rate becomes low ( $\sim$ day 4340). 


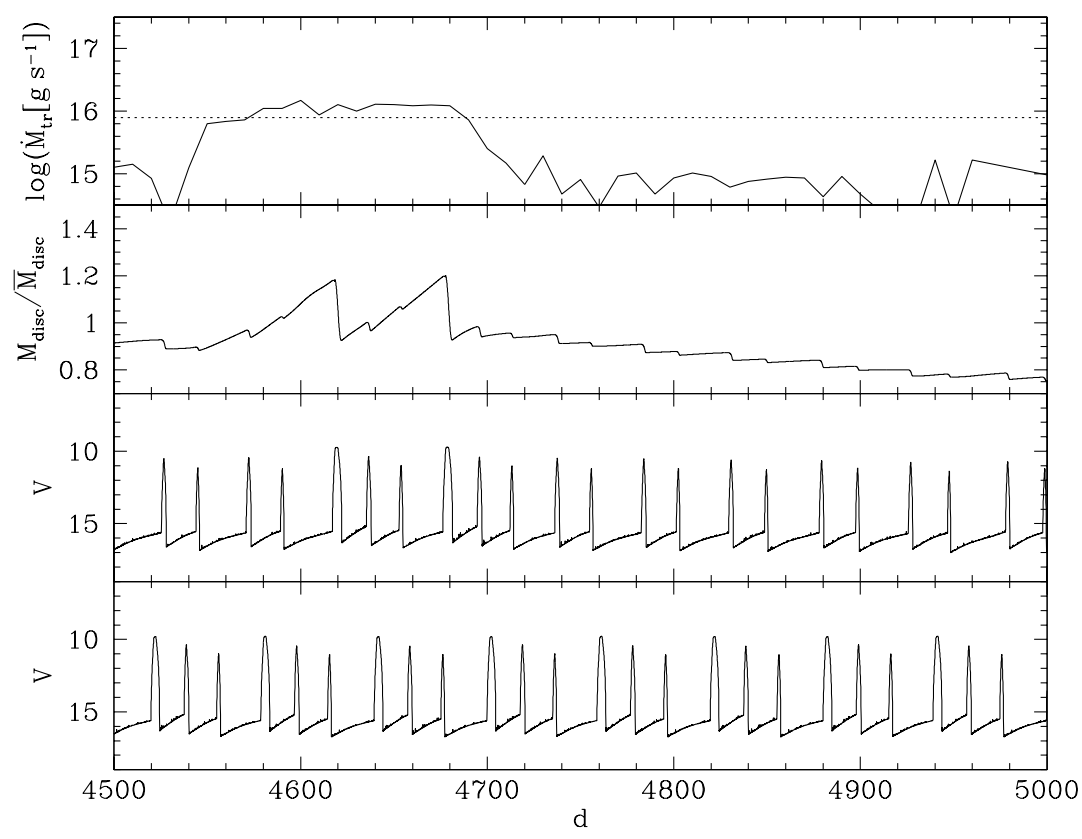

Figure 5.4: The same as Fig. 5.2 but another snapshot of the simulation. Even the long period of low transfer rates does not stop the outburst activity of the disc.

Finally, the long period of low transfer rates (Fig. 5.4) does not prevent outbursts because the mass accreted during the short outbursts is only a few percent of the disc mass. Therefore the disc relaxes to the mass transfer rate on a longer timescale.

\subsubsection{The mass transfer and mass accretion rates}

An important point in understanding the physics of accreting binaries is to know how far the outburst behaviour and hence the resulting light curves depend on real variations of the mass transfer rate.

To answer this question, I compare the averaged mass accretion rate onto the white dwarf with the mass transfer rate. Figure 5.7 shows that the time in which the disc relaxes to an equilibrium with the mass transfer rate depends on the occurrence of long outbursts: when the accretion rate is averaged over 20 days (dotted line in Fig. 5.7) the mass transfer and the accretion rate correspond roughly only during periods where only long outbursts occur (days 40 - 150, see also Fig. 5.2) but for the periods where the disc goes through a cycle of short and long outbursts the accretion rate has to be averaged over 60 days to match the mass transfer rate 


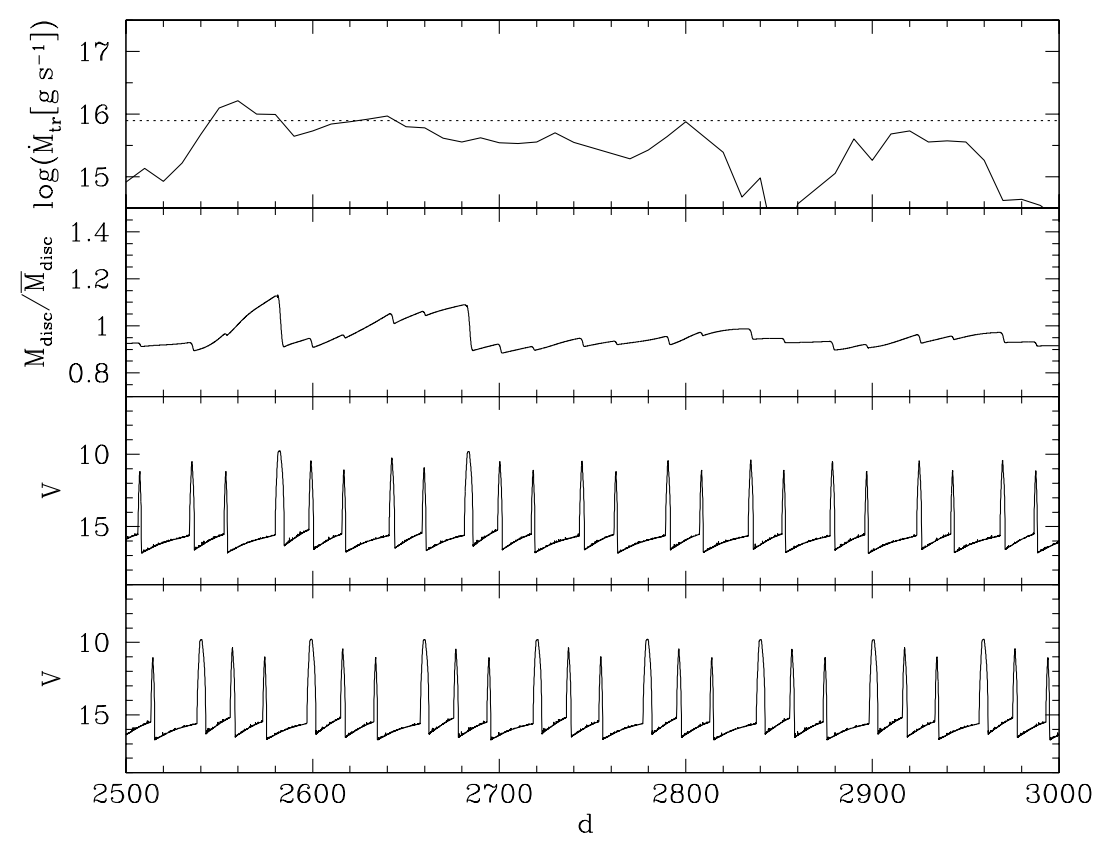

Figure 5.5: The same as Fig. 5.2 but a snapshot of the simulation where the transfer rate strongly varies.

(days 150-500). If the mass transfer rate drops steeply and stays in a low-state (days 4700-5000 in Fig. 10, see also Fig. 5.4), the accretion rate needs more than 60 days to follow this behaviour, because the disc produces only short outbursts in which only a small percentage of the disc mass is involved.

In order to make this plausible I give a relaxation timescale $t_{\mathrm{r}}$ as the ratio of the viscous timescale in outburst with the relative mass fraction accreted during an outburst:

$$
t_{\mathrm{r}} \sim t_{\mathrm{v}, \mathrm{h}} \frac{M_{\mathrm{disc}}}{\Delta M_{\mathrm{disc}}}
$$

For high mass transfer rates this timescale is around 70 days and so the correspondence of the averaged accretion rate and the mass transfer rate in Fig. 5.7. is not surprising. In the case of low transfer rates (Fig. 5.8, day 4700-5000) this timescale is longer ( $\sim 300$ days) because only roughly 5 percent of the disc mass are accreted during an outburst.

\subsubsection{Dependence on the primary mass}

In the numerical experiment above, I have assumed an average white dwarf mass for the primary in AMHer. The literature holds a large spectrum of white dwarf 


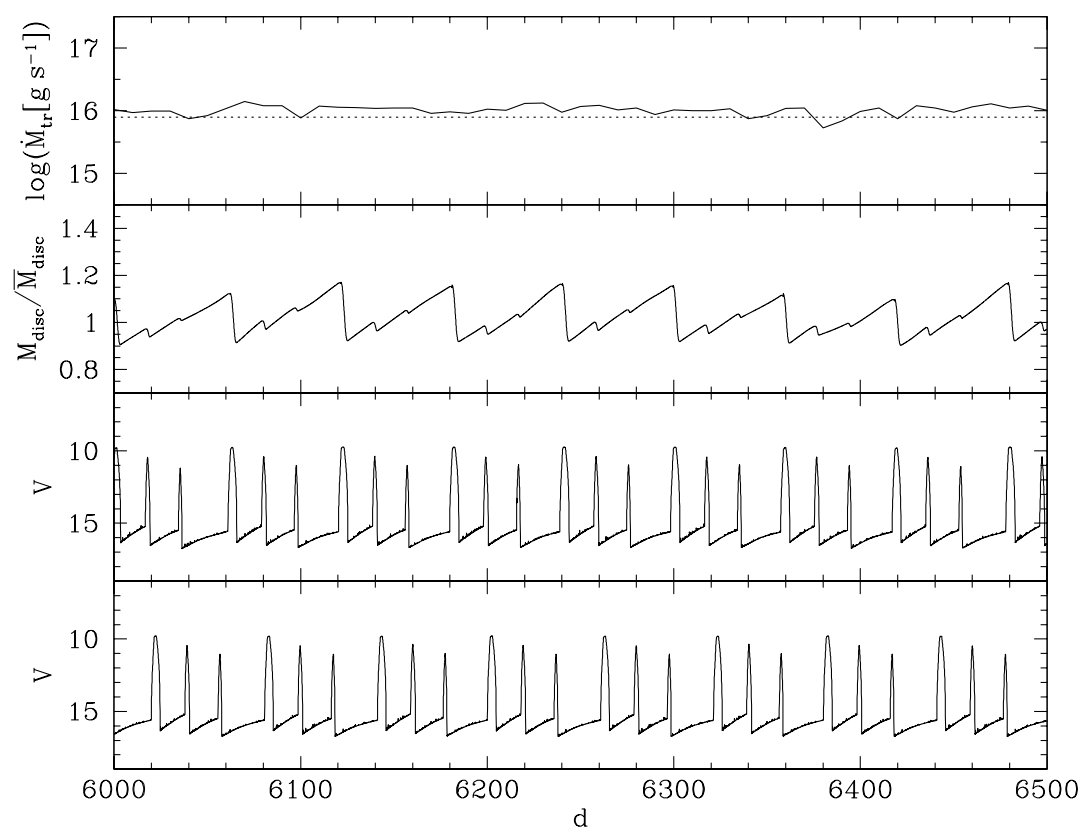

Figure 5.6: The same as Fig. 5.2 but a snapshot of the simulation where the transfer rate nearly stays constant.

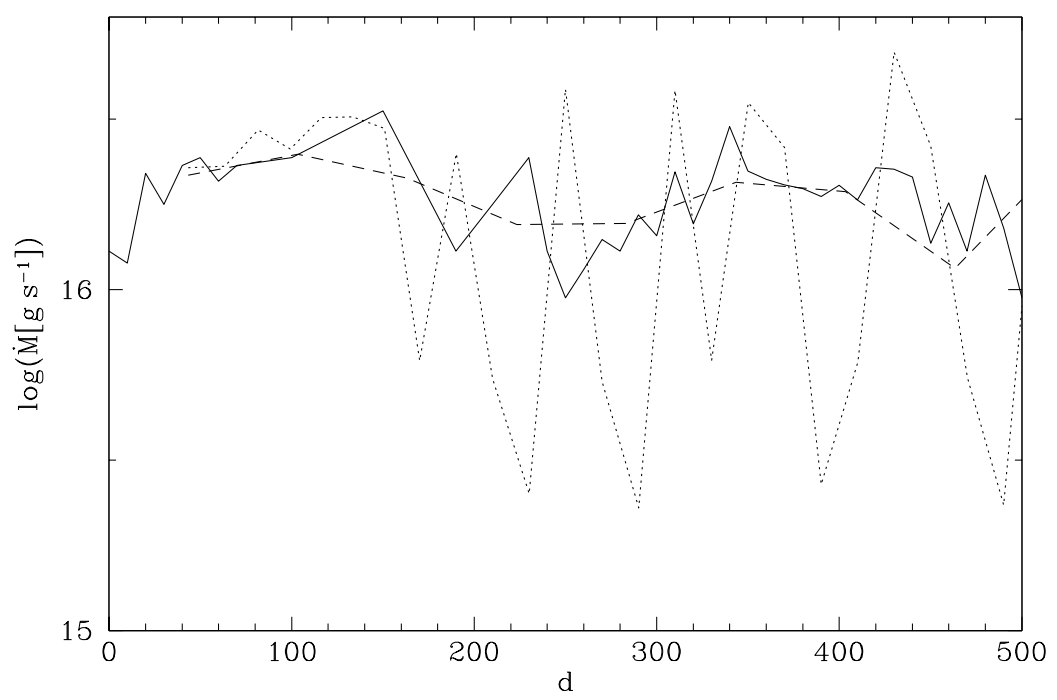

Figure 5.7: The mass transfer rate (solid line) and the accretion rate onto the white dwarf as function of time. The accretion rate is averaged over 60 days (dashed line) and averaged over 20 days (dotted line). 


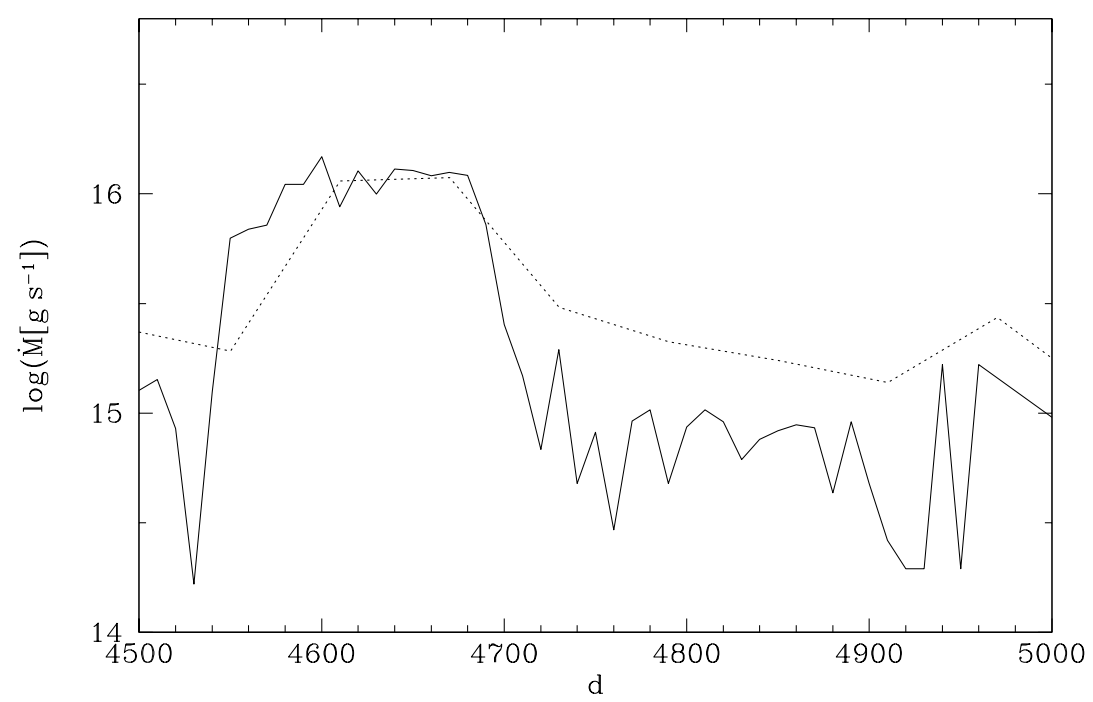

Figure 5.8: The mass transfer rate (solid line) and the accretion rate onto the white dwarf as function of time. The accretion rate is averaged over 60 days (dashed line).

mass estimates for AM Her, $M_{\mathrm{wd}}=0.39 M_{\odot}$ (Young et al. 1981), $M_{\mathrm{wd}}=0.69 M_{\odot}$ (Wu et al. 1995), $M_{\mathrm{wd}}=0.75 M_{\odot}$ (Mukai \& Charles 1987), $M_{\mathrm{wd}}=0.91 M_{\odot}$ (Mouchet 1993) and $M_{\mathrm{wd}}=1.22 M_{\odot}$ (Cropper et al. 1998). Based on the observed ultraviolet spectrum of AMHer and on its well-established distance, Gänsicke et al. (1998b) estimated $R_{\mathrm{wd}} \approx 1.1 \times 10^{9} \mathrm{~cm}$, and, using the Hamada \& Salpeter (1961) mass-radius relation for carbon cores, $0.35 M_{\odot}<M_{\mathrm{wd}}<0.53 M_{\odot}$. As the Hamada \& Salpeter mass-radius relation is valid for cold white dwarfs, the finite temperature $\approx 20000 \mathrm{~K}$ of the white dwarf in AM Her would allow also somewhat higher masses, $M_{\mathrm{wd}} \approx 0.65 M_{\odot}$, which is very close to the average mass of field white dwarfs, $0.6 M_{\odot}$, that I used.

Even though I exclude a massive white dwarf based on the observational evidences, I repeated my simulation with $M_{\mathrm{wd}}=1.0 M_{\odot}$ in order to test the dependence of my results on the white dwarf mass.

In a first step, I recomputed $\dot{M}_{\text {tr }}(t)$ from Eq. (5.3) with $M_{\text {wd }}=1.0 M_{\odot}$ and a corresponding $R_{\mathrm{wd}}=5.4 \times 10^{8} \mathrm{~cm}$. The resulting mean accretion rate is $3.0 \times$ $10^{15} \mathrm{~g} \mathrm{~s}^{-1}$, a factor 2.6 lower than before. Then, I simulated once more 7000 days of disc evolution with the new $\dot{M}_{\mathrm{tr}}(t), \alpha_{\mathrm{h}}=0.1, \alpha_{\mathrm{c}}=0.02$ and $R_{\text {out }}=2.8 \times$ $10^{10} \mathrm{~cm}$.

In Fig. 5.9 I show 500 days of my calculation with $M_{\mathrm{wd}}=1.0 M_{\odot}$. The disc produces only short outbursts and the outburst cycle of four outbursts with decreasing amplitude is hardly changed even by drastic variations of the mass transfer rate 


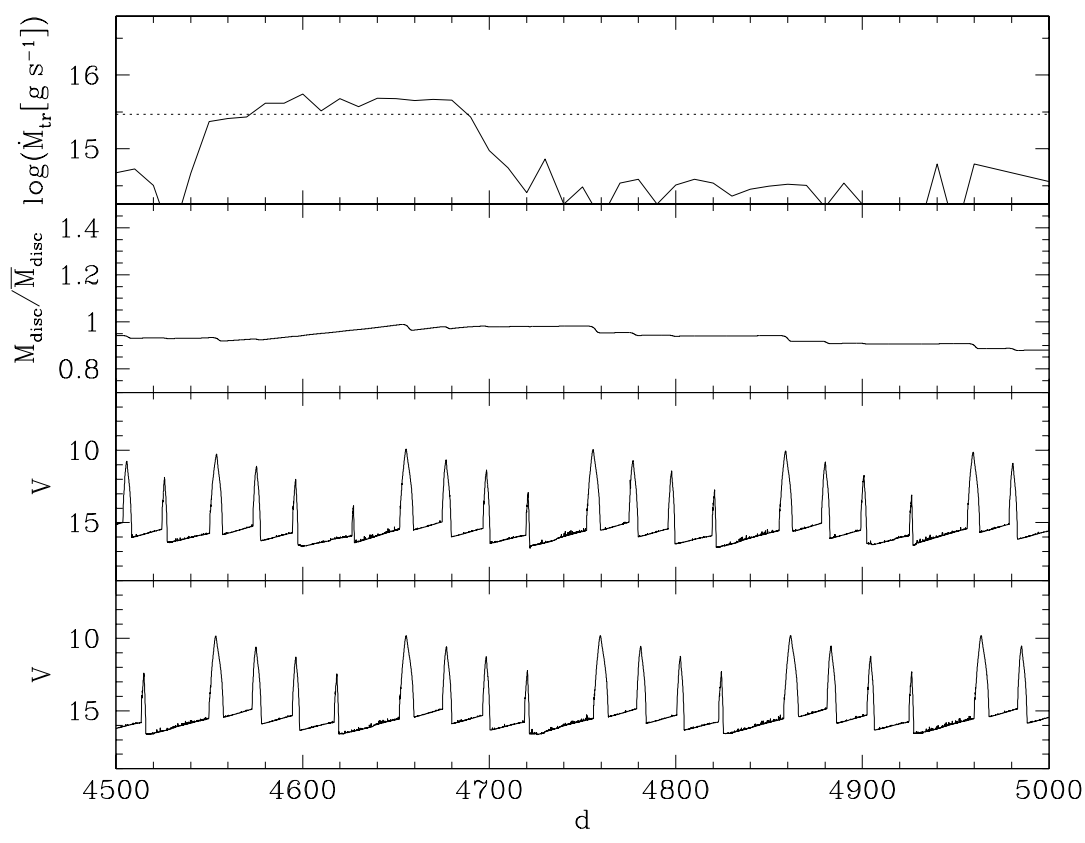

Figure 5.9: The same as in Fig. 5.4 but assuming a more massive primary $M_{\mathrm{wd}}=1.0 M_{\odot}$

(day 4700).

The different responses that my fictitious dwarf novae with $0.6 M_{\odot}$ and $1.0 M_{\odot}$ white dwarfs show to the variable mass transfer rate are easy to understand: both the increased primary mass and the decreased radius of the white dwarf reduce as mentioned above - the derived average mass transfer rate.

In addition, the outer disc radius of the fictitious system with $M_{\mathrm{wd}}=1.0 M_{\odot}$ increases. The disc becomes more massive, i.e. $\bar{M}_{\text {disc }}=4.59 \times 10^{23} \mathrm{~g}$, because of its increased size.

Due to the reduced mass transfer rate and the increased disc size, the heating waves are able to reach the outer edge of the disc only during the first outburst of the outburst cycle and only in cases where the mass transfer rate is high $\left(\log \dot{M}_{\mathrm{tr}} \geq\right.$ 16.1). Even in this rare situation the disc stays only a few days in the hot state and the white dwarf accretes only a small fraction of the disc mass $\left(\sim 1 / 8 M_{\text {disc }}\right)$. For lower transfer rates (the extremely more frequent case shown in Fig. 11) the heating front gets reflected before it has reached the outer edge of the disc. Hence, only a small percentage of the disc mass is involved in any outburst. Therefore, and due to the longer viscous timescale, the relaxation timescale given in Eq. (5.6) is always larger than a few years.

Summing up, the adopted primary mass and the average accretion rate play an 
important role on the influence that the variable mass transfer rate has on the outburst behaviour.

\subsection{Discussion and conclusions}

There is no reason to assume that the mass transfer variations of the secondary observed in AM Her are not present in non-magnetic systems. My numerical experiment including realistic variations of the mass transfer rate in a dwarf nova system is, therefore, a significant step towards a better understanding of dwarf nova light curves, and, thereby, of the underlying disc limit-cycle.

The light curve produced by my fictitious system switches between three states depending on the actual mass transfer rate. High transfer rates lead to only long outbursts where the entire disc is transformed into the hot state. If the transfer rate is near the average value the disc goes through a cycle of three outbursts, one long outburst followed by two short ones. Even long periods of low transfer rates do not force the disc to stop its outburst activity: long outbursts are suppressed and the duration of quiescence increases but the disc always produces short outbursts. From this follows that the low-states of VY Sculptoris stars (a subgroup of novalike variables) could not be caused by low transfer rates alone (see also Leach et al. 1999).

I find that in my fictitious system the mass accreted during an outburst cycle is dominated by the course of the mass transfer rate if the mass transfer rate varies significantly. The disc always relaxes to equilibrium with the mass input from the secondary. Thus, my experiment strongly supports King \& Cannizzo (1998) claim that dwarf nova accretion discs are probably never in a stationary state but are constantly adjusting to the prevailing value of $\dot{M}_{\text {tr }}$. Only during periods where the mass transfer rate is nearly exactly constant the disc periodically repeat the quasi stationary outburst cycle. Such periods are rare but occur in AM Her.

The strong influence of the mass transfer rate on the outburst behaviour of the fictitious system clearly indicates that probably most (if not all) the deviations from periodic outburst cycles seen in the light curves of dwarf novae are caused by variations of the mass transfer rate. 


\section{Chapter 6}

\section{Stream overflow and dwarf nova outbursts}

In the previous chapter I show that real variations of the mass transfer rate can strongly influence the outburst behaviour of the accretion disc. Another important boundary condition for the viscous evolution of accretion discs in CV's is the form of mass-input by the accretion stream from the secondary. Considering that the stream is geometrically thin - with typical height of order a few percent of the binary separation (Lubow \& Shu 1976) - the classic assumption has been that practically all the stream material is halted in its path by the outer disc. Lubow \& Shu (1976) showed, however, that the stream may be able to flow over and under the accretion disc. Smak (1985) suggested that this behaviour may be coupled with the local mass-accretion rate: during an eruption, the hot, relatively thick disc may stop the stream completely, whereas during quiescence the cold, relatively thin disc may permit stream overflow. Simple particle trajectory simulations by Hessman (1987) and Lubow (1989) and more elaborate fluid dynamical simulations by Livio et al. (1986) and Armitage \& Livio $(1996,1998)$ show how the overflowing material can skim relatively unimpeded over and under the disc until it finally collides with the inner disc. Hessman (1999) makes a detailed comparison of the stream and disc heights using the results of vertical disc structure calculations and shows that a large number of dwarf novae are likely to show overflow fractions of order $10 \%$ during quiescence. In addition, stream overflow is often referred to as the explanation for the observed dips in the light curves at orbital phases around 0.8 (Szkody et al. 1996; Long et al. 1996) in dwarf nova systems. In spite of that it was not yet taken into account in a satisfying manner in the thermal limit-cycle model.

In one of the earlier disc evolution calculations, Bath et al. (1983) directly included the effects of the stream by assuming that it is stripped by the disc at a rate which 
is proportional to the disc's surface density. Although the consequences of this model can even be derived analytically (Dgani \& Livio 1984), this admittedly arbitrary approach was not developed any further. Many investigators assume that all of the stream's mass, specific angular momentum, and energy is absorbed in some slightly extended region near the outer disc - either at a fixed radius (e.g. Cannizzo 1993a; Meyer \& Meyer-Hofmeister 1984; Mineshige 1988) or within a pre-defined radial extent which follows the outer disc radius (Ichikawa \& Osaki 1992). The former assumption does not permit the disc to change its radius freely in response to global redistributions of disc material (e.g. after large eruptions). Bath \& Pringle (1981) developed a mixing scheme for dynamically distributing the stream into the disc: if the local instantaneous mixture of all the disc and stream material possesses too little specific angular momentum, some fractions of the combined material is allowed to continue on to the next grid point. Though demonstrably better than simply dropping the material onto a spatially bounded region, the assumed instantaneity and the grid-dependence make this scheme less than ideal.

The light curves of dwarf novae have been used to constrain the properties of the effective viscosities in accretion discs - a topic of importance far beyond a mere interest in close binary systems. Thus, it is important to understand the possible role of stream penetration and overflow in these systems. In the next section, I review the equations for the viscous and thermal evolution of a disc, taking into account the effects of the stream. Thereafter, I present a simple but physically motivated model for the stripping of the stream by the disc. Finally, I show the effects on the outburst behaviour.

\subsection{The equations}

The classical equation describing the viscous evolution of the surface density $\Sigma$ in a geometrical thin, axisymmetric accretion disc is obtained by combining the vertically averaged Navier-Stokes and mass-conservation equations (see Eq. (3.38)). If I include the effects of external mass and specific angular momentum deposition, this equation assumes the form

$$
\begin{gathered}
\frac{\partial \Sigma}{\partial t}=\frac{3}{R} \frac{\partial}{\partial R}\left(R^{\frac{1}{2}} \frac{\partial}{\partial R}\left(R^{\frac{1}{2}} v \Sigma\right)\right)+\frac{1}{2 \pi R} \frac{\partial \dot{m}}{\partial R} \\
+\frac{1}{\pi R} \frac{\partial}{\partial R}\left(R\left(1-\frac{R_{J}^{\frac{1}{2}}}{R^{\frac{1}{2}}}\right) \frac{\partial \dot{m}}{\partial R}\right)
\end{gathered}
$$

(e.g. Bath et al. 1983), where again $v=(2 / 3) \alpha R_{\mathrm{g}} T / \mu \Omega$ is the kinematic viscosity. The first term on the right is the classical disc diffusion term, the second term 
describes the effects of external mass-deposition, and the third term is the result of angular-momentum deposition. $R_{J}$ is the circularisation radius where a Keplerian disc has the same specific angular momentum as the accretion stream. The masstransfer rate from the secondary must be equal to the integral of all the massdeposition rates in the disc:

$$
\dot{M}=\int_{R_{J}}^{R_{d}} \frac{\partial \dot{m}}{\partial R} d R
$$

The second equation one has to consider is the energy equation (in the central temperature $T_{\mathrm{c}}$ ) given in section 4.3 (Eq. (4.39)).

As the energy of the stream modifies the vertical structure and hence the loci of stable $\Sigma(\dot{M})$ in an unknown manner, I will ignore the contributions of the kinetic stream energy for now. Otherwise there would be an additional source term

$$
S_{e} \equiv \frac{1}{\pi R c_{p} \Sigma} \frac{\partial \dot{e}}{\partial R}
$$

where the local deposition of stream energy is given by

$$
\frac{\partial \dot{e}}{\partial R} \equiv \frac{\partial \dot{m}}{\partial R} \frac{1}{2}\left(v_{R}^{2}+\left(v_{\varphi}-v_{d}\right)^{2}\right) .
$$

Here $v_{R}$ and $v_{\phi}$ are the radial and azimuthal velocities of the stream and $v_{d}$ is the (azimuthal) velocity of the disc.

I solve the Eqs. (6.1) and (4.39) using the combined Finite-Element / FiniteDifference algorithm (FE for the spatial part and FD for the time-evolution) described in section 3.4. The upper ("outburst") and lower ("quiescent") branches of the "S-curves" are again characterised by constant quiescent and outburst values of $\alpha$. The thermal evolution is determined by some local cooling function $T_{\text {eff }}=T_{\text {eff }}(T, R, \Sigma, \alpha)$ derived from vertical structure calculations. For the purposes of this section, I adopt the same values of $\alpha\left(\alpha_{\mathrm{c}}=0.02\right.$ and $\left.\alpha_{\mathrm{h}}=0.1\right)$ and the simple power-laws fits to the cooling function given in Cannizzo (1993a). Finally, the optical light curves are calculated assuming that the disc is face-on, optically thick, and emits like a blackbody at the local effective temperature.

As mentioned above, Cannizzo (1993a) used orbital and disc parameters which are roughly appropriate for SS Cyg. There is no reason for believing that streamoverflow is an important process in this particular system, but most of the classical light curve analyses have been performed with the light curves of this system in mind. I therefore also adopt $d=100 \mathrm{pc}, M_{\mathrm{wd}}=1 \mathrm{M}_{\odot}, \dot{M}=10^{-9} \frac{\mathrm{M}_{\odot}}{\mathrm{yr}}, R_{\text {in }}=$ $5 \times 10^{8} \mathrm{~cm}$ and $R_{\text {out }}=4 \times 10^{10} \mathrm{~cm}$. 


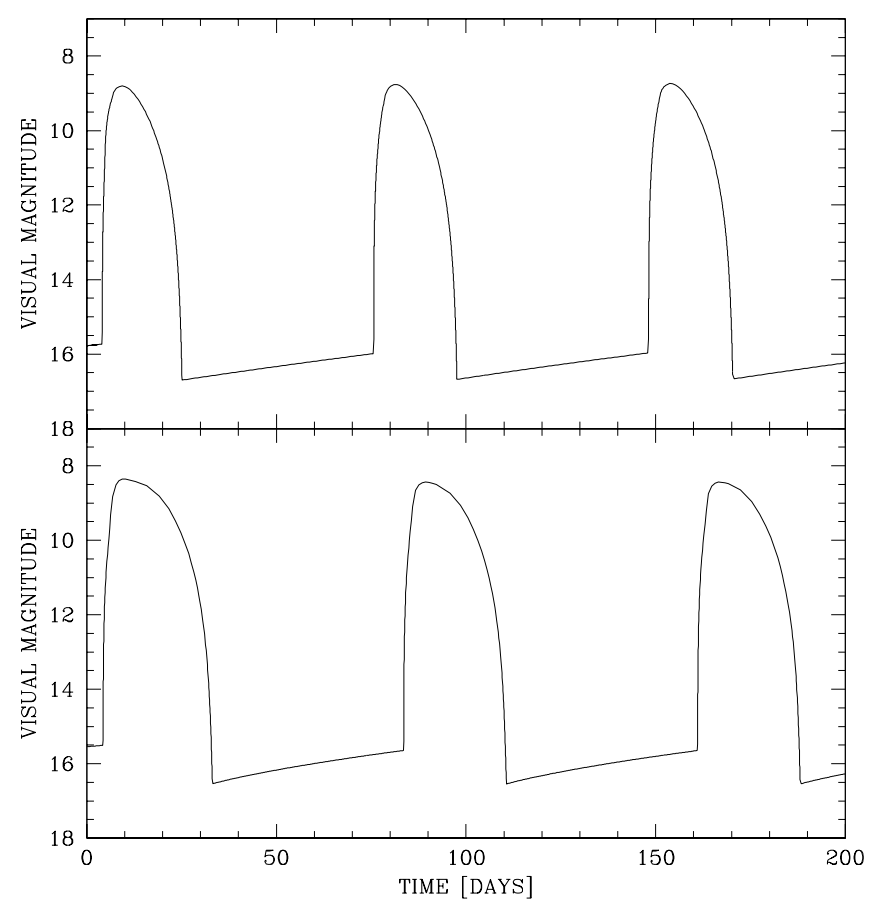

Figure 6.1: Optical light curves using (top) the stream deposition algorithm of Cannizzo (1993) and (below) using Bath \& Pringle's (1981) mixing scheme. The outbursts in the lower plot are a few days longer because the stream mass is added in a smaller region at the outer edge of the disc for the system parameters used.

\subsection{Stripping of the stream}

My model is motivated by the hydrodynamical simulations of Armitage \& Livio (1996). Assuming a stream which is geometrically thicker than but not as dense as the disc, they found that a substantial fraction of the original stream mass can "ricochet" off the disc edge and overflow towards smaller radii. This behaviour will occur when the disc is in quiescent state. If the disc is in the high state, the disc is thicker and less dense. The stream material then bores itself into the disc and is stopped at the outer edge. I therefore assume that the stream can be divided into two parts: an inner fraction which is always stopped at the outer disc; and the remaining outer fraction which can skim over and under the outer disc if these parts are cold, thin and dense.

The post-bright spot trajectory of the stream is described by the simple hydrodynamical solutions of Lubow \& Shu (1976) which assume that the stream has a 


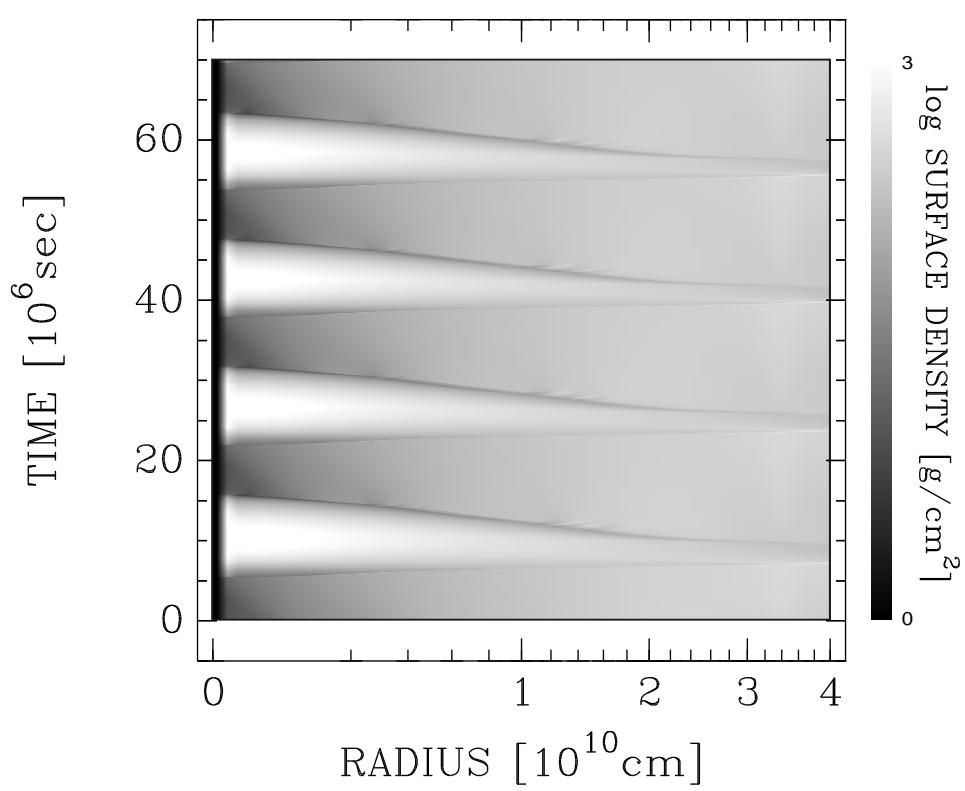

Figure 6.2: A gray-scale image showing the evolution of the surface density during four outbursts with radius and time using the same stream deposition assumptions as Cannizzo (1993a), starting with a disc in quiescent state. The accumulation of matter is represented by the increasing brightness mainly at the inner edge of the disc. The heating front from the inner disc which brings the whole disc into the high state and the cooling front which runs from the outer to the inner edge of the disc are clearly seen.

(radially varying) Gaussian cross-section so that

$$
\frac{\partial \dot{m}_{s}}{\partial z}=\frac{\dot{M}}{\sqrt{2 \pi} \sigma_{s}} \exp \left(-\frac{1}{2} \frac{z^{2}}{\sigma_{s}^{2}}\right) .
$$

I am mostly interested in systematic effects on the light curves of all dwarf novae rather than those for a particular system. These effects will mostly be dependent on the overflow mass-fraction $f \equiv \frac{\dot{M}_{o v}}{\dot{M}}$, so I simply use $f$ as a model parameter for the purposes of this initial study and so arbitrarily selecting the stream properties $\sigma_{s}(f)$ which result in a given mass-overflow fraction $f$ in the quiescent state directly after an eruption.

The disc height is approximated by the pressure scale height:

$$
H=\sqrt{\frac{R_{g} T}{\Omega^{2}}} .
$$

For a given effective outer disc height $H\left(R_{\text {out }}\right)$, the corresponding stream height at that radius - and hence the vertical distribution of stream mass-density - is a simple function of the assumed quiescent overflow mass-fraction $f$. 
The radial distribution of the deposition of stream mass and angular momentum (the terms with $\frac{\partial m}{\partial R}$ in Eq. (6.1)) is then determined by the instantaneous relative heights of the disc and the underside of the stripped stream. The stream material is distributed by comparing this heights at every spatial node $\left(R_{i}\right)$ of the FE-grid, starting at the outer disc radius and working inwards until either the stream is effectively "used up" or the circularisation radius is reached. In the latter case, the remaining stream material is simply deposited at that grid-point. At nodes where the disc height exceeds the underside of the stream the local deposition rate is

$$
\dot{M}_{i}=2 \int_{H_{s}}^{H_{d}} \frac{\partial \dot{m}_{s}}{\partial z} d z
$$

Here $H_{s}$ and $H$ are the instantaneous heights of the underside of the stream and the disk height at $R_{i} . H_{s}$ is, of course, zero beyond the outer edge of the disc. For numerical reasons these local deposition rates are smeared out using a Gaussian distribution with a width of a few grid-points. This procedure leads to the following form for the mass-deposition rate:

$$
\frac{\partial \dot{m}}{\partial R}=\sum_{i=1}^{n} \frac{\dot{M}_{i}}{\sqrt{2 \pi} \sigma_{\text {smear }}} \exp \left(-\frac{1}{2} \frac{\left(R-R_{i}\right)^{2}}{\sigma_{\text {smear }}^{2}}\right) .
$$

where $\dot{M}_{i}$ is given by Eq. (6.7).

\subsection{Results}

I first recalculated the dwarf nova light curves discussed in detail in Cannizzo (1993a) using the same stream deposition assumptions, both to check the reliability of my code and as a means of comparing the disc evolution with and without the effects of stream overflow. In addition, I calculated the light curves using Bath \& Pringle (1981) mixing scheme. The results can be seen in Fig. 6.1: for the parameters of SS Cygni, either assumption produces essentially the same light curve. This is not surprising, since large variations in the disc radius are mainly expected for short period systems (the SU UMa subclass of the dwarf novae: Ichikawa \& Osaki (1992).

The evolution of the disc surface density $\Sigma$ for the case of no stream overflow $(f=0)$ using Cannizzo's method of mass addition is shown as a gray-scale plot in Fig. 6.2. One sees the qualitative characteristics of a dwarf nova eruption triggered by a disc instability. During quiescence, the surface density increases with radius. As the surface density rises, some annulus in the disc finally goes into the hot, outburst state and initiates a "heating-front" which sends the whole disc into the 


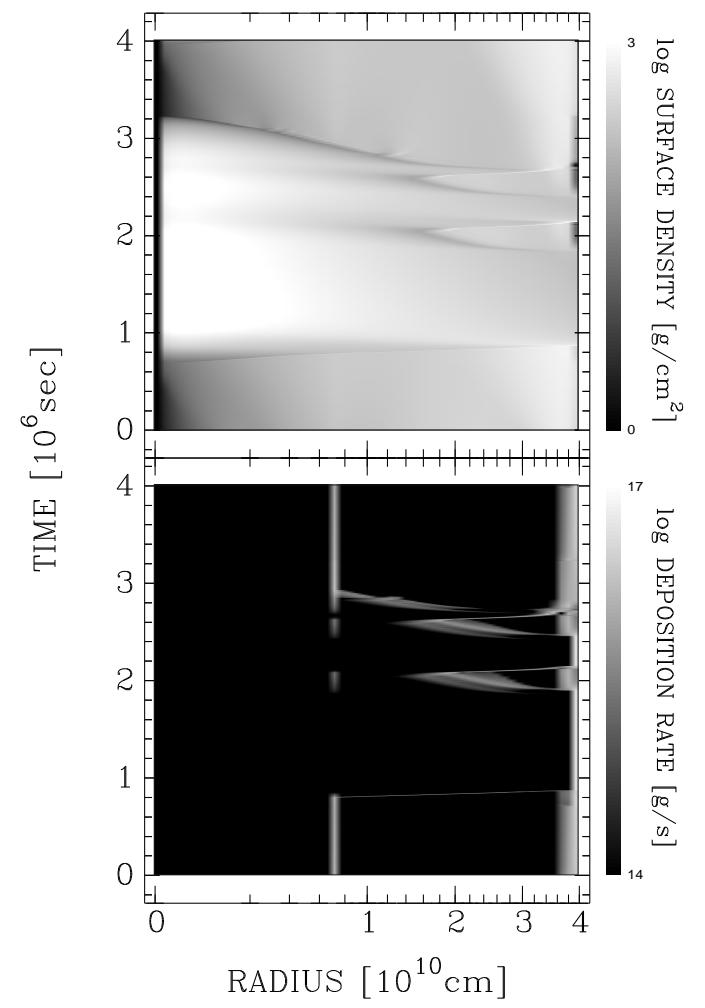

Figure 6.3: Evolution of the (top) surface density and (bottom) massdeposition rate during one outburst for the case of $30 \%$ stream overflow, showing how the stream interacts with the heating and cooling fronts (compare with Fig. 6.2).

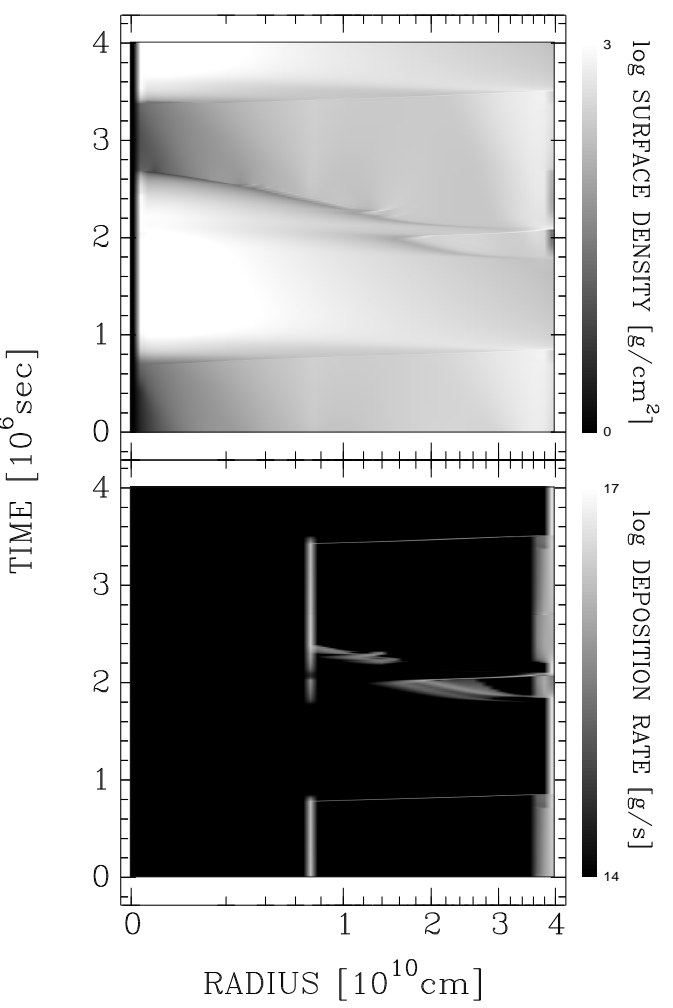

Figure 6.4: Same as Fig. 6.3, but for a mass-overflow fraction $f=0.4$.

hot state. For the disc parameters which I used, this occurs in the inner disc. At the height of the eruption, the disc is nearly in steady-state, with a surface density which now decreases with radius. The rate of accretion onto the central object now exceeds the rate at which matter is being added to the disc from the mass losing star. Eventually, the surface density drops down to the critical density for a transition back to the quiescent state in the outer disc, and a "cooling-front" emerges, bringing the disc back to quiescence.

In order to test the effects of more realistic stream stripping, I calculated the evolution of the disc using (arbitrary) stream parameters corresponding to overflow fractions $f$ equal to $0.1,0.2,0.3$, and 0.4 . These values are determined using the heights from a disc which is in a quiescent state immediately after outburst (there- 
fore these values represent maximal stream overflow rates for the chosen parameters). For the system parameters of SS Cyg and my simple model for stream overflow, the outburst behaviour is not essentially changed unless the amount of stream overflow is greater then $25 \%$ of the mass transfer rate.

The standard picture shown in Fig. 6.2 changes as the amount of stream overflow exceeds $25 \%$. The evolution of the surface density and deposition rate in the case of $f=0.3$ is shown in Fig. 6.3. When the disc is in the quiescent state, the effect of stream overflow is not obvious because the deposition of mass in the inner disc does not change the overall accumulation of matter. The critical value of the surface density is again reached at the inner edge of the disc. While the heating front is running through the disc, the radius at which the overflowing stream mass is stopped by the disc travels outward (best seen in the bottom part of Fig. 6.3). This is not surprising, because the hot inner regions of the disc are geometrically thicker. Because mass is added at the position of the heating front directly from the stream, the heating front is faster than in the non-overflow case. When the entire disc is in the high-state, all of the stream mass is added at the outer edge and the behaviour of the disc is the same as in the classical picture. The influence of stream overflow again becomes important when the cooling front starts to turn the disc back into its low state. The addition of mass directly into the cooling front causes a new heating front before the cooling front has reached the inner edge of the disc. This heating front returns the disc to the nearly steady-state high state. This procedure recurs until there is not enough mass in the outer disc to sustain a high state and the disc finally returns to true quiescence.

If the fraction of overflowing stream mass is $40 \%(f=0.4)$, the outburst behaviour and evolution of the surface density (Fig. 6.4, top) is similar to that shown in Fig. 6.3 but with an important difference: when the cooling wave has started, a significant fraction of the overflowing stream material is not added directly into the cooling front because the stream thickness is large enough to let stream mass overflow both the cold and hot parts of the outer disc (Fig. 4, bottom). Therefore, there is not enough mass stored in the outer disc to maintain the secondary high state and the third heating front does not start.

The light curves for $f=0.0,0.2,0.3$, and 0.4 are shown in Fig. 6.5. In the top panel I used the same stream deposition assumptions as Cannizzo (1993a), i.e. $f=0$. If the amount of stream overflow is below 25\% (second panel), the light curves are similar to the light curves computed with Bath \& Pringle (1981) mixing scheme. The similarity to Bath and Pringle's light curves is caused by the small Gaussian distributions which I used for smearing out the local deposition rates $\left(\sigma_{\text {smear }}\right.$ in Eq. (6.5)). The outbursts are a few days longer because the mass addition mainly occurs in a smaller region. The amount of stream overflow is not yet sufficient to systematically change the form of the light curve. As $f$ 


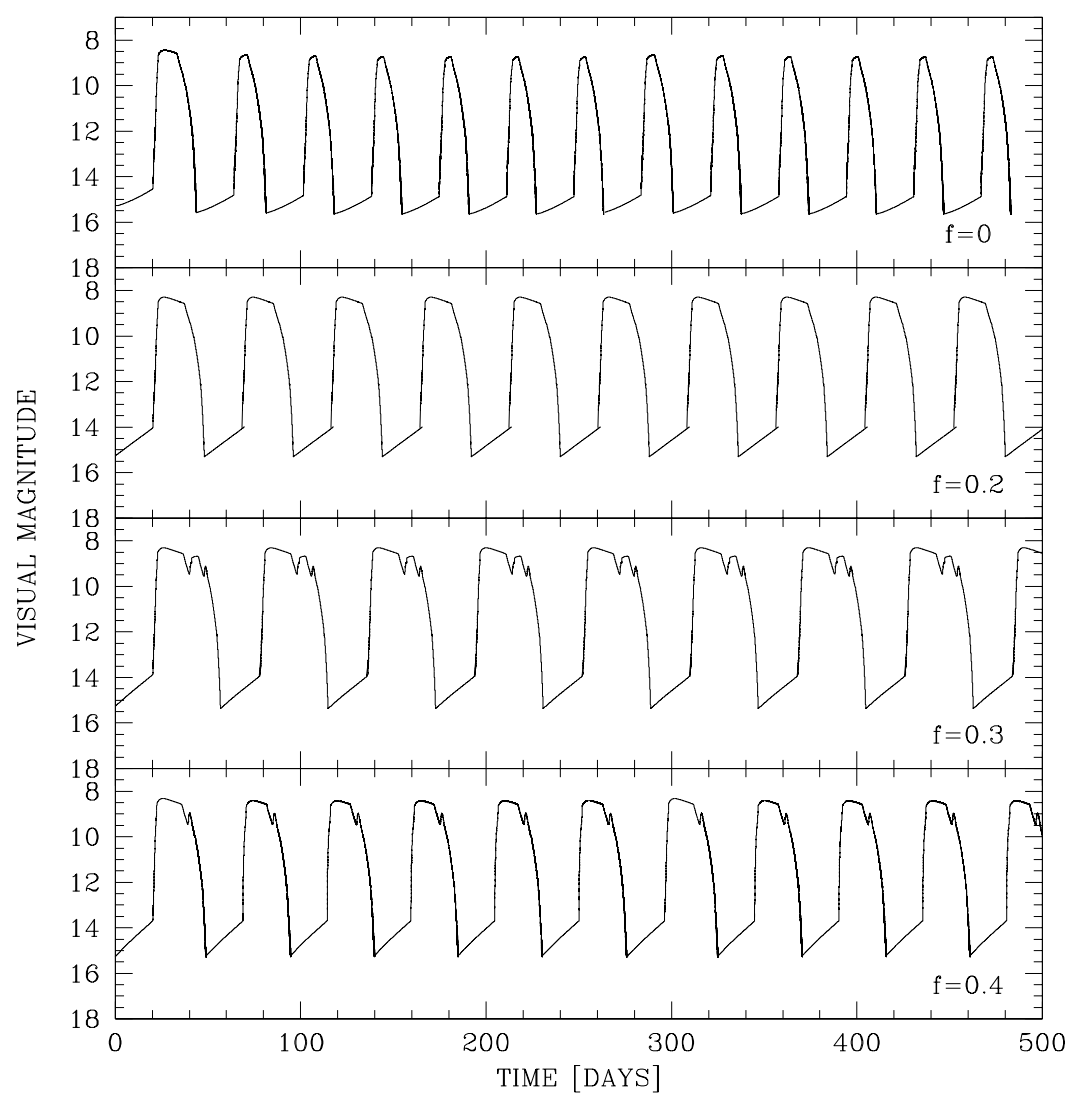

Figure 6.5: Light curves computed for different values of the mass-overflow fraction $f$ (see text).

is increased further ( 0.3 in the lower middle panel), the light curve develops two peaks in the declining phase because of the early re-heating of the cooling disc by the stream. As discussed above, this effect is smaller in the case of $f=0.4$ because there is less mass added directly from the stream into the cooling front. Nevertheless, the length of the outburst has been considerably increased over the case $f=0$.

\subsection{Conclusions}

I have calculated light curves using the classical thermal limit-cycle model for dwarf nova eruptions and several different methods for the addition of accretion stream mass and angular momentum to the disc. In contrast to previous studies, I have included the effects of realistic stream overflow, parameterised as the 
overflow mass-fraction $f$. I calculated the amount of matter and angular momentum deposited by the overflowing stream by comparing the pressure scale height of the disc and the trajectory of the local underside of the assumed undisturbed stream. While this is a rough approximation, it represents the basic physical situation and is supported by the results of numerical hydrodynamical simulations of the stream-disc collision.

When significant stream overflow occurs, the stream not only changes the pattern of matter-deposition in the quiescent disc, but interacts with any heating and cooling fronts. When the amount of stream overflow exceeds $25 \%$, the stream can reverse an inward travelling cooling front and create an outward-travelling heating front. The resulting light curves show two or more peaks during the declining phase. This effect nearly disappears for overflow fractions greater than $40 \%$ because the amount of mass added directly from the stream into the cooling front becomes smaller.

This study suggests that only very large overflow fractions can change the outbursts of dwarf novae. I have chosen the system parameters of SS Cyg in this study, only because of the central role the light curves of this system have played in the development of the thermal limit-cycle model and of $\alpha$-model discs, therefore it is not yet clear if this conclusion is more generally true. As mentioned above, Hessman (1999) found overflow fractions of $\lesssim 10 \%$ by comparing the stream and disc heights for many dwarf nova, which further indicates that stream overflow only marginally affects the outburst light curves. 


\section{Chapter 7}

\section{Irradiated accretion discs around white dwarfs}

Irradiation is a very important phenomenon in most types of accretion discs. Reprocessed disc irradiation is thought to contribute significantly to the emission in Super Soft Sources (e.g. Matsumoto \& Fukue 1998), X-Ray Binaries (e.g. Dubus et al. 1999; King 1998) and AGN (e.g. Guilbert \& Rees 1988; Burderi et al. 1998). External irradiation of the disc by the hot central source can suppress the thermal instabilities especially in the inner disc regions. In the context of soft X-ray transients, Tuchman et al. (1990) and Mineshige et al. (1990) calculated the thermal equilibrium structure of externally irradiated accretion disc annuli assuming that the irradiation flux is thermalised in the photosphere of the disc. King (1997) explained the UV-delay in dwarf novae taking into account irradiation from the white dwarf which truncates the inner disc. Hameury et al. (1999) confirmed this result only for hot white dwarfs $\left(T_{\mathrm{wd}} \geq 40000 \mathrm{~K}\right)$ and found that the depletion of the inner disc must create several small outbursts between the main outbursts, contrary to observations. Leach et al. (1999) explained the low states of VY Scl stars as being due to the interplay of irradiation from hot white dwarfs and low mass transfer rates but did not consider the influence which irradiation has on the disc structure and, thereby, on the S-curve.

For completeness I note that the motivation of King (1997) and Hameury et al. (1999) was to account for the "problem of the UV delay" in dwarf novae although the effects of irradiation of the inner disc are of general importance for the operation of the accretion disc limit-cycle mechanism. The "problem of the UV delay" results from observations of several dwarf nova (e.g. VW Hydri) which show that the rise in the ultraviolet flux lags behind that in the optical by as much as $12 \mathrm{hr}$ or more (see Livio \& Pringle 1992, for a list of dwarf novae with observed UV delay). Smak (1998) critically re-examined the basis for the claim of a problem, 
and found that there does not seem to be one. Previous studies which had claimed there to be a problem had utilised various simplifications in their time-dependent models which turned out to be critical - such as the neglect of a variable outer boundary. The contraction of the accretion disc during quiescence has the effect of increasing the local surface density in the outer disc, and, thereby, promoting disc instabilities which begin at large radii. These so-called "outside-in" (Cannizzo et al. 1986) or "type A" (Smak 1984) outbursts in which a narrow spike of enhanced surface density propagates from the initial site of the instability to the inner edge, are able to produce outbursts with the observed delay.

If disc irradiation accounts for the UV delay or not, disc accretion onto white dwarfs provides a supreme possibility to study the dynamical behaviour of irradiated accretion discs as the irradiation geometry of the system is rather simple and the dynamical behaviour can be examined by analysing the observed light curves. As the white dwarf in post novae systems is extremely hot compared to the white dwarfs in "normal" CVs (section 2.3) it is specifically promising to study the influence of disc irradiation by the hot white dwarf in these systems.

In this section I include irradiation from the white dwarf in the calculations of the accretion disc structure and discuss the impact on the outburst light curves. At first I present calculations of the vertical structure considering the effects of external irradiation. Thereafter I derive approximations for the cooling functions from these detailed calculations and include them into the theoretical description of disc outbursts. Finally, I discuss the effects of disc irradiation on the dynamical behaviour of the accretion discs in the context of dwarf novae and post novae.

\subsection{Vertical structure}

In section 4.2 I presented the standard equations describing the vertical disc structure. The outer boundary was defined by:

$$
T^{4}\left(\tau=\frac{2}{3}\right) \equiv T_{\mathrm{ph}}^{4}=T_{\mathrm{eff}}^{4},
$$

where $T_{\mathrm{ph}}$ denotes the photospheric or surface temperature. In case the disc annulus is irradiated by an external source (e.g. the white dwarf) the boundary condition above is changed in order to take into account the irradiating flux:

$$
T_{\mathrm{ph}}^{4}=T_{\mathrm{vis}}^{4}+T_{\mathrm{irr}}^{4} .
$$

where I replaced $T_{\text {eff }}$ with $T_{\text {vis }}$ as the term $\sigma T_{\text {vis }}^{4}$ now represents only the contribution of viscous heating to the photospheric temperature. The irradiation tempera- 
ture $T_{\text {irr }}$ is related to the irradiating flux by:

$$
\sigma T_{\mathrm{irr}}^{4}=F_{\mathrm{irr}}=\int_{0}^{\infty} F_{\lambda}^{\mathrm{irr}} d \lambda
$$

As the approximation given in Eq. (7.2) does not consider the structure of the disc above the photosphere, it is not clear how efficient a given amount of irradiation can be in heating up the disc photosphere. In addition, by using Eq (7.2) only the integrated irradiation flux is considered, the actual spectral energy distribution of the illumination is neglected. These uncertainties are parameterised in the disc albedo $\beta$ which might be different for different spectral energy distributions of $F_{\text {irr. }}$

I computed the vertical structure of an irradiated disc annulus using the approximation given in Eq. (7.2) and the earlier presented equations (4.16)-(4.21). Fig. 7.1 presents examples of resulting vertical structures in which the influence of irradiation is shown. One can clearly see that irradiation suppresses convection and flattens the temperature profile, but even when the irradiation flux strongly exceeds the viscous heating of the photosphere $\left(F_{\text {irr }} \sim 15 F_{\text {vis }}\right.$ for $T_{\text {irr }}=12000 \mathrm{~K}$ in Fig. 7.1) the disc is not isothermal.

As I described in chapter 4, the radial structure can be separated from the vertical structure calculations, but it is necessary to obtain cooling functions from the vertical structure results. In order to derive these cooling functions I calculated a grid of vertical accretion disc structures. For every set of $\alpha, M_{\mathrm{wd}}$ and $R$ I calculated $S$ curves for irradiation temperatures $T_{\text {irr }}$ between 0 and 12000 K. Figs. 7.2 and 7.3 show examples of thermal equilibrium curves including external irradiation for the accretion rate, the photospheric, viscous and central temperature as a function of $\Sigma$. One can clearly see that irradiation suppresses the occurrence of the S-shape and, hence prevents disc outbursts. This results from the supression of convection even for low accretion rates.

Using the resulting database I derive expressions for the critical accretion rates and the critical surface densities using simlpe power law fits. Throughout this thesis the index "+" refers to critical values of the upper, hot branch of the S-curve (below which the hydrogen in the disc starts to recombine and the disc becomes unstable) whereas the index "-" denotes critical values of the lower, cold branch (above which the hydrogen becomes partially ionised). For the non-irradiated disc (i.e. $T_{\text {irr }}=0$ ), I obtain

$$
\begin{aligned}
& \dot{M}_{+}=9.5 \times 10^{15} \mathrm{~g} \mathrm{~s}^{-1} R_{10}^{2.64} \alpha_{\mathrm{h}}^{0.01} M_{\mathrm{wd}}^{-0.88}, \\
& \dot{M}_{-}=4.0 \times 10^{15} \mathrm{~g} \mathrm{~s}^{-1} R_{10}^{2.66} \alpha_{\mathrm{c}}^{-0.001} M_{\mathrm{wd}}^{-0.88},
\end{aligned}
$$

and

$$
\Sigma_{+}=8.0 \mathrm{~g} \mathrm{~cm}^{-2} R_{10}^{1.11} \alpha_{\mathrm{h}}^{-0.75} M_{\mathrm{wd}}^{-0.38}
$$




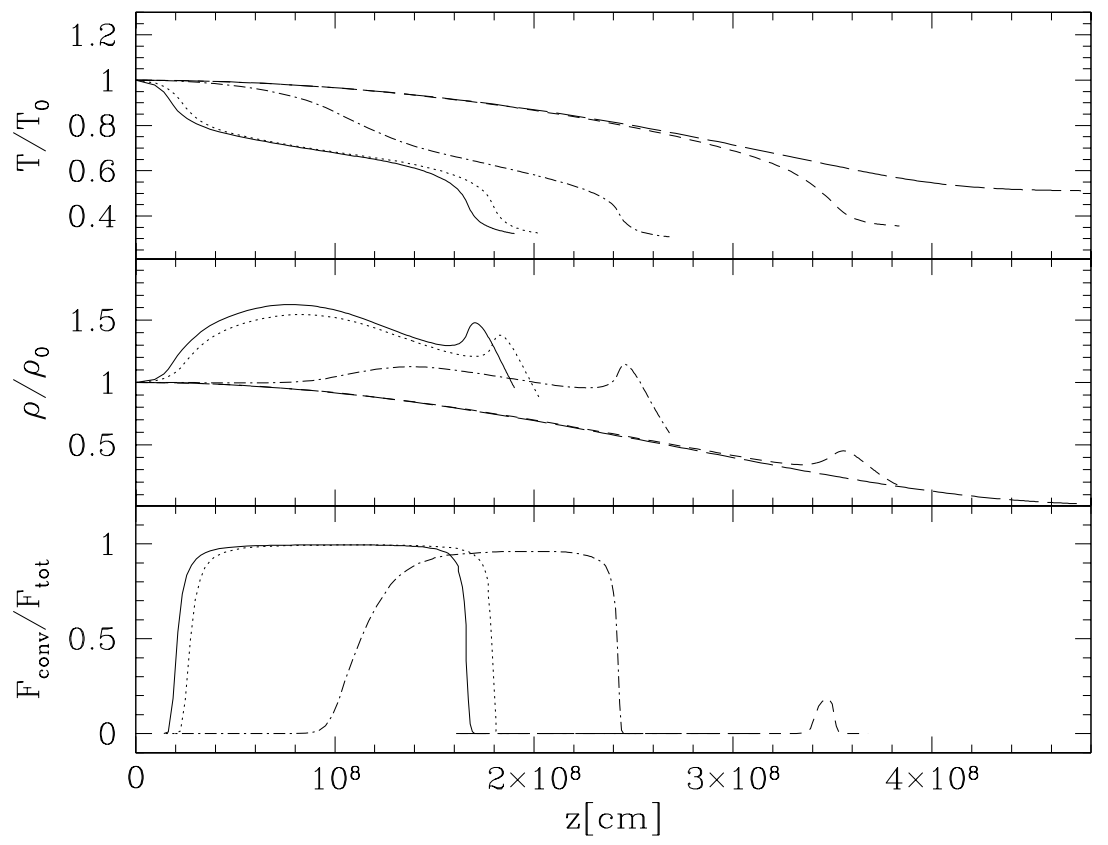

Figure 7.1: The temperature, the density and the contribution of convection to the energy transport as functions of $\mathrm{z}$ for an annulus at $R=1.3 \times 10^{10} \mathrm{~cm}, \alpha=0.2 \dot{M}_{\text {acc }}=$ $1.5 \times 10^{-10} M_{\odot} \mathrm{yr}^{-} 1, M_{\mathrm{wd}}=1.1 M_{\odot}$ and different amounts of irradiation. I used $T_{\text {irr }}=0$ (solid lines), 4 (dotted line), 6 (dashed-dotted lines), 8 (short dashed lines), 12 (long dashed line) $\times 10^{3} \mathrm{~K}$. External irradiation suppresses convection because it flattens the temperature profile of the disc. For $T_{\text {irr }}=12000 \mathrm{~K}$ the energy transport through the disc is radiative although it was fully convective without irradiation. The disc height and the central temperature increase with increasing irradiation $\left(T_{0}=18450 \mathrm{~K}\right.$ for $T_{\text {irr }}=0$ and $T_{0}=23823$ for $T_{\text {irr }}=12000 \mathrm{~K}$ ). The values of the central density are ranging from $\rho_{0}=9.2 \times 10^{-8} \mathrm{~g} \mathrm{~cm}^{-3}$ without irradiation to $\rho_{0}=3.9 \times 10^{-8} \mathrm{~g} \mathrm{~cm}^{-3}$ for $T_{\text {irr }}=12000 \mathrm{~K}$.

$$
\Sigma_{-}=12.8 \mathrm{~g} \mathrm{~cm}^{-2} R_{10}^{1.14} \alpha_{\mathrm{c}}^{-0.78} M_{\mathrm{wd}}^{-0.38},
$$

where $R_{10}=R / 10^{10}[\mathrm{~cm}], M_{\mathrm{wd}}$ the white dwarf mass in solar units and $\alpha_{\mathrm{c}}$ and $\alpha_{\mathrm{h}}$ the viscosity parameter for the cold and hot branch, respectively. Eqs. (7.4)(7.7) are in agreement with earlier derived expressions (e.g. Hameury et al. 1999; Cannizzo 1993a; Ludwig et al. 1994).

As noted above, irradiation has a significant influence on the ionisation state of the disc. The critical accretion rate, below which the disc becomes unstable, decreases with increasing irradiation until the external flux becomes strong enough to hold the disc in the hot and stable state independent of the accretion rate. I find that the irradiation temperature $T_{\text {irr,s }}$ for which $\dot{M}_{\text {cr }}$ vanishes is a slightly decreasing 

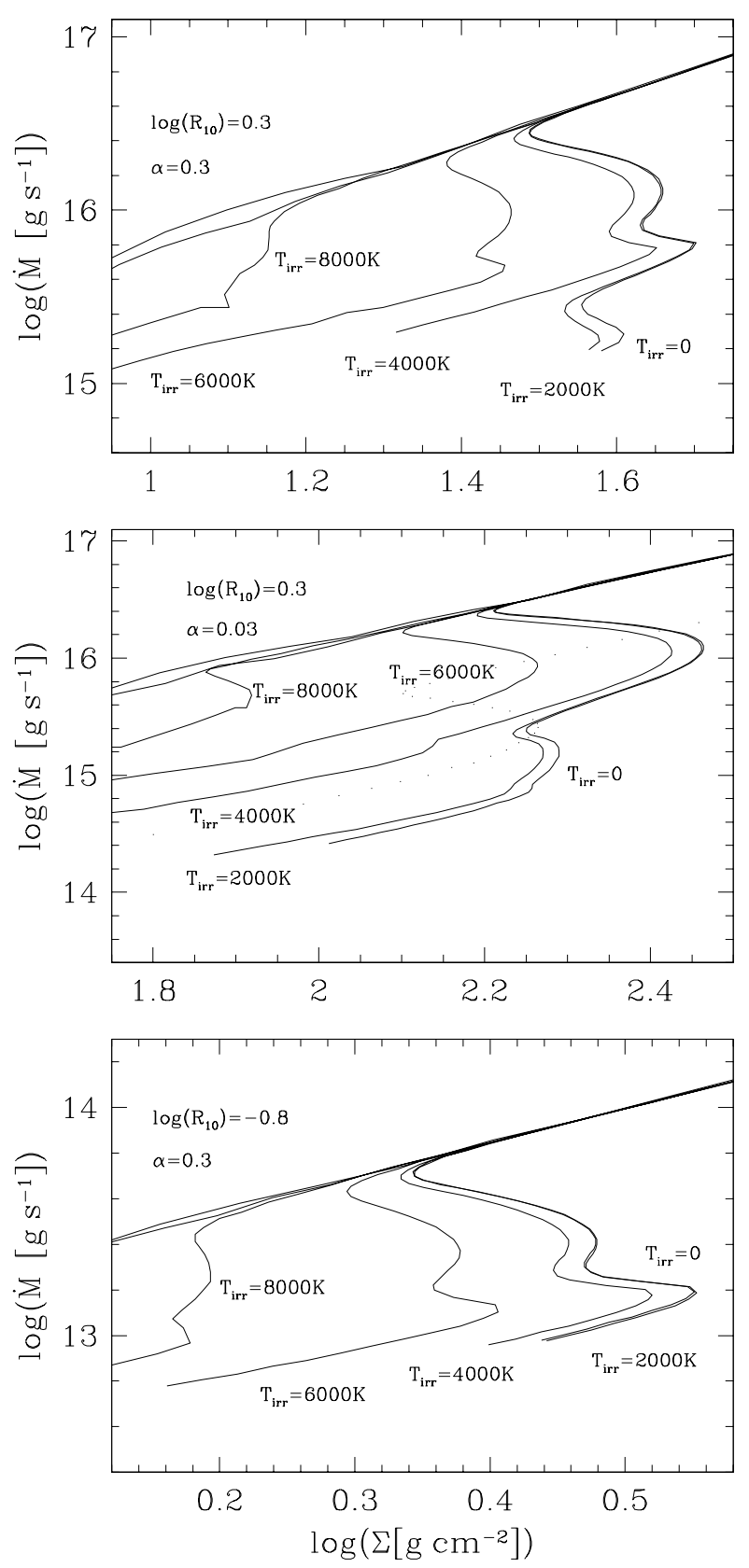

Figure 7.2: The vertical equilibrium curves for irradiation temperatures of $T_{\text {irr }}=$ $[0,2,4,6,8,10,12] \times 10^{3} \mathrm{~K}$ (right to left) and a white dwarf mass of $M_{\mathrm{wd}}=1.3 M_{\odot}$. Assuming a relatively high $\alpha$ and a large radius, the disc instability is suppressed for an irradiation temperature $T_{\text {irr }}=8000 \mathrm{~K}$ (top panel), while it is not fully suppressed for a lower $\alpha$ (middle panel) or at a smaller radius (bottom panel) and the same irradiation temperature $T_{\text {irr }}=8000 \mathrm{~K}$. The external irradiation temperature, necessary to suppress the instability, increases with decreasing radius and decreasing $\alpha$ (see Eq. (8)). Note the different scalings. 

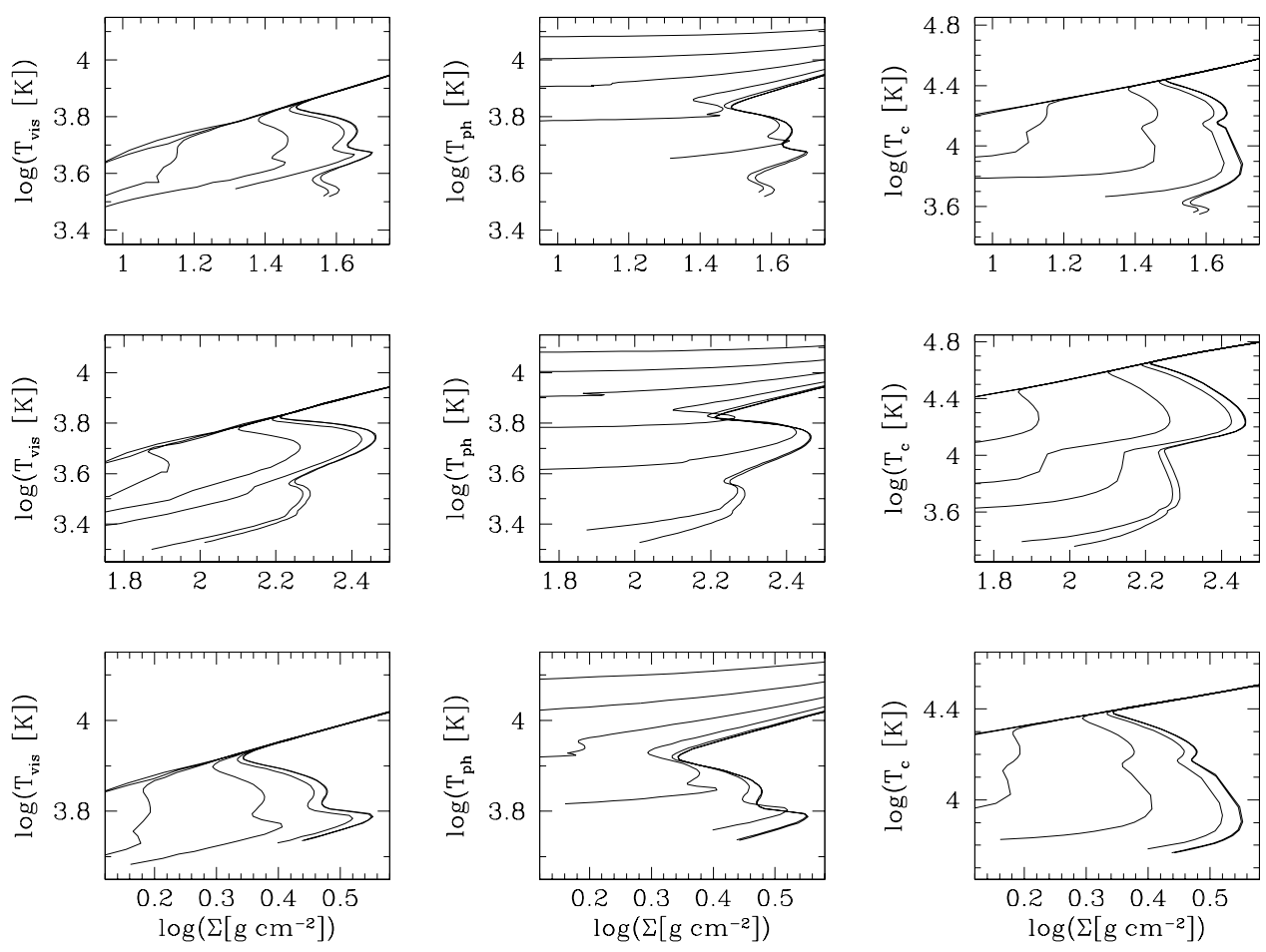

Figure 7.3: The vertical equilibrium curves for irradiation temperatures of $T_{\text {irr }}=$ $[0,2,4,6,8,10,12] \times 10^{3} \mathrm{~K}$ (right to left), a white dwarf mass of $M_{\mathrm{wd}}=1.3 M_{\odot}$. The top panels show calculations using $\alpha=0.3, R_{10}=0.5$. In the panels below I use a smaller $\alpha$ (i.e. $\alpha=0.03$ ) and in the bottom panels I use again $\alpha=0.3$ but $R_{10}=1.6$. The corresponding $\dot{M}$ curves can be found in Fig. 7.2.

function of $R_{10}$ and $\alpha$, but nearly independent of $M_{\mathrm{wd}}$ :

$$
T_{\text {irr }, \mathrm{s}}=7382 \mathrm{~K} \alpha^{-0.07} R_{10}^{-0.03}
$$

The decrease of the critical accretion rates and surface densities between the nonirradiated case and the suppression of the instability is approximately given by:

$$
\begin{aligned}
& \dot{M}_{+}^{\mathrm{irr}}=\dot{M}_{\mathrm{cr}}\left(1-\frac{T_{\mathrm{irr}}^{7.2}}{T_{\mathrm{irr}, \mathrm{s}}^{7.2}}\right), \\
& \dot{M}_{-}^{\mathrm{irr}}=\dot{M}_{\mathrm{cr}}\left(1-\frac{T_{\mathrm{irr}}^{4.5}}{T_{\mathrm{irr}, \mathrm{s}}^{4.5}}\right),
\end{aligned}
$$


and

$$
\begin{aligned}
& \Sigma_{+}^{\mathrm{irr}}=\Sigma_{+}\left(1-\frac{T_{\mathrm{irr}}^{3.7}}{T_{\mathrm{irr}, \mathrm{s}}^{3.7}}\right), \\
& \Sigma_{-}^{\mathrm{irr}}=\Sigma_{-}\left(1-\frac{T_{\mathrm{irr}}^{6.3}}{T_{\mathrm{irr}, \mathrm{s}}^{6.3}}\right) .
\end{aligned}
$$

For the central temperature (Fig. 7.3, right panels) I find in the absence of irradiation:

$$
\begin{aligned}
& T_{\mathrm{c},+}=35500 \mathrm{~K}\left(\frac{\alpha_{\mathrm{h}}}{0.1}\right)^{-0.2} M_{\mathrm{wd}}^{-0.014} R_{10}^{0.043} \\
& T_{\mathrm{c},-}=12620 \mathrm{~K}\left(\frac{\alpha_{c}}{0.05}\right)^{-0.2} M_{\mathrm{wd}}^{-0.01} R_{10}^{0.03}
\end{aligned}
$$

in good agreement with Ludwig \& Meyer (1998). I find $T_{\mathrm{c},-}$ somewhat increasing but - especially in the case of low $\alpha$ - nearly independent of irradiation whereas $T_{\mathrm{c},+}$ decreases with increasing irradiation. In the calculations which I present in this section I use the following approximations:

$$
\begin{aligned}
& T_{\mathrm{c},+}^{\mathrm{irr}}=T_{\mathrm{c},+}\left(1-\frac{T_{\mathrm{irr}}^{2.5}}{T_{\mathrm{irr}, \mathrm{s}}^{2.5}}\right) \\
& T_{\mathrm{c},-}^{\mathrm{irr}}=T_{\mathrm{c},-}+\left(15850 \mathrm{~K}-T_{\mathrm{c},-}\right) \frac{T_{\mathrm{irr}}^{2.1}}{T_{\mathrm{irr}, \mathrm{s}}^{2.1}} .
\end{aligned}
$$

For the viscous heating of the photosphere at the hot branch I find an expression similar to that of Cannizzo (1993a) and Ludwig \& Meyer (1998) also valid in the irradiated case:

$$
T_{\text {vis,hot }}=37346 \mathrm{~K} M_{\mathrm{wd}}^{-1 / 8} R_{10}^{3 / 8} T_{c, 5}^{2} \Sigma_{2}^{-1 / 2} .
$$

Here $\Sigma_{2} \equiv \Sigma / 100\left[\mathrm{~g} / \mathrm{cm}^{2}\right]$ and $T_{c, 5} \equiv T_{c} / 10^{5}[\mathrm{~K}]$. For the cold optically thick branch I obtain

$$
T_{\text {vis,cold }}=5500 \mathrm{~K}\left(\frac{\Sigma}{\Sigma_{-}}\right)^{0.17}\left(\frac{T_{\mathrm{c}}}{T_{\mathrm{c},-}}\right)^{0.41} R_{10}^{-0.05},
$$

where $\Sigma_{-}$is given by Eq. (7.7) and $T_{\mathrm{c},-}$ by Eq. (7.14) respectively.

For the unstable region between the critical values I use

$$
\log \left(T_{\mathrm{vis}}\right)=\frac{\log \left(T_{\mathrm{c}} / T_{\mathrm{c},+}\right)}{\log \left(T_{\mathrm{c},-} / T_{\mathrm{c},+}\right)} \log \left(\frac{T_{\mathrm{vis},+}}{T_{\mathrm{vis},-}}\right)+\log \left(T_{\mathrm{vis},+}\right)
$$




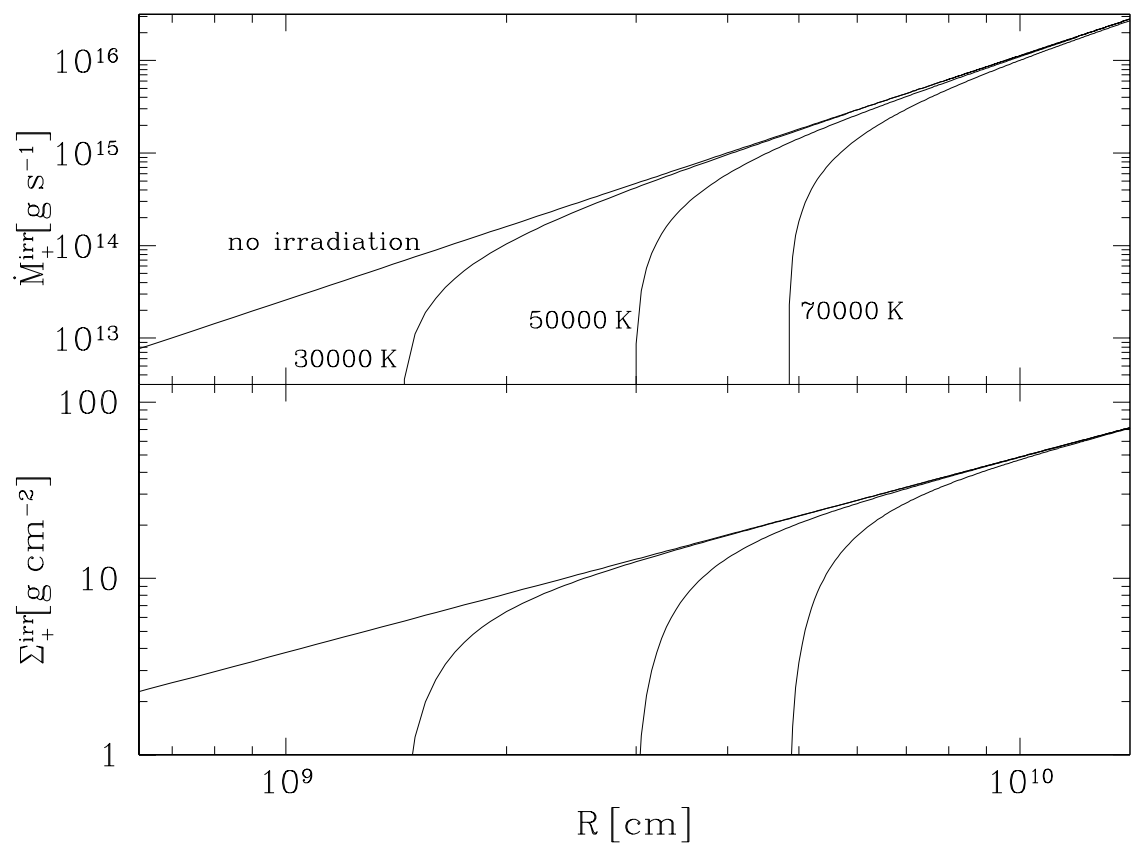

Figure 7.4: The critical accretion rate $\dot{M}_{+}^{\mathrm{irr}}$ and surface density $\Sigma_{+}^{\mathrm{irr}}$ (upper panel) in the inner disc regions for different amounts of irradiation: $(1-\beta)^{0.25} T_{\mathrm{wd}}=[30,50,70] \times$ $10^{3} \mathrm{~K}$. The used binary parameters correspond to the post nova system V446 Her, i.e. $R_{\text {out }}=3.8 \times 10^{10} \mathrm{~cm}, M_{\text {wd }}=0.8 M_{\odot}, R_{\text {in }}=6 \times 10^{8} \mathrm{~cm}$, and $\alpha_{\mathrm{h}}=0.1$.

and a sharp transition between $\alpha_{c}$ and $\alpha_{h}$ :

$$
\begin{aligned}
\log (\alpha)=\log \left(\alpha_{\mathrm{c}}\right) & +\left[\log \left(\alpha_{\mathrm{h}}\right)-\log \left(\alpha_{\mathrm{c}}\right)\right] \\
& \times\left[1+\left(\frac{T_{\mathrm{c}}}{0.5\left(T_{\mathrm{c},+}+T_{\mathrm{c},-}\right)}\right)^{8}\right]^{-1}
\end{aligned}
$$

similar to that used by Hameury et al. (1999).

\subsection{Dwarf nova outbursts of irradiated accretion discs}

Using the cooling functions, derived in the previous section, I can self-consistently simulate the behaviour of geometrically thin accretion discs in the vertical averaged description including external irradiation. In order to solve the equations of the time-dependent radial evolution of the disc I use the FE-code described in chapter 4 . 


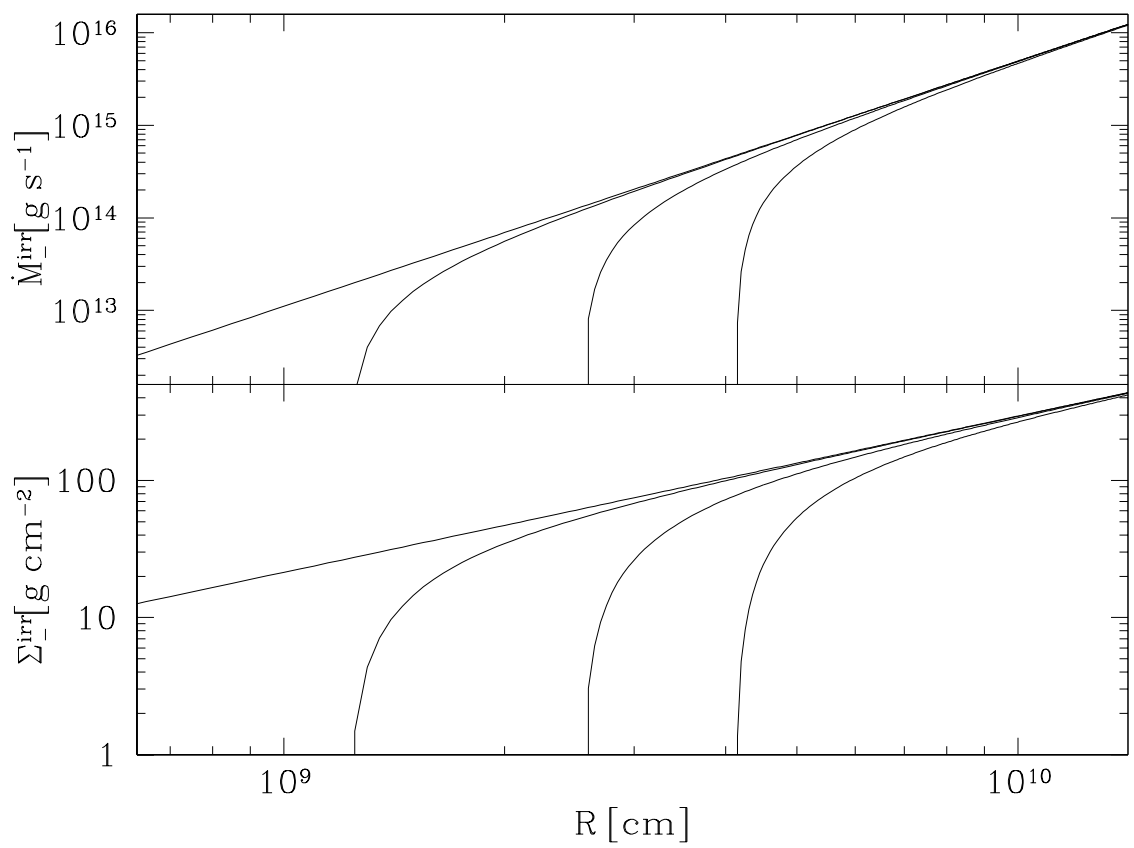

Figure 7.5: The same as in Fig. 7.4 for the critical values of the lower branch and $\alpha_{c}=$ 0.02 .

The irradiating flux from the white dwarf is given at each disc radius by:

$$
F_{\text {irr }}=(1-\beta) \frac{L_{\mathrm{wd}}}{2 \pi \sigma R_{\mathrm{wd}}^{2}} \frac{1}{\pi}\left[\arcsin \rho-\rho\left(1-\rho^{2}\right)^{\frac{1}{2}}\right],
$$

(Adams et al. 1988; King 1997). Here $R_{\mathrm{wd}}$ denotes the radius of the white dwarf, $\beta$ the albedo of the disc, $\rho \equiv R_{\mathrm{wd}} / R, L_{\mathrm{wd}}=4 \pi R_{\mathrm{wd}}^{2} \sigma T_{\mathrm{wd}}^{4}$ the luminosity of the white dwarf and $\sigma$ the Stefan-Boltzman constant.

In the following calculations I assume system parameters which are appropriate for the post nova V446 Her (Nova Herculis 1960), the best documented post nova that shows regular dwarf nova outbursts and that has a typical CV orbital period of $5 \mathrm{hr}$ (Honeycutt et al. 1998; Thorstensen \& Taylor 2000). Specifically, I use $R_{\text {out }}=3.8 \times 10^{10} \mathrm{~cm}, M_{\mathrm{wd}}=0.8 M_{\odot}, R_{\text {in }}=6 \times 10^{8} \mathrm{~cm}, \alpha_{\mathrm{h}}=0.1, \alpha_{\mathrm{c}}=0.02$, $\log \left(\dot{M}_{\mathrm{tr}}\left[\mathrm{g}, \mathrm{s}^{-1}\right]\right)=16.8$, and $d=1 \mathrm{kpc}$.

Figs. 7.4 and 7.5 show the effect of irradiation on the critical accretion rate and surface density $\left(\dot{M}_{+}^{\text {irr }}, \Sigma_{+}^{\text {irr }}, \dot{M}_{-}^{\text {irr }}, \Sigma_{-}^{\text {irr }}\right)$ for different temperatures of the white dwarf. I did not include the boundary layer luminosity in Eq. (7.21) because I am mainly interested in the effect of irradiation by a very hot white dwarf. The influence of both, the white dwarf and the boundary layer luminosity in the case of moderately 


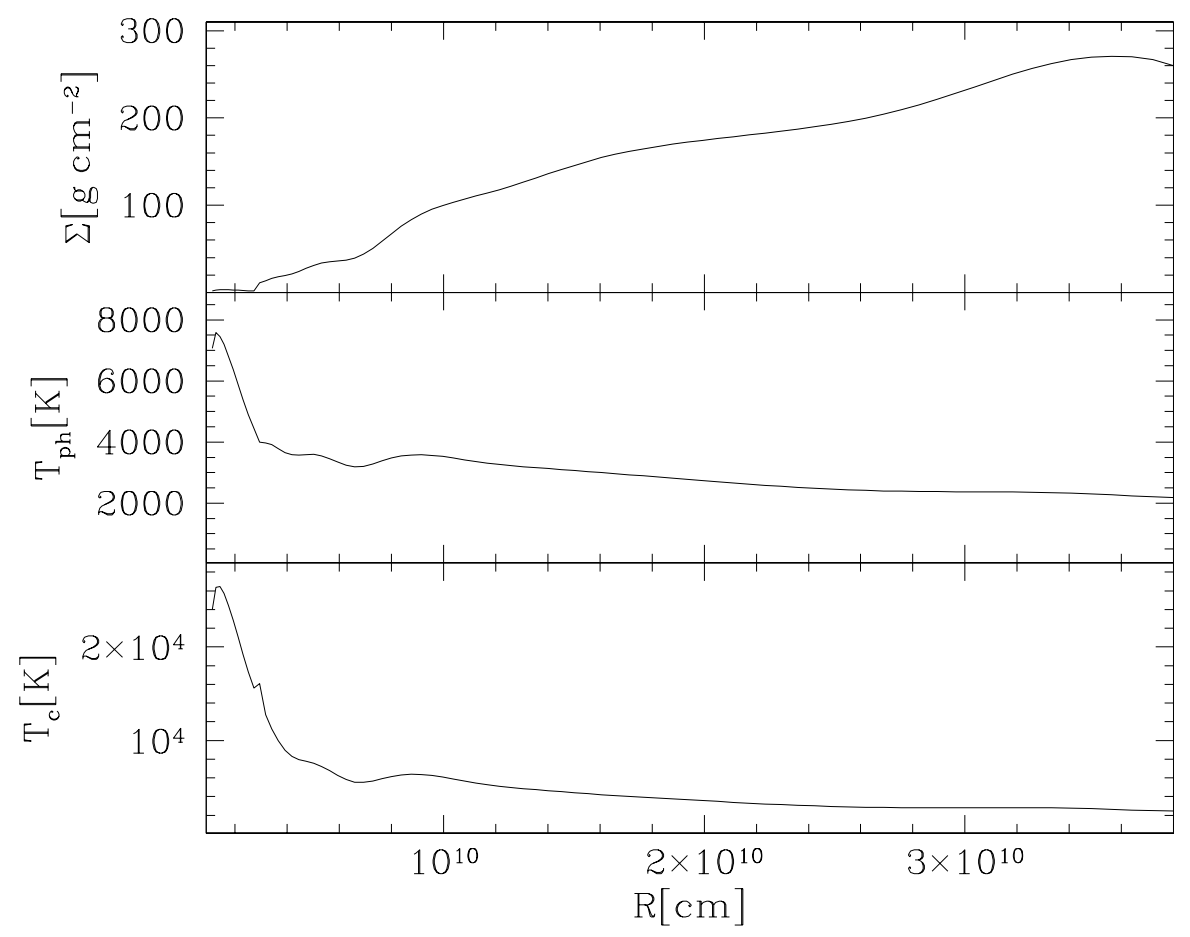

Figure 7.6: The radial structure of a disc irradiated by a white dwarf during quiescence. $T_{\mathrm{wd}}=30000 \mathrm{~K}$ and an albedo of $\beta=0.5$ are assumed. The innermost disc regions are depleted due to the strong irradiation flux. It is clear that there must exist a transition radius where the disc is unstable.

hot white dwarfs $(\sim 30000 \mathrm{~K})$ is discussed in Hameury et al. $(1999,2000)$.

The influence of irradiation by the white dwarf on the structure of the disc in quiescence is shown in Fig. 7.6. The strongly irradiated inner parts of the disc are kept in the hot state while the outer disc is cold and dense. The transition between the inner hot and the outer cool regions is necessarily unstable.

Fig. 7.7 shows light curves for different amounts of disc irradiation. To determine the visual brightness $V$, I use the prescription of Cannizzo (1993a) but scale for the larger distance and I used $T_{\mathrm{ph}}^{4}=T_{\mathrm{vis}}^{4}+T_{\mathrm{irr}}^{4}$ instead of his $T_{\mathrm{eff}}^{4}$.

The obtained results confirm the finding of Hameury et al. (1999), i.e. that irradiation causes small post-eruption outbursts. These outbursts occur because the cooling wave gets reflected when reaching the inner parts of the disc where the instability is suppressed. This clearly shows that there is an unstable transition region in the disc which causes heating and cooling fronts travelling through the disc producing small outbursts. In the light curves of Fig. 7.7 most of these small outbursts can not be discerned because they affect only a small area of the disc and do not contribute much to the overall brightness of the strongly irradiated disc. 


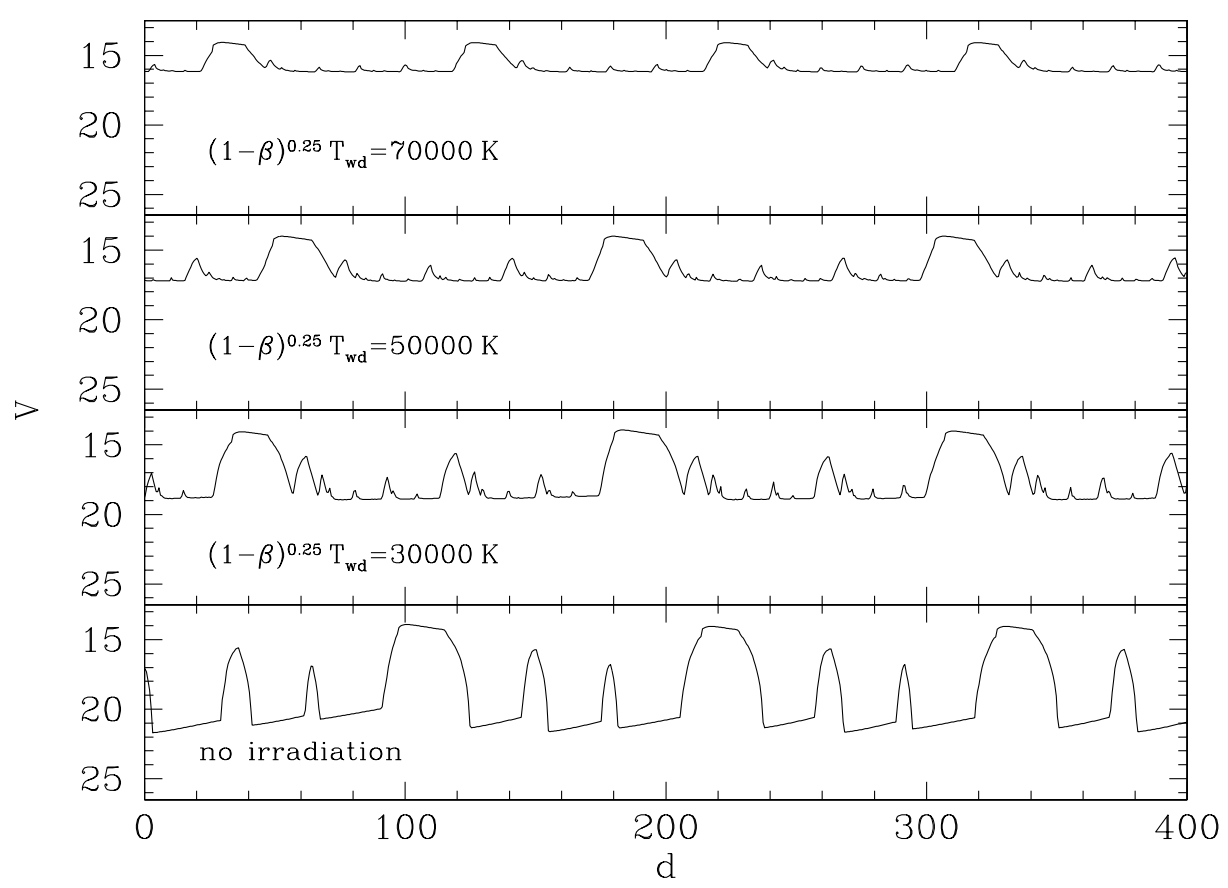

Figure 7.7: Light curves for different amounts of irradiation using system parameters appropriate for V446 Her. Because of the irradiation, there is always an unstable region in the disc causing small "echo" outbursts immediately following normal outbursts. In the case of very strong irradiation (upper panel) these small outbursts are hardly seen because the visual brightness of the irradiated disc outshines most of these small outbursts.

The light curve obtained for $(1-\beta) T_{\mathrm{wd}}=30000$ (third panel from top in Fig. 7.7) is very similar to the light curve presented by Hameury et al. (1999) (upper panel of their Fig. 3) using $M_{\mathrm{wd}}=0.6$ and low mass transfer rates.

The appearance of several small outbursts between the major ones contrasts with the observed dwarf nova light curves. For example, SS Cygni, the dwarf nova which has become the standard system for light curve analysis, contains a relatively hot white dwarf, $T_{\mathrm{wd}}=3-4 \times 10^{4} \mathrm{~K}$ (Warner 1995), but there never were observed "echo" outbursts following the major ones.

There exist at least three possibilities to explain this disagreement between calculated and observed light curves:

- the inner parts of the disc, which would be mostly affected by irradiation, are emptied by evaporation.

- the albedo $\beta$ is large and the fraction of the illumination flux which penetrates the discs photosphere is significantly smaller than previously esti- 
mated.

- the disc limit-cycle model in the present form is not the appropriate explanation of dwarf nova outbursts.

To make a decision between the possibilities above nature provided us with a system in which an outbursting accretion disc is strongly and time-dependently irradiated by the white dwarf.

\subsection{Dwarf novae among post novae}

As noted in chapter 2, nova eruptions and dwarf nova outbursts may resemble each other in some cases. In fact, a number of large amplitude/low outburst frequency dwarf novae are found in the lists of nova remnants, the most famous example being the short-period dwarf nova WZ Sge (Duerbeck 1987). In spite of that, the physical mechanisms behind the outbursts are entirely different (see chapter 2 and 4)

It is clear that the class of cataclysmic variables (CVs) provides numerous potential novae progenitors (pre-novae). In fact, almost all CVs should suffer nova eruptions repeatedly during their lives. Up to now, no system became a nova after it had been classified as a CV. However, a large number of nova remnants (post novae) have been found to be CVs after they attracted attention by erupting (novae may arise, of course, on a white dwarf accreting hydrogen-rich material in other environments, e.g. in a symbiotic binary). Among the wide variety of known CV subtypes, most post novae were found to have rather high mass transfer rates $\dot{M}$, and, thus, fall into the class of nova-like variables. It is interesting to note that the published mass transfer rates were generally determined under the assumption that the disc luminosity arises only from viscous dissipation although irradiation of the disc by the hot white dwarf contributes significantly to the observed disc luminosity in "young" post novae.

Nevertheless, the mass transfer rates in most post novae are indeed relatively high because only a few of them show dwarf nova outbursts. Warner (1995) finds in his sample of sixty well-observed post novae (obtained from the sources: Duerbeck 1987; Warner 1987; van den Bergh \& Younger 1987; Harrison \& Gehrz 1988, 1991; Gaposchkin 1957; Downes \& Shara 1993) only five post novae which show dwarf nova outbursts after the eruption: GK Per, V1017 Sgr, Q Cyg, WY Sge, V446 Her. GK Per and V1017 Sgr have very long orbital periods (2 and $5.7 \mathrm{~d}$ (Warner 1995; Sekiguchi 1992)) and the dwarf nova nature of WY Sge and Q Cyg is uncertain (Somers et al. 1996; Shugarov 1983). Hence, V446 Her (Nova Her 
1960) is up to now the only normal dwarf nova among post novae and it is clear that most post novae have mass transfer rates too high to permit dwarf nova outbursts.

Even though not directly related to the accretion disc, the nova event 40 years ago has deep implications for the disc surrounding the white dwarf in V446Her: in the nova remnant, or post nova, the white dwarf heated during the nova eruption (see section 2.3) slowly cools down and intensively irradiates the accretion disc with a dramatic impact on the structure of the disc.

In the following sections, I analyse the circumstances under which a post nova will evolve into a dwarf nova following the nova eruption. First, I calculate the irradiating flux of the accretion disc by the hot white dwarf, and estimate the amount of time, required for a post nova to cool to the point when it may begin exhibiting dwarf nova outbursts. Thereafter I discuss the results in the context of the post nova with the best observational coverage to test the obtained results - V446 Her.

\subsubsection{White dwarf cooling in post novae}

Even though a large part of the accreted hydrogen-rich material is ejected during the nova eruption, a significant amount of hydrogen is left over in the remaining envelope which rapidly returns to hydrostatic equilibrium (MacDonald 1996). The nova remnant experiences steady-state hydrogen shell burning until the nuclear fuel is exhausted, and is consequently heated to effective temperatures of several $10^{5} \mathrm{~K}$. The turn-off times of the burning envelope, which have been observed with ROSAT for a small number of novae are of the order of years, e.g. $1.8 \mathrm{yr}$ in V1974 Cyg (Krautter et al. 1996) and $10 \mathrm{yr}$ in GQ Mus (Shanley et al. 1995). Additional support for turn-off times on the order of years comes from monitoring of the ultraviolet luminosity of a number of post novae following their eruption (Gonzalez-Riestra et al. 1998).

Prialnik (1986) modelled the evolution of a classical nova through a complete cycle of accretion, outburst, mass loss, decline and resumed accretion. During the decline, the cooling of the white dwarf can be fit with a power-law of the form

$$
L \propto t^{-1.14}
$$

where $L$ denotes the luminosity of the white dwarf and $t$ the time after the nova explosion. Prialnik's theoretical model is confirmed by Somers \& Naylor (1999), who derive the cooling rate of the white dwarf in V1500 Cygni (Nova 1975 Cygni) using $\mathrm{B}$ band observations of the irradiated secondary and find

$$
L \propto t^{-0.94 \pm 0.09}
$$




\subsubsection{Time-dependent disc irradiation}

It is clear that the obtained cooling law for the white dwarf in post novae Eq. (7.22) leads to time-dependent irradiation of the accretion disc by replacing the constant luminosity of the white dwarf in Eq. (7.21) by the power law given in Eq. (7.23).

The luminosity of the white dwarf is given by

$$
L_{\mathrm{wd}}(t)=4 \pi R_{\mathrm{wd}}^{2} \sigma T_{\mathrm{wd}}^{4}(t) \propto t^{-1.14},
$$

where $R_{\mathrm{wd}}$ and $T_{\mathrm{wd}}$ denote the radius and the effective temperature of the white dwarf respectively, and $\sigma$ is the Stefan-Boltzman constant. In the calculations I present in section 7.3.3 I additionally consider a boundary layer luminosity

$$
L_{\mathrm{BL}}=\alpha_{\mathrm{BL}} \frac{G M_{\mathrm{wd}} \dot{M}}{R_{\mathrm{wd}}},
$$

where $G$ is the gravitational constant and, as in Stehle \& King (1999) and Leach et al. (1999) I take $\alpha_{\mathrm{BL}}=0.5$. Assuming that the boundary layer luminosity is radiated by the entire surface of the white dwarf and that the disc is geometrically thin, the time-dependent flux $F_{\text {irr }}(t)$ irradiating the disc at the radius $R$ is given by

$$
F_{\text {irr }}(t)=(1-\beta) \frac{L_{\mathrm{BL}}+L_{\mathrm{wd}}(t)}{2 \pi \sigma R_{\mathrm{wd}}^{2}} \frac{1}{\pi}\left[\arcsin \rho-\rho\left(1-\rho^{2}\right)^{\frac{1}{2}}\right],
$$

where $\beta$ is the albedo, $\rho=R_{\mathrm{wd}} / R$ and $t=0$ at the end of the hydrogen shell burning phase.

\subsubsection{Irradiation limits on the occurrence of dwarf novae in post novae}

If the irradiation temperature $\left(\sigma T_{\text {irr }}^{4} \equiv F_{\text {irr }}\right)$ exceeds $T_{\text {irr,s }}$ given in Eq. (7.8) the hydrogen in the disc is fully ionised independent on the accretion rate. Thus, setting $T_{\text {irr }} \geq T_{\text {irr,s }}$ at the outer edge of the disc $\left(R=R_{\text {out }}\right)$ gives a limit of the irradiation flux which suppresses the disc instability for the entire disc. Using standard equations (Eggleton 1983; Frank et al. 1992) and assuming that the outer disc radius $R_{\text {out }}$ is $70 \%$ of the primary's Roche lobe radius, I obtain the outer radius of the accretion disc as a function of the orbital Period $P$ and the binary mass ratio $q=M_{2} / M_{\mathrm{wd}}$. I use $q=0.5$ for all calculations throughout this section as I am mainly interested in systems above the period gap.

Now it is possible to estimate how long disc instabilities in the accretion discs of post novae are suppressed due to irradiation. Assuming either $\dot{M}=0$ or $\dot{M}=$ 


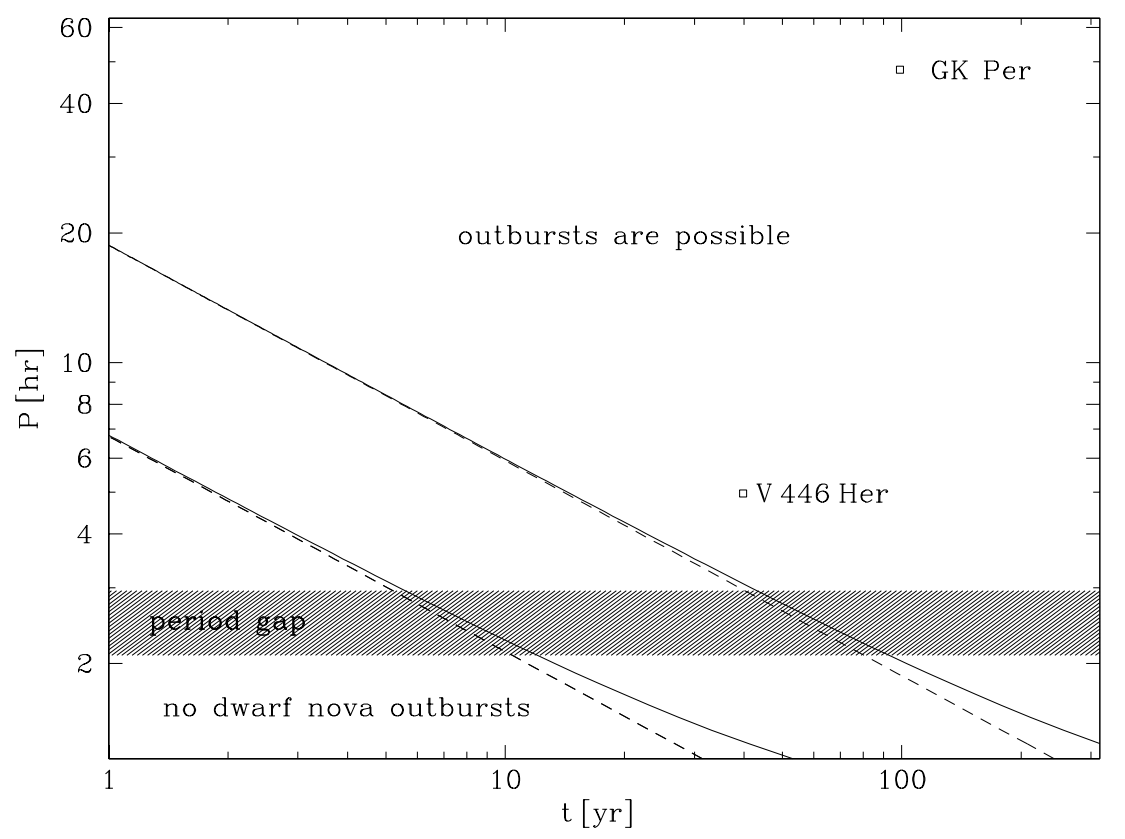

Figure 7.8: Irradiation limits on the occurrence of dwarf nova outbursts as a function of time after the nova eruption and orbital period. I use initial effective temperatures $T_{\mathrm{wd}}=$ $5 \times 10^{5} \mathrm{~K}$ (upper curves) and $T_{\mathrm{wd}}=3 \times 10^{5} \mathrm{~K}$ (lower curves). The solid lines represent calculations including a boundary layer luminosity corresponding to $\dot{M}_{\mathrm{wd}}=10^{17} \mathrm{~g} \mathrm{~s}^{-1}$, whereas the dashed lines are calculated using only the luminosity of the cooling white dwarf. Note that $t=0$ corresponds to the end of the hydrogen shell burning phase, which might last for up to $\sim 10 \mathrm{yr}$ after the actual nova eruption.

$10^{17} \mathrm{~g} \mathrm{~s}^{-1}$, as typical for dwarf novae above the period gap (for much higher accretion rates, the disc remains in a stable, hot state anyway), Fig. 7.8 shows the results for initial temperatures of $T_{\mathrm{wd}}(0)=5 \times 10^{5} \mathrm{~K}$ and $T_{\mathrm{wd}}(0)=3 \times 10^{5} \mathrm{~K}$. Apparently, disc instabilities and, hence, dwarf nova outbursts should typically be suppressed for $\sim 5-50 \mathrm{yr}$, and up to $\sim 100 \mathrm{yr}$ if the white dwarf is heated to very high temperatures during the nova eruption. It is also clear that the size of the disc is a crucial parameter: the irradiation from the white dwarf can suppress the instability over the entire disc only for orbital periods $P_{\text {orb }} \leq 20 \mathrm{hr}$. It is therefore not surprising that GK Per (orbital period $\sim 2 \mathrm{~d}$ ) was not observed in a persistent, nova-like state.

The contribution of the boundary layer luminosity becomes important only after $\sim 5 \mathrm{yr}$ at the earliest.

It is important to note that the results presented in Fig. 7.8 are lower limits on 
the time scale on which irradiation from the white dwarf suppresses dwarf nova outbursts in post novae for the given disc albedo: (a) in order to produce significant dwarf nova outbursts, the disc instability has to affect on considerable parts of the outer disc and not only the outer edge (I focus on this subject in the next section) and (b) the disc may be flared and therefore intercept more flux from the white dwarf than described by Eq. (7.26) which assumes a flat disc.

\subsubsection{Detailed long term light curves of post novae}

Using the cooling functions derived in section 7.1 I calculate the time dependent evolution of irradiated discs in post nova. Eqs. (7.22) and (7.26) describe the time-dependent irradiating flux. Assuming a disc albedo of $\beta=0.5$ I considered the following three cases.

(a) I used the system parameters of V446 Her (see section 7.2 and Fig. 7.4) and a turn-off temperature of $3 \times 10^{5} \mathrm{~K}$. Even for such a conservatively low turn-off temperature, the white dwarf in V446 Her is today - 40 years after the nova explosion - still very hot, $\sim 1.2 \times 10^{5} \mathrm{~K}$. The simulated light curve displayed in Fig. 7.9 \& Fig. 7.10 (a) qualitatively reproduces the observations of Stienon (1971) and Honeycutt et al. (1998): between September 1968 and September 1970 the system was observed several times at $m_{\mathrm{pg}} \sim 15.8$ and in 1998 regular dwarf nova outbursts of 2.5 magnitudes ( $V$ between 18 and 15.5) were observed.

Considering the relatively poor sampling of the observed light curve of V446 Her and the uncertainties in the turn-off time, in the system parameters $\left(M_{\mathrm{wd}}, M_{\mathrm{sec}}\right.$, $\dot{M}_{\text {tr }}, d$ ), and, most important, in the disc albedo $\beta$, I did not attempt to quantitatively fit the actual magnitude and outburst frequency of V446 Her. However, once that detailed observed light curves of V446 Her that resolve the actual shape of the dwarf nova outbursts are available, such a quantitative comparison will be a crucial test for the theory of the thermal instability in irradiated accretion discs and a promising way to estimate the efficiency of disc irradiation, i.e. $\beta$. Note that even if the reprocessing efficiency is as low as e.g. the calculations by Suleimanov et al. (1999) suggest (they find $\beta \sim 0.93$ for irradiated accretion discs in super soft $\mathrm{X}$-ray sources), irradiation by the hot white dwarf in V446 Her should still noticeably influence the outburst behaviour in this system. For $\beta=0.93$ and an actual white dwarf temperature of $T_{\mathrm{wd}}=1.2 \times 10^{5} \mathrm{~K}$, the corresponding "effective irradiation" temperature is

$$
(1-\beta)^{0.25} T_{\mathrm{wd}} \simeq 50000 \mathrm{~K}
$$

and the resulting light curve contains observable echo outbursts (Fig. 7.7, second panel from top). 

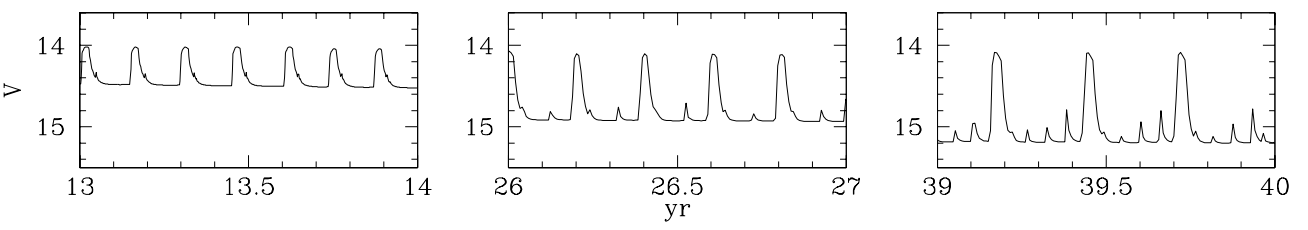

Figure 7.9: Snapshots of dwarf nova outbursts from the long-term simulation shown Fig. 7.10 (a). The the left panel corresponds to $T_{\mathrm{wd}} \simeq 1.6 \times 10^{5} \mathrm{~K}$ and the outburst magnitude is only 0.5 magnitudes. The cooling wave gets repeatedly reflected between the hot inner part of the disc and the disc edge, resulting in an exponential decline superimposed by small dips. After 26 years the white dwarf has cooled down to $\simeq 1.3 \times 10^{5} \mathrm{~K}$ and small echo outburst appear between the larger ones. Today ( $\sim 40 \mathrm{yr}$ after turn-off), $T_{\mathrm{wd}}=1.2 \times 10^{5} \mathrm{~K}$ and the small outbursts, caused by multiple reflections of the heating and cooling waves, become increasingly pronounced.

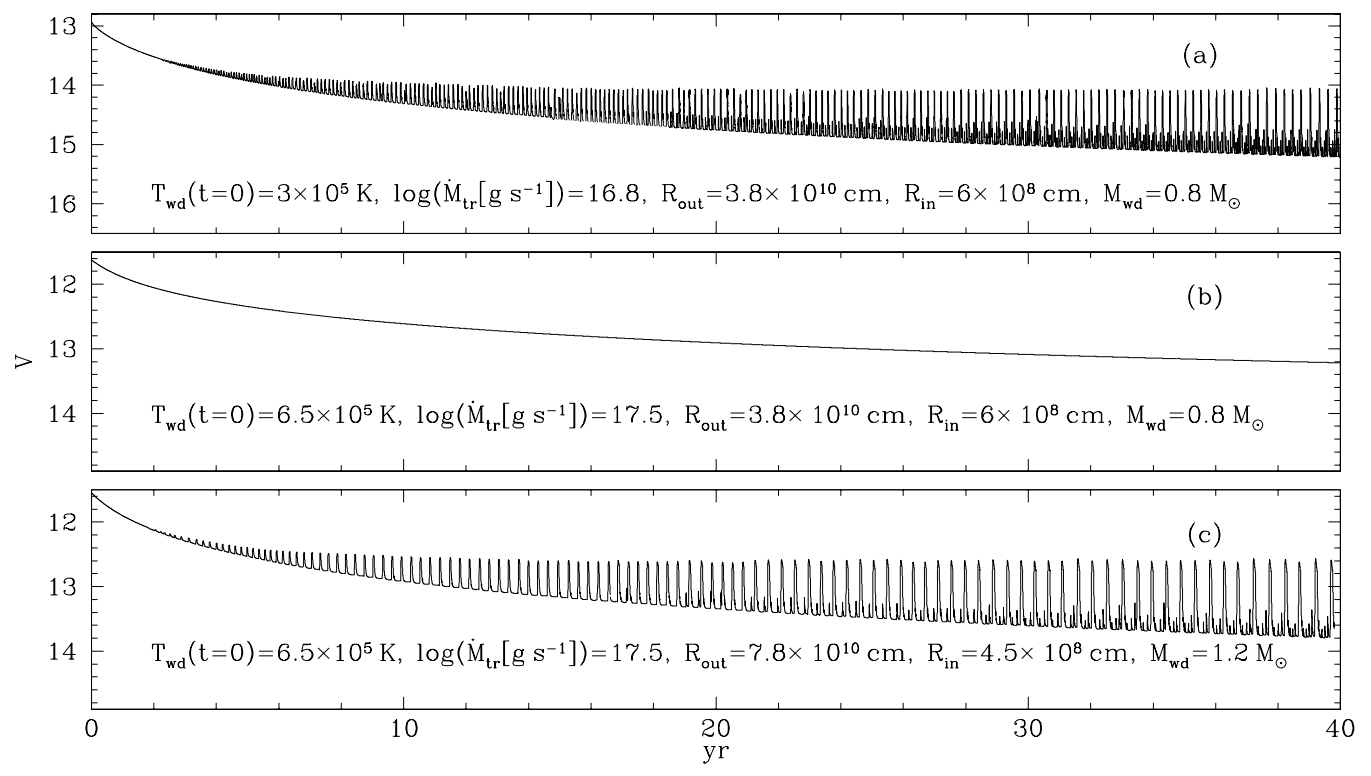

Figure 7.10: Simulations of the occurrence of dwarf nova outbursts in post novae assuming moderate and constant mass transfer, a disc albedo of $\beta=0.5, \alpha_{h}=0.1, \alpha_{c}=0.02$ and $L_{\mathrm{wd}} \propto t^{-1}$ as cooling law for the white dwarf (see Eq. (20)). Panel (a) displays the light curve most realistic for V446 Her: the outbursts start $\sim 5 \mathrm{yr}$ after the hydrogen burning turned off and the magnitude of the outbursts today 1960+40 is around 1 mag. Panel (b) displays the most extreme case of the calculations: I assumed a relatively small disc and a turn-off temperature $T_{\mathrm{wd}}(t=0)=6.5 \times 10^{5} \mathrm{~K}$. As a result, the thermal instability is suppressed for a few decades. The influence of the disc radius (i.e., the orbital period) is shown in panel (c): even in the case of an extremely heated white dwarf and a high accretion rate, such a large disc produces outbursts already $\sim 5 \mathrm{yr}$ after the hydrogen burning turned off. 


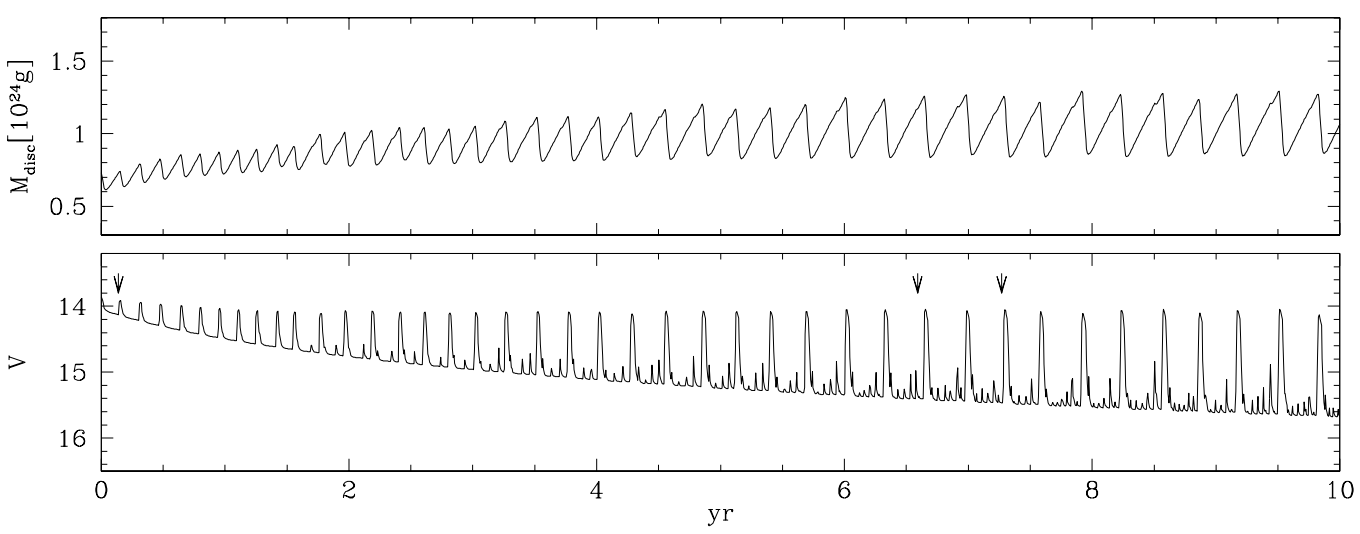

Figure 7.11: The evolution of the disc mass and the light curve for ten years after the nova eruption. The parameters are the same as in Fig. 7.10 (a) except the disc albedo which is set to $\beta=0.93$. The outer regions of the disc are affected by the instability and show a limit-cycle behaviour. The upper panel displaying the disc mass clearly shows that the small outbursts between the major ones have negligible influence on the disc mass. The structure of the disc for the times marked with arrows are shown in Figs. 7.12-7.14.

(b) I used again the system parameters of V446 Her, but a much higher turn-off temperature of $T_{\mathrm{wd}}(t=0)=6.5 \times 10^{5} \mathrm{~K}$ and significantly higher mass transfer, $\log \left(\dot{M}_{\mathrm{tr}}\left[\mathrm{g} \mathrm{s}^{-1}\right]\right)=17.5$ instead of $\log \left(\dot{M}_{\mathrm{tr}}\left[\mathrm{g} \mathrm{s}^{-1}\right]\right)=16.8$ to calculate the visual decline and the suppression of disc outbursts under extreme conditions as shown in Fig. 7.10 (b). In this case the suppression of disc instabilities due to irradiation lasts many decades.

(c) The third simulation shows the light curve produced by a larger accretion disc $\left(R_{\text {in }}=7.8 \times 10^{10} \mathrm{~cm}\right)$ around a more massive but again very hot primary $\left(M_{\mathrm{wd}}=1.2 M_{\odot}, T_{\mathrm{wd}}(t=0)=6.5 \times 10^{5} \mathrm{~K}\right)$, corresponding to a system with a long orbital period, $P \sim 15 \mathrm{hr}$ (Fig. 7.10(c)). The occurrence of dwarf nova outbursts is prevented for only $\sim 5 \mathrm{yr}$ although I assumed an extremely high turn-off temperature of the white dwarf. For CVs having a long orbital period, the strong disc irradiation after a nova eruption can hardly suppress disc instabilities in the entire disc. An example of such a system is GK Per (Nova Per 1901) which was never detected as a persistent nova-like system. In addition Fig. 7.10 (b) shows that the duration of the outbursts and the quiescence intervals are longer than for smaller discs, as expected.

Considering the uncertainties in $\beta$, I recalculate the first ten years of case (a) using $\beta=0.93$ as derived by Suleimanov et al. (1999) in a study of irradiated accretion discs in super soft x-ray sources. Fig. 7.11 shows the resulting light curve and the evolution of the disc mass. The disc starts producing outbursts immediately 


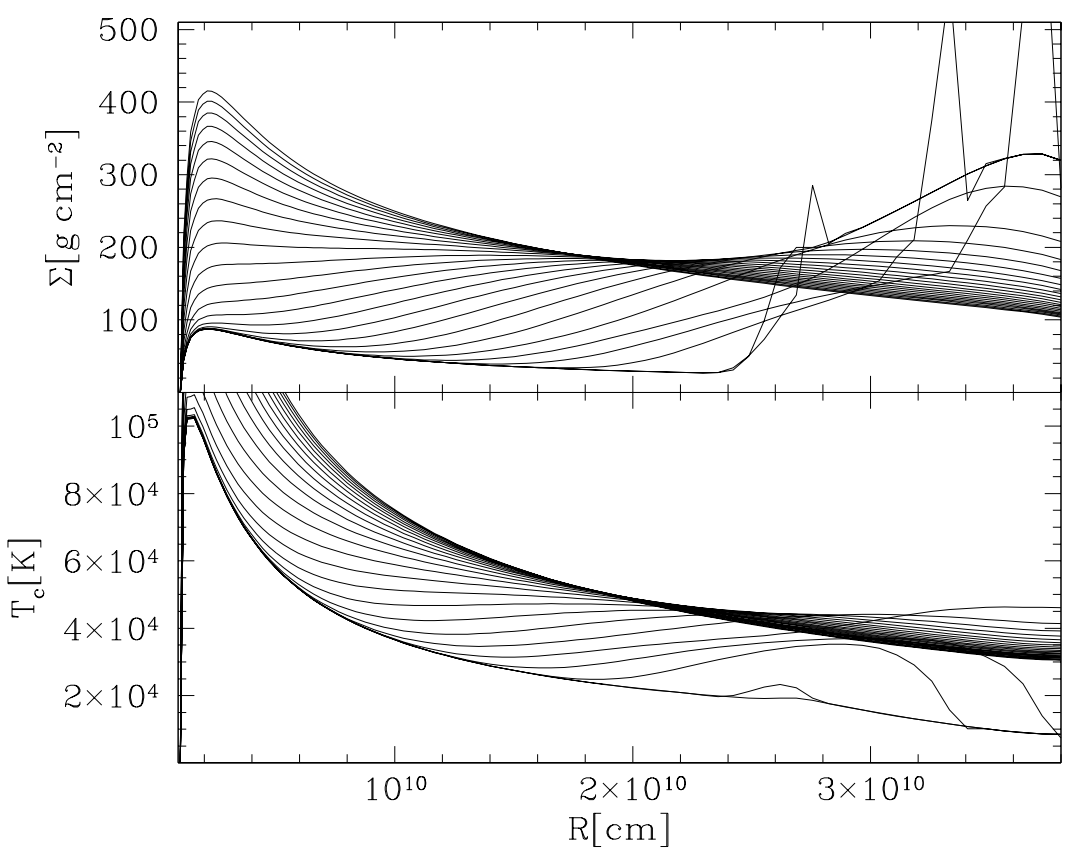

Figure 7.12: The radial structure during the first outburst of the light curve in Fig. 7.11. The time-steps between the lines are $\Delta t=0.23$ days. The disc is strongly heated and kept in the hot state for every disc annulus inside $R=2.6 \times 10^{10} \mathrm{~cm}$ because the temperature of the white dwarf is $\sim 2.90 \times 10^{5} \mathrm{~K}$ whereas the outer disc goes through a limit-cycle behaviour. After the surface density at the transition radius has reached the critical value given by Eq. (7.12) a heating front starts and brings the entire disc into the hot state.

after the hydrogen burning turned off. The magnitude of the outbursts is $\sim 1.5 \mathrm{mag}$ already ten years after the turn off whereas in Fig. 7.10(a) the outburst magnitude reaches roughly 1 mag even 40 years after eruption. Considering that Honeycutt et al. (1998) obtained brightness variations of $\sim 2.5 \mathrm{mag}$, this suggests a rather high value for the disc albedo.

In Fig. 7.10 as well as in Fig. 7.11 the outburst magnitude is increasing with time whereas the outburst frequency decreases. This is a result of the continuous cooling of the white dwarf as the decreasing irradiation of the accretion disc allows an increasing angular extent of the accretion disc to participate in limit-cycle oscillations.

Fig. 7.9-7.11 display the light curves of strongly irradiated discs but thus far I have not shown the structure and the dynamical behaviour of such discs. Fig. 7.12 shows the propagation of the heating front during the first dwarf nova outburst after the nova eruption. The disc is strongly heated and kept in the hot state for 


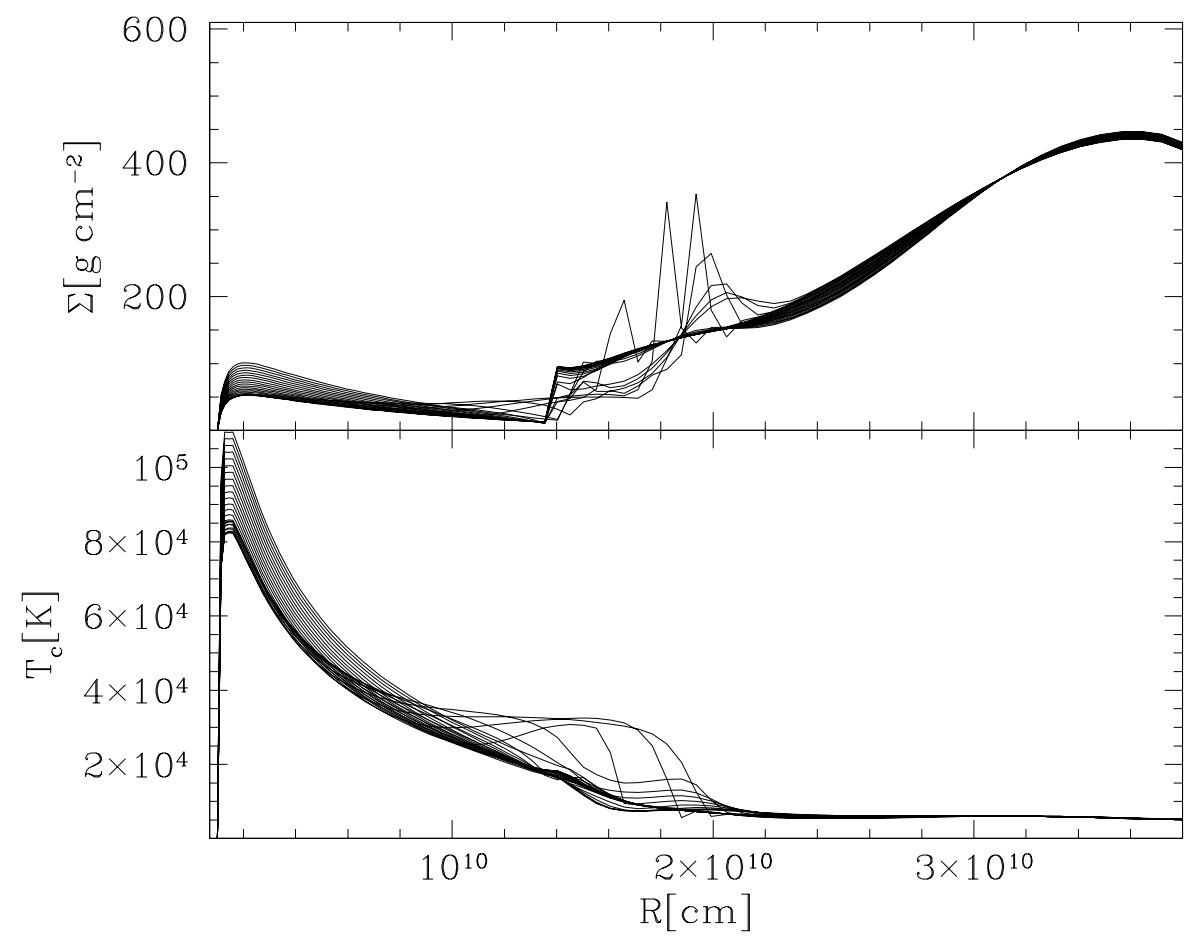

Figure 7.13: The dying heating front of the small outburst $\sim 6.55$ years after turn off (see Fig. 7.11). The time-steps between the lines are $\Delta t=0.23$ days as in Fig. 7.12. As there is not enough mass in the outer disc the heating front gets reflected before it has reached the outer edge of the disc. Such small outbursts are necessarily present in an efficiently irradiated disc as every heating front gets reflected when reaching the inner hot regions of the disc. The white dwarf temperature for this snapshot is $\sim 1.8 \times 10^{5} \mathrm{~K}$.

every disc annulus inside $R=2.6 \times 10^{10} \mathrm{~cm}$. After a heating front switches the outer dense disc into the hot state, the disc is in the quasi-stationary outburst state. In Fig. 7.13 it is shown how the disc behaves during a small outburst. As there is not enough mass stored in the outer disc the heating front "dies" before it reaches the outer edge of the disc. For completeness, I display in Fig. 7.14 the rise to a major outburst $\sim 7.5$ years after the hydrogen burning turned off.

\subsection{Conclusion}

I derived approximations for the cooling law of irradiated accretion discs using detailed calculations of the vertical structure. This makes it possible to selfconsistently calculate the time-dependent behaviour of irradiated discs in the ver- 


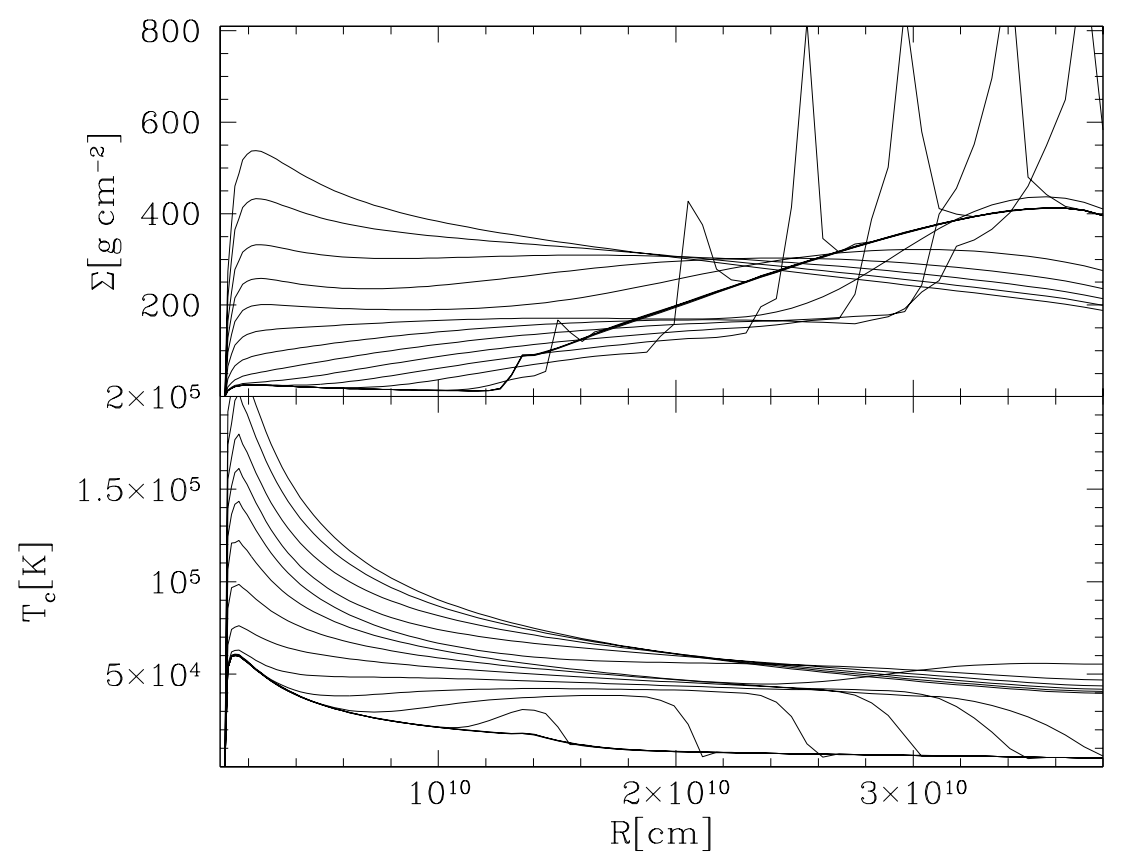

Figure 7.14: After a few small outbursts the outer disc contains enough mass for a major outburst. The time-steps between the lines are $\Delta t=0.46$ days. The white dwarf has continuously cooled down to $\sim 1.77 \times 10^{5} \mathrm{~K}$. This snapshot corresponds to the large outburst at $t=7.5$ years in Fig. 7.11 (third arrow).

tically averaged description.

I showed that irradiation of the accretion disc by the white dwarf in dwarf nova systems causes the appearance of "echo" outbursts immediately following the bigger outbursts. This contrasts the observed light curves of dwarf nova if one assumes the irradiation to be rather efficient as suggested by King (1997). It is important to note, that the occurrence of "echo" outbursts (most evident in Fig. 7.7, third panel from above) does not depend on the assumed viscosity. It reflects the unavoidable presence of a transition region between hot and cold regimes in quiescence. The detailed light curve still depends on the viscosity and hence on assumed value of $\alpha$ but the general appearance of the small outbursts following immediately the larger ones does not.

I further investigated on disc irradiation in post novae, where the white dwarf is much hotter than in normal dwarf novae. I derived irradiation limits for the occurrence of dwarf nova outbursts in post novae and showed that these limits as well as the calculated detailed light curves of post novae are in agreement with the photometric history of V446 Her, the most appropriate system to test the predictions 
of the theory of irradiated discs.

I have calculated the effect that irradiation by the heated white dwarf in a post nova has on the structure of an accretion disc and I found that, even if the accretion rate in such a system is low enough to permit disc instabilities, irradiation from the white dwarf suppresses dwarf nova outbursts for up to $\sim 100 \mathrm{yr}$. In the case of V446 Her the calculations predict an increase of the outburst amplitude and a decrease of the outburst frequency as the white dwarf keeps on cooling down.

Both, the prediction of "echo" outbursts in normal dwarf novae and the calculation of post novae discs indicate that the efficiency of irradiation by the white dwarf is probably rather small. Although the results obtained from simulations of irradiated discs in post novae are in good agreement with the observations of V446 Her, the overall observational situation is rather unsatisfactory. To my knowledge no "echo" outbursts have ever been observed in CVs; neither in normal dwarf novae nor in post nova systems. If "echo" outbursts are observed in a system with strong disc irradiation, this would verify the thermal limit-cycle model in a unique and $\alpha$-independent way.

In order to relate the theory of irradiated accretion discs presented here with observations it is of great importance to obtain long term monitoring of V446 Her. 


\section{Chapter 8}

\section{Future targets: the fortunate case of V 446 Her}

In chapter 7 I presented self-consistent calculations of irradiated accretion discs around white dwarfs, especially in post novae. Although the theoretical background of irradiated accretion discs is well developed, there is no clear observational evidence that in nature irradiation does stabilise disc accretion and the efficiency of reprocessing in the disc is rather uncertain.

Fortunately, there exists a quite unique system to test time-dependent models of irradiated accretion discs with: V446 Her (Nova Her 1960) developed regular dwarf nova outbursts $\sim 30$ years after its nova eruption in 1960 (Honeycutt et al. 1995, 98). Although it is certain that the white dwarf in V446 Her is extremely hot, the estimate of the white dwarf temperature $T_{\mathrm{wd}}$ used in chapter 7 are somewhat rough because both the turn-off temperature and the duration of the hydrogen burning phase are unknown for V446 Her. A clear prediction of my calculations is, that V446 Her shows "echo" outbursts and additional small outbursts between large outbursts.

Therefore, I intend to improve on the unclear observational situation and present here preliminary results from an observing campaign on V446 Her.

\subsection{Observations}

In order to obtain a detailed light curve of V446 Her I started an intensive VSNET (Variable Star NETwork) campaign. Unfortunately it is difficult to observe V446 Her for most of the amateur observers, as V446 Her is rather faint and a close optical triple. Depending on the seeing conditions, the three stars can not be 


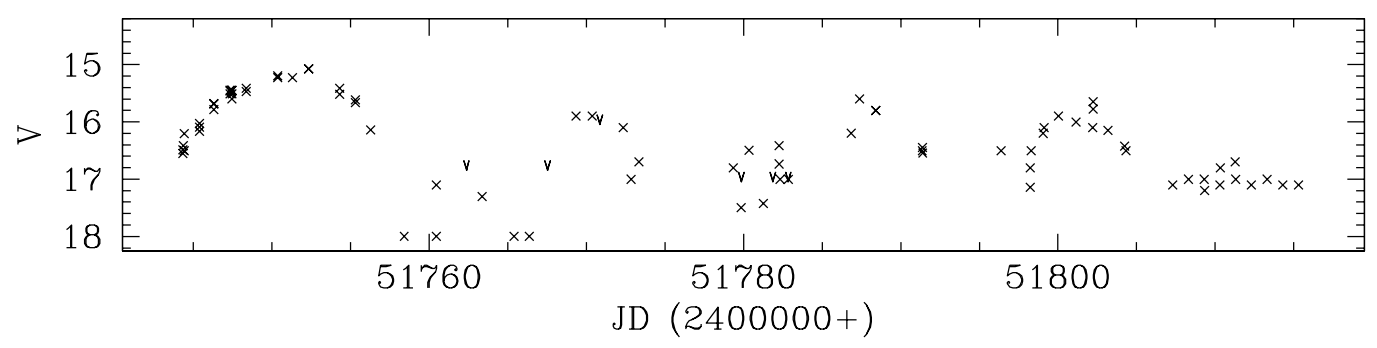

Figure 8.1: Preliminary light curve of V 446 Her obtained by a VSNET campaign. There are clear irregular brightness fluctuations following the outburst at JD=51760 which may reflect the theoretical predicted "echo" outbursts.

resolved.

However, a few well equipped astronomers joined the campaign from August until October 2000. The resulting light curve is shown in Fig. 8.1. After one clear outburst the system goes through some irregularities which could be interpreted as "echo" outbursts. Unfortunately, V446 Her is situated too close to the sun since October and the campaign came to an end.

The light curve presented in Fig. 8.1 is somewhat preliminary as I did not yet receive all the CCD images from the campaign. A systematic analysis of these images will have to deal with the possible overlap of the two nearby stars. If the indications for "echo" outbursts shown in Fig. 8.1 can be confirmed by further observations, the developed theory of irradiated accretion disc will be supported in a unique way.

\subsection{Proposed observations: detecting the hottest white dwarf in a dwarf nova}

In addition to the VSNET campaign I proposed Hubble Space Telescope observations of V446 Her in order to obtain high-quality STIS/FUV spectroscopy, which will very likely reveal that V446 Her is the dwarf nova containing the hottest white dwarf. With these observations in hand, it will be possible to accurately determine the white dwarf temperature.

I will use the derived temperature to calculate the disc irradiation in V446 Her, thus allowing a more quantitative comparison of observed and calculated light curves (Figs. 7.7, 7.10, and 7.11). The presented state-of-the-art finite-element 
disc evolution code is - apart from that of Hameury et al. (1999) - the only one that can self-consistently treat irradiation of the disc. I will use this code and the white dwarf temperature obtained from the HST observations of V446 Her to calibrate the efficiency of reprocessing in the accretion disc photosphere. This will determine the dynamical importance of disc irradiation in general. Subsequently, I will be possible to carry out the first time-dependent calculations of a strongly irradiated disc in which the amount of irradiation is based on hard observational facts. 


\section{Chapter 9}

\section{Implications: a new post nova scenario?}

So far I have only "used" the post nova system V446 Her to learn from it about the physics of irradiated accretion discs. Now it is time to return some efforts to this very interesting system. As disc irradiation is very strong in post novae it provides deep implications for the evolution of post novae and, hence, for the CV evolution in general.

\subsection{The hibernation scenario for post novae}

The physics of nova eruptions are reviewed in section 2.3. As noted there, a nova eruption arises when the accreted hydrogen rich material ignites under degenerate conditions on the surface of the white dwarf. This thermonuclear runaway (TNR) heats the envelope of the white dwarf to temperatures $\geq 3 \times 10^{5} \mathrm{~K}$.

Observationally, most post novae are characterised by - apparently - rather high accretion rates, and a slow decline of the visual brightness has been observed in a large number of systems for many years after the nova explosion (Vogt 1990; Duerbeck 1992). Clearly, CVs and novae must be closely interrelated, but the high accretion rate in post novae poses two important hurdles for our understanding of this relation (e.g. Bode \& Evans 1989, and references therein). (a) The space density of bright post novae implied by the observed rate of classical novae is much higher than the total space density of all known CVs. (b) With accretion rates as high as derived from the observations of post novae, the envelope of the accreting white dwarf in these systems can not reach the degenerate condition necessary for producing again a powerful explosive nova explosion. Both issues 
can be solved if the accretion rate in post novae drops significantly at some point after the nova explosion.

Such a decline of the mass transfer rate in post novae is the hallmark of the "hibernation scenario", which was invoked by Shara et al. (1986, see Shara 1989 for a review) to resolve the discrepancies. In the hibernation scenario, irradiation of the secondary star by the hot white dwarf should keep the mass transfer rate high after the nova eruption for a limited period of time, after which the mass transfer rate decreases to very low values. The post novae should, hence, appear at first as a nova-like system (for many decades to a century) with stable hot accretion discs, and evolve thereafter into inconspicuous low $\dot{M} \mathrm{CV}$. Thus, in the hibernation scenario it appears plausible that most "fresh" post novae have indeed high accretion rates, and that the oldest recovered novae are intrinsically very faint (e.g Shara et al. 1985).

Observational support for the hibernation scenario was claimed to come from the visual fading of many post novae, being interpreted as a slow decrease of the mass transfer rate resulting from decreasing irradiation of the secondary.

In the following section I show that an important omission in this interpretation is that so far mass transfer rates for post nova systems were derived under the assumption that the disc luminosity is due to viscous dissipation only. I showed that the nova event has important implications for the accretion disc as the slowly cooling white dwarf is intensively irradiating the accretion disc. In section 7.3.3 I demonstrated that irradiation from the hot white dwarf prevents the occurrence of dwarf nova outbursts in post nova accretion discs for up to $\sim 100 \mathrm{yrs}$. Here I show that the decline of the visual brightness observed in post novae is most likely the result of the decreasing irradiation from the white dwarf and is not related to a decrease of the mass transfer rate.

\subsection{The visual decline in post novae and disc irradi- ation}

The long term simulation of post novae (Figs. 7.10 and 7.11) shows that the decreasing disc irradiation by the cooling white dwarf results in a slow decrease of the optical brightness. Here I derive a simple estimate of the visual decline for the comparison with the observations. Smak (1989) calculated the absolute magnitudes $M_{V}$ of accretion discs as a function of the accretion rate, the mass of the central star, and the radius of the disc. At high accretion rates, $\dot{M}_{\text {acc }} \geq 10^{17} \mathrm{~g} \mathrm{~s}^{-1}$, 


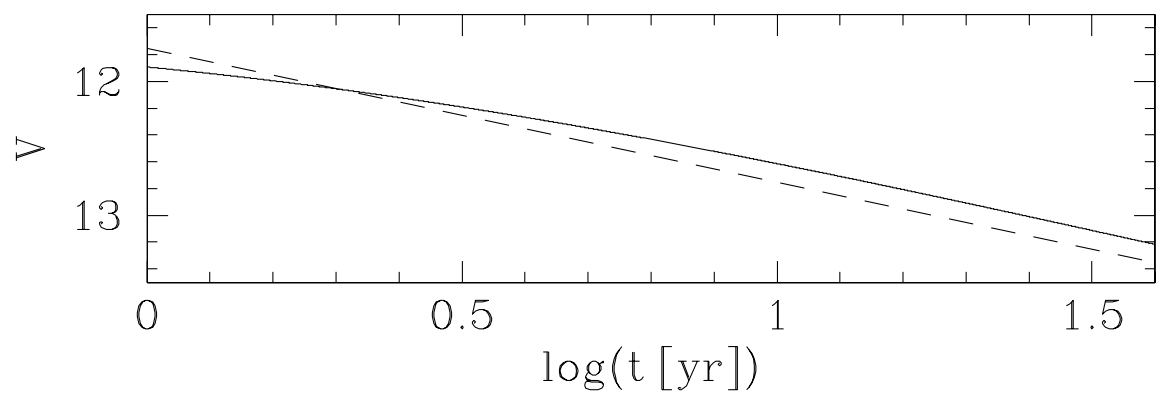

Figure 9.1: The visual decline in post novae resulting from disc irradiation in a logarithmic scale. The solid line results from the detailed calculations (see Fig. 7.10(b)) and the dashed line represents the analytic expression given in Eq. (9.5).

he finds:

$$
\frac{d M_{V}}{d \log \dot{M}_{\mathrm{acc}}} \simeq-1
$$

Using Eq.(7.21) I derive for $R \gg R_{\mathrm{wd}}$ :

$$
T_{\mathrm{irr}} \propto R^{-3 / 4}
$$

(see also King 1997). The viscous heating of the discs photosphere $T_{\mathrm{vis}}$ in a steady thin accretion disc has the same radial dependence:

$$
T_{\mathrm{vis}} \propto R^{-3 / 4} \propto \dot{M}_{\mathrm{acc}}^{1 / 4}
$$

Therefore I can estimate the decline of the visual brightness due to the decreasing effect of disc irradiation by the (cooling) hot white dwarf replacing the accretion rate with the luminosity of the white dwarf (note: $T_{\text {vis }}^{4} \propto \dot{M}, T_{\text {irr }}^{4} \propto L_{\mathrm{wd}}$ ) in Smaks relation, Eq. (9.1). The time-dependence of the white dwarf's luminosity is given by Eq. (7.22)

$$
\frac{d \log L_{\mathrm{wd}}}{d \log t}=-1
$$

and I obtain:

$$
\frac{d M_{V}}{d \log t} \simeq 1
$$

This simple estimate is in excellent agreement with the visual decline computed from the detailed light curve simulations discussed in section 7.3.4 (Fig. 7.10). It appears unavoidable that the decreasing irradiation from the slowly cooling white dwarf will result in an observable decline of the visual brightness over many years after the nova explosion. Indeed, the data compiled by Duerbeck (1992) for 13 
post novae, covering observations obtained 24 to 101 years after their nova explosions, give a mean decline rate of

$$
\frac{d M_{V}}{d \log t}=1.0 \pm 0.3
$$

which is entirely consistent with the prediction.

Smaks relation - Eq. (9.1) - is only valid for high accretion rates (i.e. greater than $\sim 10^{17} \mathrm{~g} \mathrm{~s}^{-1}$ depending somewhat on the mass of the primary and the disc radius). For low $\dot{M}$ he finds $d M_{V} / \log \left(\dot{M}_{\text {acc }}\right) \simeq-2$. In the estimation Eqs. (9.19.5) this would lead to a steeper decline for less luminous white dwarfs (notice, I replaced the accretion rate by the luminosity of the white dwarf) but for the hot white dwarfs in post novae Eq. (9.5) is more accurate.

\subsection{Mass transfer cycles instead of hibernation?}

In the hibernation model reviewed in section 9.2.1, irradiation of the secondary star by the hot white dwarf results in an enhanced mass transfer in "young" post novae by up to two orders of magnitude (Kovetz et al. 1988). The decreasing irradiation by the cooling white dwarf should then lead to a decrease of the mass transfer,

$$
\frac{d \log \dot{M}_{\mathrm{acc}}}{d \log t}=-0.45
$$

Duerbeck (1992) used this result and $d M_{\mathrm{V}} / d \log \dot{M}_{\mathrm{acc}} \simeq-1.56$ to obtain a visual decline rate of

$$
d M_{\mathrm{V}} / d \log t=0.7
$$

which was consistent with his data, Eq. (9.6), and which was thought to be an observational support of the hibernation scenario. However, the detailed simulations as well as the simple estimate above show that the observed decline of the visual brightness of post novae can be explained by the decreasing disc irradiation from the cooling white dwarf alone.

As Kovetz et al. (1988) did not consider the shielding effect of the $L_{1}$-point of the secondary by the accretion disc I advocate caution in accepting irradiated secondaries as the cause for high mass transfer rates. It is so far not clear if an irradiation-induced increase of the mass transfer still works in the presence of an accretion disc which would surely and even a few days after eruption shield the $L_{1}$-point (Leibowitz et al. 1992). The only way to obtain irradiation-induced mass transfer on a short timescale, even in the case of disc shielding, is to assume meridional circulations which transport energy from the illuminated regions to 
the $L_{1}$-region (Sarna 1990; Kirbiyik \& Smith 1976; Kippenhahn \& Thomas 1979; Meyer \& Meyer-Hofmeister 1983). The process of meridional circulations is not well understood and, hence, at least the level by which mass transfer might be increased is very uncertain.

It is also interesting to note that even though the decreasing irradiation from the white dwarf permits thermal disc instabilities a few decades after the nova explosion, most old post novae do not show dwarf nova outbursts. As pointed out at the beginning of section 7.3 V446 Her is the only known CV with a typical orbital period which shows regular dwarf nova outbursts. The conclusion from this census must be the following: in most post novae the accretion rate is too high to permit dwarf nova outbursts.

The generally high mass transfer rates of post novae, combined with the fact that the observed visual decline is more likely to result from the decreasing disc irradiation than from a decrease in the mass transfer rate, it becomes necessary to explore alternatives to the hibernation scenario.

One possibility is to assume long-term mass transfer cycles which would provide a solution because (a) a nova eruption occurs more likely when the mass transfer is high as the accreted envelope of the white dwarf has to reach a critical mass $M_{\text {env }}=1.7 \times 10^{4} R_{9}^{2.8} M_{\mathrm{wd}}^{0.7} \dot{M}_{\odot}($ Warner 1995$)$ to ignite, and (b) periods in which the $\mathrm{CVs}$ remain at very low mass transfer rates allow strong TNR for mass transfer rates $>10^{-8} M_{\odot} \mathrm{yr}^{-1}$ because the accreted matter has had enough time to degenerate. A natural cause of such long term cycles is given by King et al. (1995, 1996); McCormick \& Frank (1998) and Ritter et al. (2000) who calculated a limit-cycle behaviour of the mass transfer rate operating on the thermal timescale of the secondary ( $\sim 10^{5}$ years). The base of this limit-cycle model results from the fact, that the long term behaviour of irradiated star's is not very sensitive to how (or if) energy is deposited in its outer layers (Podsiadlowski 1991) but that the (small) part which penetrates the photosphere blocks a portion of the stars luminosity by suppressing convection which causes the star to swell. Increased mass transfer then leads to increased irradiation until the Roche lobe increases faster than the star (King et al. 1995).

The resulting long term cycles provide an excellent explanation for both the observed high mass transfer rates in post novae as well as for the dispersion of mass transfer rates in CVs for a given orbital period (see section 2.4) - two central problems of the $\mathrm{CV}$ evolution. Therefore it seems very promising to derive mass transfer rates of post novae from observations considering the effects of disc irradiation in order to compare the results with theoretically obtained transfer rates taking into account the mass transfer cycles above. 


\section{Chapter 10}

\section{Summary}

In this thesis I examined the influence that external conditions, namely mass transfer variations, stream overflow and irradiation have on accretion discs around white dwarfs in CVs.

From the discussions and conclusions presented in chapter 5 and chapter 6 it becomes clear that the conditions associated with the addition of mass to the accretion disc (i.e. the variations in the mass transfer rate and the form of mass deposition) affect the resulting light curve but do not change the overall outburst behaviour:

- Real mass transfer rates, like those derived from long term monitoring of the magnetic CV AM Her can have strong influence on the morphology of the outburst light curve. I found that even during long periods of low transfer rates the disc produces at least small outbursts. This is due to the fact that only a small fraction of the disc mass is accreted by the white dwarf during each small outburst. Even during long, large outbursts only one third of the disc mass is accreted onto the white dwarf. The remaining mass can account for many small outbursts as these reduce the mass of the accretion disc only by $\sim 1-5 \%$. Therefore, the sudden low states of VY Scl stars cannot result from low mass transfer rates alone. It is also interesting to note that the mass accretion rate of the disc relaxes to an equilibrium with the prevailing mass transfer rate on a rather short timescale. Hence, I conclude that the changes in outburst duration and outburst magnitude observed in most dwarf novae are caused by variations of the mass loss rate from the secondary. It might be a good idea to use the observed long term light curve of dwarf novae to derive the evolution of the mass transfer rate in these systems. For systems with short orbital periods and hence a short relaxation timescale (see Eq. (5.6)) the variations are expected to be very similar to 
those in magnetic systems.

- Stream overflow can cause the reflection of the inward moving heating wave when the amount of stream overflow exceeds $25 \%$. Then the stream can reverse an inward travelling cooling front and create an outward-travelling heating front because the overflowing stream material is added directly into the cooling front. For realistic amounts of stream overflow the overall outburst behaviour hardly changes. Therefore the thermal limit cycle model is in agreement with SPH-simulations of the stream disc impact (e.g Armitage \& Livio 1996).

In contrast to the mass transfer effects, the structure of the accretion disc is dramatically changed by irradiation from the accreting white dwarf. I showed that efficient irradiation causes "echo" outbursts following the major outbursts which contrasts with the observed light curves of dwarf novae. The prediction of "echo" outbursts does not depend on the assumed viscosity. I suggested that this qualitative discrepancy between observed and calculated light curves can be solved by assuming a large value for the disc albedo $\beta$, i.e. a low reprocessing efficiency. This suggestion is in agreement with results that I obtained from detailed simulations of irradiated discs in post novae. These systems provide a supreme environment for studying the effects of disc irradiation, as the white dwarf heated during the nova eruption irradiates the disc much stronger than it is the case in normal dwarf novae. In addition, the irradiation is time-dependent and, hence, during its evolution a post nova disc goes through stages with different amounts of disc irradiation.

Analysing the effects of disc irradiation in post novae I obtained the following results:

- Irradiation by the cooling white dwarf suppresses dwarf nova outbursts for up to $\sim 100 \mathrm{yr}$ depending on the orbital period (i.e. the outer disc radius), the temperature of the white dwarf after the hydrogen burning turned off and the assumed reprocessing efficiency of the accretion disc.

- The photometric history of the single known post nova which shows regular dwarf nova outbursts and has a typical CV orbital period, i.e. V446 Her, can be quantitatively reproduced by the simulations.

- The outburst frequency decreases with decreasing white dwarf temperature because larger parts of the disc are involved in the outbursts.

- The magnitude of the outbursts in post nova discs increases with time because during quiescence the brightness of the disc decreases with decreasing irradiation. 
- In agreement with a low reprocessing efficiency implied by the absence of "echo" outbursts in normal dwarf nova systems, the observations of V $446 \mathrm{Her}$ suggest a low value for $(1-\beta)$.

As mentioned in the conclusion of chapter 7 the overall observational situation is in qualitative agreement with my results. Nevertheless, it is rather unsatisfactory that no "echo" outbursts have ever been observed in CVs. Hence, the model for the dynamical behaviour of irradiated accretion discs developed here is not based on a firm observational ground yet. In order to improve this situation I have started an intensive observing campaign to obtain long term monitoring of V446 Her. The preliminary results presented in chapter 8 indeed indicate the presence of "echo" outbursts in the light curve of V446 Her. Further monitoring together with knowing the temperature of the white dwarf (derived from the proposed - and hopefully allocated - HST observations) will make it possible to place strong constraints on the most important parameter in the calculations of irradiated discs - the disc albedo $\beta$.

The presented research does not only improve our knowledge of the physics of accretion discs but also provides strong support for a new post nova scenario. In chapter 9 I showed that decreasing disc irradiation provides a self-consistent explanation for the observed visual decline in post novae. Therefore I suggest a scenario in which mass transfer cycles account for nova-active and nova-inactive stages. Such a scenario solves the problem of the dispersion of the mass transfer rates for a given orbital period (see section 2.4) as well as it explains the rather high mass transfer rates in post nova systems. 


\section{Bibliography}

Abramowicz M.A., Czerny B., Lasota J.P., Szuszkiewicz E., 1988, ApJ 332, 646

Abramowicz M.A., Chen X., Granath M., Lasota J.P., 1996, ApJ 471, 762

Adams F.C., Shu F.H., Lada C.J., 1988, ApJ 326, 865

Armitage P.J., Livio M., 1996, ApJ 470, 1024

Armitage P.J., Livio M., 1998, ApJ 493, 898

Barrett P., O’Donoghue D., Warner B., 1989, MNRAS 236, 735

Bath G.T., Pringle J.E., 1981, MNRAS 194, 967

Bath G.T., Edwards A.C., Mantle V.J., 1983, in IAU Colloq. 72: Cataclysmic Variables and Related Objects, pp. 55-66

Beckwith S.V.W., 1994, in Theory of Accretion Disks - 2, p. 1

Begelman M.C., 1978, MNRAS 184, 53

Beuermann K., Burwitz V., 1995, in Buckley \& Warner (1995), p. 99

Bisnovatyi-Kogan G.S., Lovelace R.V.E., 2000, ApJ 529, 978

Blandford R.D., 1992, in Physics of Active Galactic Nuclei, p. 3

Blandford R.D., Begelman M.C., 1999, MNRAS 303, L1

Bode M.F., Evans A., 1989, Classical novae (Chichester: Wiley, 1989, edited by Bode, M.F.; Evans, A.)

Bondi H., 1952, MNRAS 112, 195

Buckley D.A.H., Warner B. (eds.), 1995, Cape Workshop on Magnetic Cataclysmic Variables (ASP Conf. Ser. 85) 
Burderi L., King A.R., Szuszkiewicz E., 1998, ApJ 509, 85

Cannizzo J.K., 1993a, ApJ 419, 318

Cannizzo J.K., 1993b, in Accretion disks in compact stellar objects, Wheeler J. (ed.), Advanced Series in Astrophysics and Cosmology 9, pp. 6-40 (Singapore: World Scientific)

Cannizzo J.K., Cameron A.G.W., 1988, ApJ 330, 327

Cannizzo J.K., Mattei J.A., 1992, ApJ 401, 642

Cannizzo J.K., Wheeler J.C., 1984, ApJ 55, 367

Cannizzo J.K., Wheeler J.C., Polidan R.S., 1986, ApJ 301, 634

Cropper M., Ramsay G., Wu K., 1998, MNRAS 293, 222

Cropper M., Wu K., Ramsay G., Kocabiyik A., 1999, MNRAS 306, 684

de Kool M., 1992, A\&A 261, 188

Dgani R., Livio M., 1984, MNRAS 210, 393

Downes R.A., Shara M.M., 1993, PASP 105, 127

Dubus G., Lasota J., Hameury J., Charles P., 1999, MNRAS 303, 139

Duerbeck H.W., 1987, Space Science Reviews 45, 1

Duerbeck H.W., 1992, MNRAS 258, 629

Duschl W.J., Livio M., 1989, A\&A 209, 183

Eggleton P.P., 1983, ApJ 268, 368

Frank J., King A.R., Raine D.J., 1992, Accretion power in astrophysics (Cambridge: Cambridge University Press)

Gänsicke B.T., Beuermann K., de Martino D., 1995, A\&A 303, 127

Gänsicke B.T., Hoard D.W., Beuermann K., Sion E.M., Szkody P., 1998a, A\&A 338,933

Gänsicke B.T., van Teeseling A., Beuermann K., de Martino D., 1998b, A\&A 333,163 
Gaposchkin C.H.P., 1957, The galactic novae. (Amsterdam, North-Holland Pub.

Co.; New York, Interscience Publishers, 1957.)

Genzel R., Eckart A., Ott T., Eisenhauer F., 1997, MNRAS 291, 219

Giannone P., Weigert H., 1967, Z. Astroph. 67, 41

Gonzalez-Riestra R., Orio M., Gallagher J., 1998, A\&AS 129, 23

Greeley B.W., Blair W.P., Long K.S., Raymond J.C., 1999, ApJ 513, 491

Guilbert P.W., Rees M.J., 1988, MNRAS 233, 475

Hamada T., Salpeter E.E., 1961, ApJ 134, 683

Hameury J., Menou K., Dubus G., Lasota J., Hure J., 1998, MNRAS 298, 1048

Hameury J.M., Lasota J.P., Dubus G., 1999, MNRAS 303, 39

Hameury J.M., Lasota J.P., Warner B., 2000, A\&A 353, 244

Harrison T.E., Gehrz R.D., 1988, AJ 96, 1001

Harrison T.E., Gehrz R.D., 1991, AJ 101, 587

Hessman F.V., 1987, Ap\&SS 130, 351

Hessman F.V., 1999, ApJ 510, 867

Hessman F.V., Gänsicke B.T., Mattei J.A., 2000, A\&A 361, 952

Hjellming M.S., Taam R.E., 1991, ApJ 370, 709

Honeycutt R.K., Robertson J.W., Turner G.W., 1998, AJ 115, 2527

Ichikawa S., Osaki Y., 1992, PASJ 44, 15

Ichimaru S., 1977, ApJ 214, 840

Kato S., Fukue J., Mineshige S. (eds.), 1998, Black-hole accretion disks

Katz J.I., 1977, ApJ 215, 265

King A.R., 1997, MNRAS 288, L16

King A.R., 1998, MNRAS 296, L45

King A.R., Cannizzo J.K., 1998, ApJ 499, 348 
King A.R., Whitehurst R., Frank J., 1990 244, 731

King A.R., Frank J., Kolb U., Ritter H., 1995, ApJ Lett. 444, L37

King A.R., Frank J., Kolb U., Ritter H., 1996, ApJ 467, 761

Kippenhahn R., 1967, in Proceedings of the Colloquium, held in Trieste, June 13-17, 1966, Trieste: Osservatorio Astronomico, 1967, edited by Hack, Margherita, p.319, p. 319

Kippenhahn R., Thomas H.., 1979, A\&A 75, 281

Kirbiyik H., Smith R.C., 1976, MNRAS 176, 103

Kolb U., Ritter H., 1992, A\&A 254, 213

Kolb U., Stehle R., 1996, MNRAS 282, 1454

Kovetz A., Prialnik D., Shara M.M., 1988, ApJ 325, 828

Krautter J., Ögelman H., Starrfield S., Wichmann R., Pfeffermann E., 1996, ApJ 456, 788

Leach R., Hessman F.V., King A.R., Stehle R., Mattei J., 1999, MNRAS 305, 225

Leibowitz E.M., Mendelson H., Mashal E., Prialnik D., Seitter W.C., 1992, ApJ Lett. 385, L49

Lightman A.P., Eardley D.M., 1974, ApJ Lett. 187, L1

Livio M., Pringle J.E., 1992 259, 23P

Livio M., Pringle J.E., 1994, ApJ 427, 956

Livio M., Soker N., Dgani R., 1986, ApJ 305, 267

Long K.S., Mauche C.W., Raymond J.C., Szkody P., Mattei J.A., 1996, ApJ 469, 841

Lubow S.H., 1989, ApJ 340, 1064

Lubow S.H., Shu F.H., 1975, ApJ 198, 383

Lubow S.H., Shu F.H., 1976, ApJ Lett. 207, L53

Ludwig K., Meyer F., 1998, A\&A 329, 559

Ludwig K., Meyer-Hofmeister E., Ritter H., 1994, A\&A 290, 473 
Lynden-Bell D., 1969, Nat 223, 690

Lynden-Bell D., Pringle J.E., 1974, MNRAS 168, 603

MacDonald J., 1983, ApJ 267, 732

MacDonald J., 1996, in Cataclysmic Variables and Related Objects, Evans A., Wood J.H. (eds.), IAU Coll. 158, pp. 281-287 (Dordrecht: Kluwer)

Malbet F., Rigaut F., Bertout C., Lena P., 1993, A\&A 271, L9

Marcy G.W., Butler R.P., Vogt S.S., Fischer D., Liu M.C., 1999, ApJ 520, 239

Matsumoto K., Fukue J., 1998, PASJ 50, 89

McCormick P., Frank J., 1998, ApJ 500, 923

McDermott P.N., Taam R.E., 1989, ApJ 342, 1019

Meyer F., Meyer-Hofmeister E., 1983, A\&A 121, 29

Meyer F., Meyer-Hofmeister E., 1984, A\&A 132, 143

Mineshige S., 1988, A\&A 190, 72

Mineshige S., Tuchman Y., Wheeler J.C., 1990, ApJ 359, 176

Mouchet M., 1993, in White Dwarfs: Advances in Observation and Theory, Barstow M.A. (ed.), pp. 411-417 (Dordrecht: Kluwer)

Mukai K., Charles P.A., 1987, MNRAS 226, 209

Narayan R., Yi I., 1994, ApJ Lett. 428, L13

Narayan R., Yi I., 1995, ApJ 444, 231

Osaki Y., 1989, in Theory of Accretion Disks, p. 183

Patterson J., 1984, ApJS 54, 443

Piran T., 1978, ApJ 221, 652

Podsiadlowski P., 1991, Nat 350, 136

Prialnik D., 1986, ApJ 310, 222

Pringle J.E., 1981, ARA\&A 19, 137 
Pylyser E., Savonije G.J., 1988, A\&A 191, 57

Pylyser E.H.P., Savonije G.J., 1989, A\&A 208, 52

Rees M.J., Phinney E.S., Begelman M.C., Blandford R.D., 1982, Nat 295, 17

Ritter H., 1976, MNRAS 175, 279

Ritter H., 1988, A\&A 202, 93

Ritter H., Zhang Z.., Kolb U., 2000, A\&A 360, 959

Salpeter E.E., 1964, ApJ 140, 796

Sarna M.J., 1990, A\&A 239, 163

Schenker K., Kolb U., Ritter H., 1998, MNRAS 297, 633

Sekiguchi K., 1992, Nat 358, 563

Shakura N.I., Sunyaev R.A., 1973, A\&A 24, 337

Shanley L., Ögelman H., Gallagher J.S., Orio M., Krautter J., 1995, ApJ Lett. 438, L95

Shapiro S.L., Lightman A.P., Eardley D.M., 1976, ApJ 204, 187

Shara M.M., 1989, PASP 101, 5

Shara M.M., Moffat A.F.J., Webbink R.F., 1985, ApJ 294, 271

Shara M.M., Livio M., Moffat A.F.J., Orio M., 1986, ApJ 311, 163

Shugarov S.Y., 1983, Peremennye Zvezdy 21, 807

Smak J., 1984, Acta Astron. 34, 317

Smak J., 1985, Acta Astron. 35, 351

Smak J., 1989, Acta Astron. 39, 317

Smak J., 1991, Acta Astron. 41, 269

Smak J., 1996, in IAU Colloq. 158: Cataclysmic Variables and Related Objects, p. 45

Smak J., 1998, Acta Astron. 48, 677 
Smak J., 1999, Acta Astron. 49, 383

Smith B.A., Terrile R.J., 1984, Science 226, 1421

Somers M.W., Naylor T., 1999, A\&A 352, 563

Somers M.W., Mukai K., Naylor T., 1996, MNRAS 278, 845

Starrfield S., Sparks W.M., Truran J.W., 1985, ApJ 291, 136

Starrfield S., Sparks W.M., Truran J.W., 1986, ApJ Lett. 303, L5

Starrfield S., Truran J., Sparks M., et al., 1998, in Wild Stars in the Old West: Proceedings of the 13th North American Workshop on CVs and Related Objects, Howell S., Kuulkers E., Woodward C. (eds.), pp. 352-367 (ASP Conf. Ser. 137)

Stehle R., King A.R., 1999, MNRAS 304, 698

Stienon F.M., 1971, PASP 83, 363

Suleimanov V., Meyer F., Meyer-Hofmeister E., 1999, A\&A 350, 63

Szkody P., Long K.S., Sion E.M., Raymond J.C., 1996, ApJ 469, 834

Taam R.E., Bodenheimer P., 1991, ApJ 373, 246

Thorne K.S., Price R.H., 1975, ApJ Lett. 195, L101

Thorstensen J.R., Taylor C., 2000, MNRAS 312, 629

Tuchman Y., Mineshige S., Wheeler J.C., 1990, ApJ 359, 164

van den Bergh S., Younger P.F., 1987, A\&AS 70, 125

Vogt N., 1990, ApJ 356, 609

Warner B., 1987, MNRAS 227, 23

Warner B., 1995, Cataclysmic Variable Stars (Cambridge: Cambridge University Press)

Wu K., Chanmugam G., Shaviv G., 1995, ApJ 455, 260

Young P., Schneider D.P., Shectman S.A., 1981, ApJ 245, 1043 


\section{Acknowledgement}

First of all I thank my supervisor PD Dr. Karl Mannheim for giving me the opportunity to pursue my interest in the fascinating field of accretion discs in CVs and for his helpfulness. Without his generosity and coolness, I would not have found my way during the darker periods of the last years.

I am especially grateful to my friend and colleague Dr. Boris T. Gänsicke for his burning enthusiasm in astrophysics and his hardly ending patience with all my questions concerning everything from $\mathrm{CVs}$ and astrophysics in general to computer problems. Without your support this thesis would not be the same. I also thank you very much for carefully reading the manuscript while I am typing these words.

I would also like to thank Prof. Klaus Beuermann, and especially Rick Hessman for their support during the last years.

This research would not have been possible without the financial support by the DFG (MA 1545/2-1).

A special thank is extended to Dr. John K. Cannizzo for providing me with a copy of his vertical structure code. I also thank Elena Pavlenko, Rich Williams, Gianluca Masi, Tonny Vanmunster and Lasse Teist Jensen for joining the VSNET campaign on V446 Her.

I gratefully acknowledge stimulating discussions as well as extended nonsense with Frank Rieger (the man who is dealing with the most horrible equations I have ever seen; how did he get through?), Tanja Kneiske (Prof. in preparation) and Christian Hettlage (really advanced English speaker). It was a pleasure to work with you in the same room.

I am grateful to my parents for their general support during the last 31 years.

The deepest thank is extended to my wife Katrin for making me see clearly, putting my feet back on the ground and giving me the really important things in life. Luv ya. 



\section{List of publications}

\section{Refereed Journals}

Schreiber M.R., Gänsicke B.T., Irradiated accretion discs in post novae, 2000, A\&A submitted

Schreiber M.R., Hessman F.V., Stream Overflow and dwarf nova eruptions, 1998, MNRAS 301, 626

Schreiber M.R., Gänsicke B.T., Cannizzo J., On the occurrence of dwarf nova outbursts in post novae, 2000, A\&A 362, 268

Schreiber M.R., Gänsicke B.T., Hessman F.V.,The response of a dwarf nova disc to real mass transfer rates, 2000, A\&A 358, 221

\section{Conference proceedings}

Schreiber M.R., 1999, AG Abstract Services, vol. 15. Abstracts of Contributed Talks and Posters presented at the Annual Scientific Meeting of the Astronomische Gesellschaft, in Goettingen, September 20-25, 1999 15, 12

Schreiber M.R., 1998, Astronomische Gesellschaft Meeting Abstracts, Abstracts of Contributed Talks and Posters presented at the Annual Scientific Meeting of the Astronomische Gesellschaft at Heidelberg, September 14-19, 1998 14, 39

Schreiber M.R., Hessman F.V., 1997, Astronomische Gesellschaft Meeting Abstracts, Abstracts of Contributed Talks and Posters presented at the Annual Scientific Meeting of the Astronomische Gesellschaft at Innsbruck, September 22-27, 1997 13, 153

Schreiber M.R., Hessman F.V., 1998, in ASP Conf. Ser. 137: Wild Stars in the Old West: Proceedings of the 13th North American Workshop on Cataclysmic Variables and Related Objects. ASP Conference Series, Vol.137, eds. S. Howell, E. Kuulkers, and C. Woodward (1998), p.541 



\section{Curriculum Vitae}

Name:

Born:

Nationality:

Marital status:

Sept. 1975 - July 1979:

Sept. 1979 - June 1988:

Jan. 1989 - Aug. 1990:

Oct. 1990 - Feb. 1997:
Matthias R. Schreiber

September 7, 1969 Göttingen

German

Married with Katrin Noack since August 13, 1999
Lohberg Grundschule in Göttingen

Georg Christoph Lichtenberg Gesamtschule, Göttingen/Geismar,

Acquisition of the German matriculation standard, Abitur

Community service as alternative to military service at the KEI-Kindergarten in Göttingen

Studies of physics at the Georg-August-Universität in Göttingen, diploma in physics, title of the diploma-thesis: Stromüberlauf in Kataklysmischen Variablen

March 1997 - Jan. 2001: $\quad$ PhD studies in astrophysics at the Universitäts-Sternwarte in Göttingen, supervisor: Karl Mannheim 